GEOHYDROLOGY, GROUND-WATER QUALITY, AND SIMULATED GROUND-WATER FLOW, GEAUGA COUNTY, OHIO

By Sandra M. Eberts, E. Scott Bair, and Jeffrey T. de Roche

U.S. GEOLOGICAL SURVEY

Water-Resources Investigations Report 90-4026

Prepared in cooperation with the

GEAUGA COUNTY PLANNING COMMISSION
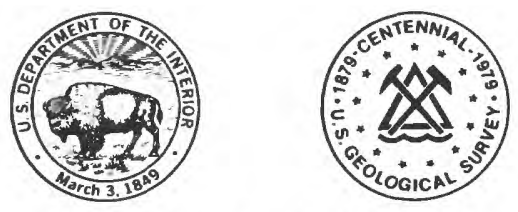

Columbus, Ohio 1990 
DEPARTMENT OF THE INTERIOR

MANUEL LUJAN, JR, Secretary

U.S. GEOLOGICAL SURVEY

Dallas L. Peck, Director

For additional information write to:

District Chief

Water Resources Division

U.S. Geological Survey

975 W. Third Avenue

Columbus, Ohio 43212-3192
Copies of this report can

be purchased from:

U.S. Geological Survey

Books and Open-File Reports

Box 25425, Federal Center

Building 810

Denver, CO 80225 


\section{CONTENTS}

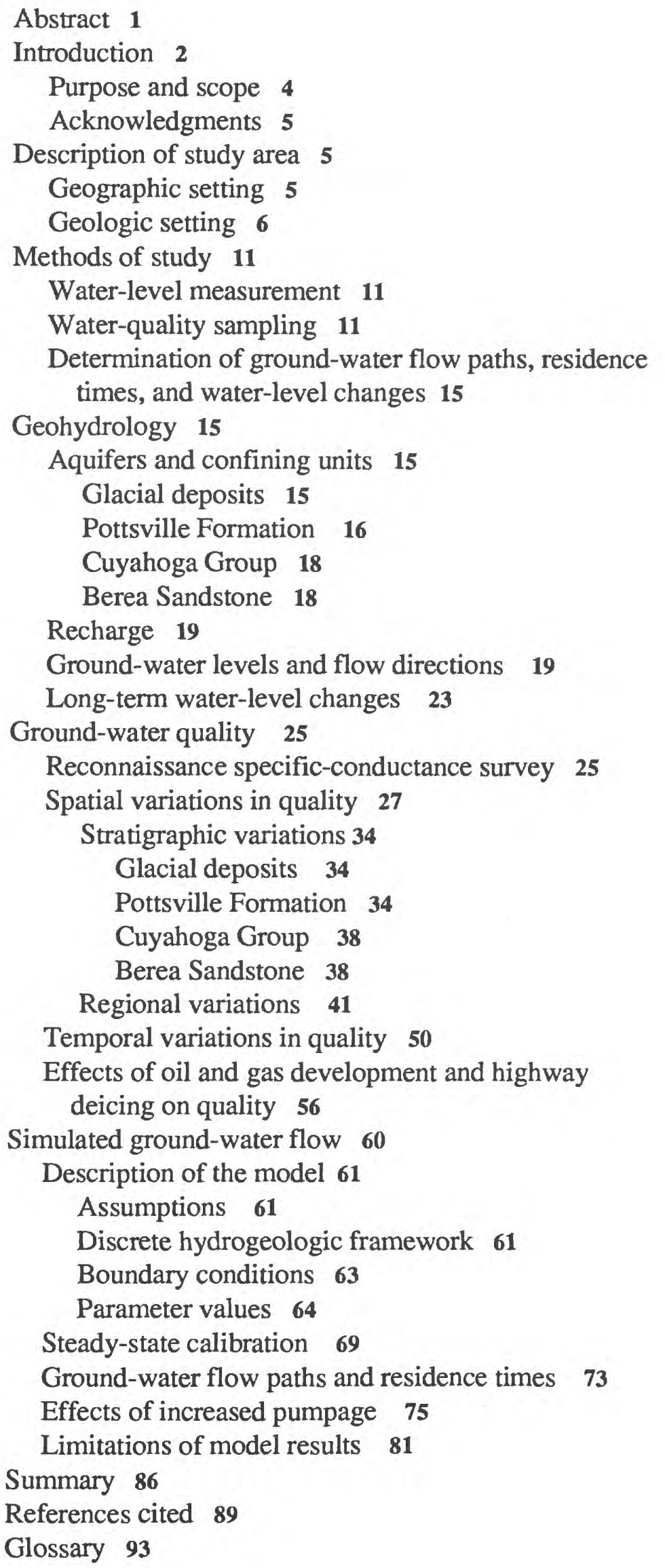




\section{ILLUSTRATIONS}

[Plates are in pocket]

Plate 1. Map showing bedrock geology, altitude of top of bedrock, and location of geologic sections

2. Generalized geologic sections of Geauga County, Ohio

Figure 1-8. Maps showing:

1. Location of Geauga County, modeled area, and major drainage basins 3

2. Average annual precipitation, 1931-80 7

3. Location of buried valleys and aquifer-test sites $\mathbf{1 0}$

4. Sites at which ground-water levels were measured 12

5. Wells sampled for water-quality analysis $\mathbf{1 4}$

6. Ground-water levels in the Pottsville Formation and the glacial deposits, January-February 198620

7. Ground-water levels in the Cuyahoga Group January-February 198621

8. Ground-water levels in the Berea Sandstone, January-February 198622

9-13. Graphs showing:

9. Long-term ground-water-level changes in the glacial deposits 24

10. Long-term ground-water-level changes in the Pottsville Formation 24

11. Long-term ground-water-level changes in the Cuyahoga Group 26

12. Long-term ground-water-level changes in the Berea Sandstone 26

13. Relation between specific conductance and total dissolved solids from 29 wells in Geauga County Ohio 28

14. Piper diagram showing variation in the chemical character of waters from the glacial deposits in Geauga County, Ohio 35

15. Piper diagram showing variation in the chemical character of waters from the Pottsville Formation in Geauga County, Ohio 37

16. Piper diagram showing variation in the chemical character of waters from the Cuyahoga Group in Geauga County, Ohio 39 


\section{ILLUSTRATIONS-Continued}

17. Piper diagram showing variation in the chemical character of waters from the Berea Sandstone in Geauga County, Ohio $\quad 40$

18. Stiff diagrams showing spatial variations in water quality relative to the potentiometric surface in the glacial deposits and the Pottsville Formation $\mathbf{4 4}$

19. Stiff diagrams showing spatial variations in water quality in the Cuyahoga Group 46

20. Stiff diagrams showing spatial variations in water quality quality relative to the potentiometric surface in the Berea Sandstone 48

21-24. Graphs showing:

21. Variability of specific conductance and total dissolved solids concentration in ground water from selected wells in Geauga County, Ohio, from May 1978 through May $1986 \mathbf{5 1}$

22. Variability of concentrations of dissolved calcium, dissolved magnesium, and dissolved sodium in ground water from selected wells in Geauga County, Ohio, from May 1978 through May 198652

23. Variability of concentrations of bicarbonate, dissolved chloride, and dissolved sulfate in ground water from selected wells in Geauga County, Ohio, from May 1978 through May $1986 \mathbf{5 3}$

24. Variability of concentrations of dissolved iron, total organic carbon, and dissolved ammonia in ground water from selected wells in Geauga County, Ohio, from May 1978 through May 198654

25. Bromide:chloride ratios for local ground waters and brine-mixing zones 59

26-30. Maps showing:

26. Boundaries used to simulate the ground-water-flow system represented by (A) layer 1, (B) layer 2, and (C) layer 362

27. Horizontal hydraulic conductivity values used to simulate the ground-water-flow system represented by (A) layer 1 , (B) layer 2, and (C) layer 365

28. Spatial distribution of recharge used to simulate the groundwater-flow system $\mathbf{7 0}$

29. Simulated ground-water flow paths and residence times, and the relation of simulated contours in layer 1 to ground-water levels measured in January-February $1986 \mathbf{7 1}$ 


\section{ILLUSTRATIONS-Continued}

30. Simulated ground-water flow paths and residence times, and the relation of simulated contours in layer 3 to ground-water levels measured in January-February $1986 \mathbf{7 2}$

31. General relation between ground-water flow regimes in the aquifers and the leaky regional confining unit $\mathbf{7 6}$

32-37. Maps showing:

32. Amount of drawdown that may occur if the 1985 population of Geauga County increases 12 percent to an expected population of 90,000 in $1995 \quad 79$

33. Pumpage data used to simulate the effects of a 12-percent increase in population $\mathbf{8 0}$

34. Amount of drawdown that may occur if the 1985 population of Geauga County increases 17 percent to an expected population of 94,100 , in $2000 \quad 82$

35. Pumpage data used to simulate the effects of a 17-percent increase in population $\mathbf{8 3}$

36. Amount of drawdown that may occur if the 1985 population of Geauga County increases 21 percent to an expected population of 97,200 , in 200584

37. Pumpage data used to simulate the effects of a 21-percent increase in population 85

\section{TABLES}

Table 1. Summary of rock units and associated water-bearing properties in Geauga County, Ohio 8

2. Records of selected wells in Geauga, Lake, Ashtabula, Trumbull, and Portage Counties, Ohio (Back of report)

3. Listing of concentrations of chemical constituents and physical properties of waters from the glacial deposits, Pottsville Formation, Cuyahoga Group, Berea Sandstone, and Bedford Shale in Geauga County, Ohio 30

4. Minimum, maximum, mean, and median total dissolved solids and total hardness for waters in each hydrostratigraphic unit $\mathbf{3 6}$

5. Listing of concentrations of chemical constituents and physical properties of brines from the Newburg zonein Summit County and the Clinton zone in Geauga County, and from selected residential wells in Geauga County, Ohio $\mathbf{5 8}$

6. Simulated ground-water budget from the calibrated steadystate ground-water-flow model $\mathbf{7 3}$ 


\section{CONVERSION FACTORS AND ABBREVIATIONS}

\begin{tabular}{|c|c|c|}
\hline Multiply & By & To obtain \\
\hline inch (in.) & 25.4 & millimeter (mm) \\
\hline foot (ft) & 0.3048 & meter $(\mathrm{m})$ \\
\hline $\begin{array}{l}\text { foot per day } \\
\text { (ft/d) }\end{array}$ & 0.3048 & $\begin{array}{l}\text { meter per day } \\
(\mathrm{m} / \mathrm{d})\end{array}$ \\
\hline mile (mi) & 1.609 & kilometer $(\mathrm{km})$ \\
\hline $\begin{array}{l}\text { foot squared per } \\
\text { day (ft /d) }\end{array}$ & 0.09290 & $\begin{array}{l}\text { square meter per } \\
\text { day }(\mathrm{m} / \mathrm{d})\end{array}$ \\
\hline $\begin{array}{l}\text { square mile } \\
\text { (mi ) }\end{array}$ & 2.590 & $\begin{array}{l}\text { square kilometer } \\
(\mathrm{km})\end{array}$ \\
\hline gallon (gal) & 3.785 & liter (L) \\
\hline $\begin{array}{l}\text { gallon per minute } \\
(\mathrm{gal} / \mathrm{min})\end{array}$ & 0.06308 & $\begin{array}{l}\text { liter per second } \\
(\mathrm{L} / \mathrm{s})\end{array}$ \\
\hline $\begin{array}{l}\text { gallon per day } \\
(\mathrm{gal} / \mathrm{d})\end{array}$ & 0.003785 & $\begin{array}{l}\text { cubic meter per } \\
\text { day }(\mathrm{m} / \mathrm{d})\end{array}$ \\
\hline $\begin{array}{l}\text { million gallons } \\
\text { per day (Mgal/d) }\end{array}$ & 0.04381 & $\begin{array}{r}\text { cubic meter per } \\
\text { second }(\mathrm{m} / \mathrm{s})\end{array}$ \\
\hline
\end{tabular}

Sea level: In this report "sea level" refers to the National Geodetic Vertical Datum of 1929 (NGVD of 1929) - a geodetic datum derived from a general adjustment of the firstorder level nets of both the United States and Canada, formerly called "Sea Level Datum of 1929." 


\title{
GEOHYDROLOGY, GROUND-WATER QUALITY, AND SIMULATED GROUND-WATER FLOW, GEAUGA COUNTY, OHIO
}

\author{
By Sandra M. Eberts, E. Scott Bair, and Jeffrey T. de Roche
}

\begin{abstract}
Data were collected in $1978,1980,1985$, and 1986 to assess spatial and temporal variations in ground-water quality, determine regional ground-water-flow patterns, and predict regional changes in ground-water levels that might occur with predicted increases in ground-water development in Geauga County, Ohio. The hydrogeologic setting comprises a sequence of nearly flat-lying, indurated sedimentary rocks overlain by unconsolidated glacial deposits of variable thickness. Variations in ground-water quality and ground-water-flow patterns in the County were characterized from water samples and water-level measurements from wells completed in the glacial deposits, Pottsville Formation, Cuyahoga Group, Berea Sandstone, and two oil- and gas-producing horizons.

The chemical character of ground waters in the County is stratigraphically and areally variable. Most of the waters in the glacial deposits and the Pottsville Formation, which serve as surficial aquifers, are calcium bicarbonate type waters, whereas most of the waters in the underlying Cuyahoga Group are sodium bicarbonate-type waters. Waters in the Berea Sandstone have a mixed cation-bicarbonate character. The change in cation character from calcium to sodium occurs in the Cuyahoga Group, which is composed predominantly of interbedded shales and sandstones. Cation exchange of calcium ions for sodium ions in the shales of the Cuyahoga Group is believed to be responsible for the change in cation character. The median calcium:sodium ratio of waters from the glacial deposits and the Pottsville Formation is 10.2 , as compared with 0.15 for the Cuyahoga Group. The natural water-softening process also causes an increase in total dissolved solids concentration and a decrease in total hardness of waters in the Cuyahoga Group relative to waters in the glacial deposits and the Pottsville Formation.
\end{abstract}

Regional variations in ground-water quality within a specific hydrostratigraphic unit are most common in the Cuyahoga Group and the Berea Sandstone where the open intervals of wells are directly overlain by permeable glacial deposits. These overlying deposits appear to supply water by means of vertical leakage to wells when pumped, and impart a calcium or mixed-cation character to well waters instead of the normal sodium character found in these units.

No appreciable changes in major and minor ion concentrations were detected in the ground water from 1978 through 1986, except at isolated locations where water from 
several wells had elevated concentrations of sodium, calcium, bromide, and (or) chloride due to contamination by road salts and (or) oilfield and gasfield brine. Bromide/chloride mixing curves were used to differentiate between these two origins of salinity in local waters.

A three-dimensional, steady-state flow model of the ground-water-flow system in Geauga County was constructed and used to simulate flow paths and to compute groundwater residence times. The model also was used to estimate ground-water-level declines resulting from forecasted changes in population distribution within the County and concomitant changes in ground-water pumpage. The model was calibrated against water levels measured in January-February 1986 and against data obtained from an October 1980 stream gain/loss study.

The simulated steady-state ground-water budget for Geauga County and vicinity indicates that precipitation accounts for 100 percent of recharge to the ground-water-flow system. Approximately 69 percent of ground-water discharge is to streams and lakes, 29 percent is flow across aquifer boundaries, and 2 percent is from pumping of municipal wells.

Model results indicate that flow in the glacial deposits and Pottsville Formation is from local recharge areas to immediately adjacent local discharge areas. Flow in the Cuyahoga Group is predominantly vertical. Consequently, on a regional scale, the Cuyahoga Group serves as a leaky confining unit between the overlying surficial aquifers and the underlying Berea Sandstone. Flow in the Berea Sandstone is radial in pattern and regional in character. Simulated ground-water residence times that correspond to the simulated flow paths chosen to illustrate flow within the aquifers and the regional confining unit range from 15 to 4,800 years.

Estimates of ground-water-level declines resulting from increased domestic pumpage in the surficial aquifers are based on forecasted population increases of 12,17, and 21 percent during the periods $1985-1995,1985-2000$, and 1985-2005, respectively. The areal distribution of increased pumpage was based on the distribution of forecasted population growth. The simulations indicate that the only areas of notable ground-waterlevel decline would be in Chester Township, South Russell Village, the southern part of Russell Township, and Bainbridge Township. A maximum of 8 feet of decline is estimated.

\section{INTRODUCTION}

Ground-water development for domestic water supplies is increasing in Geauga County, Ohio, as a result of urban expansion from Cleveland, which is located just west of the County (fig. 1). Development of ground water in areas where the geohydrology is poorly defined can degrade ground-water quality and lower water levels. To avoid such 


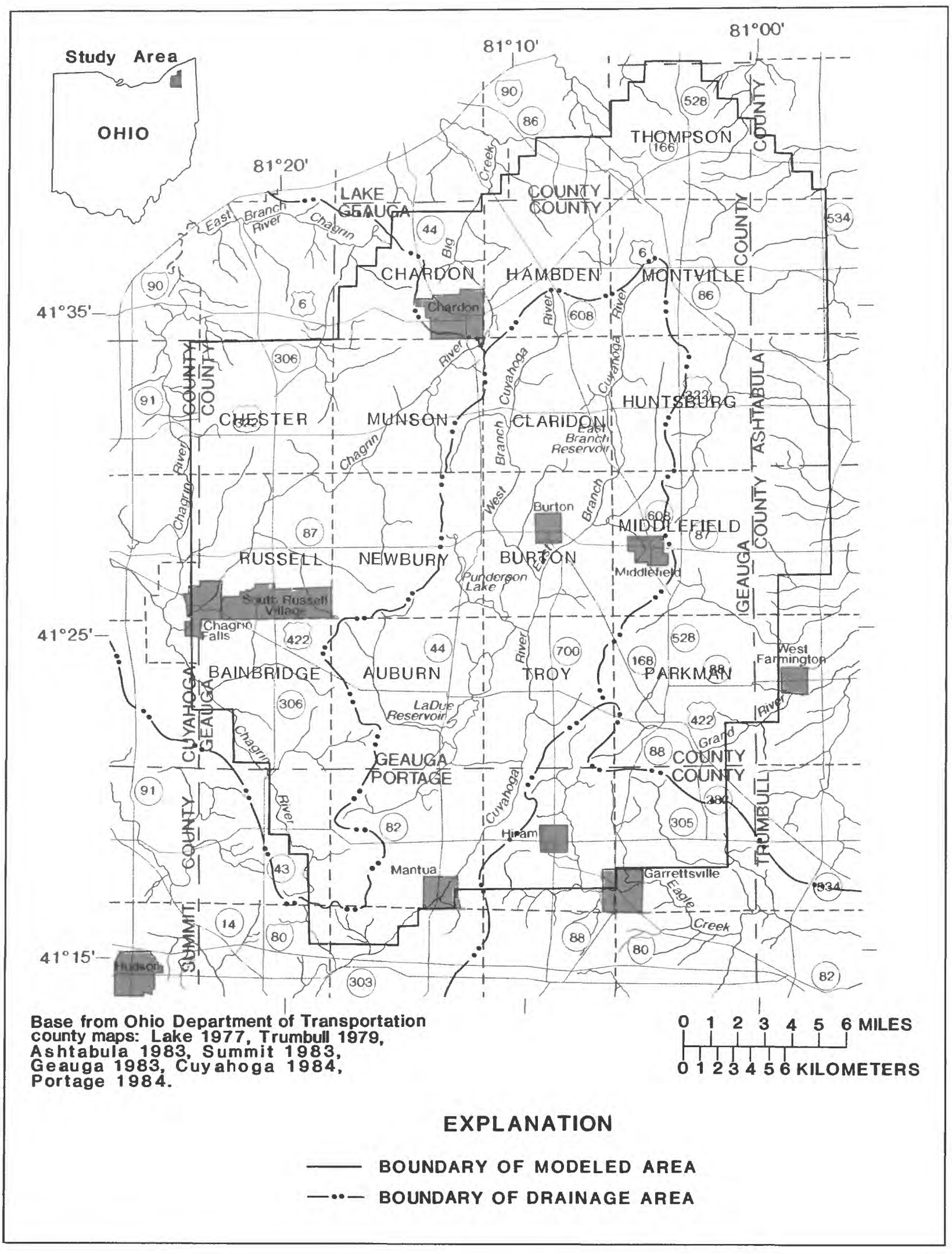

Figure 1.--Location of Geauga County, modeled area, and major drainage basins. 
negative effects on the aquifers within Geauga County, it is necessary to improve and augment the current understanding of the geohydrologic conditions within the area. In addition, the increase in oil and gas development activity in central and eastern Geauga County has created concern regarding the production and disposal of brine that usually accompanies the production of oil and gas. Brine-handling and disposal practices have the potential to contaminate potable ground-water supplies.

In 1978-1980, the U.S. Geological Survey, in cooperation with the Geauga County Sanitary Engineering Department, conducted a study that produced a generalized waterlevel map of the uppermost bedrock aquifers and defined the water quality in 12 wells and 2 springs (Nichols, 1980). On the basis of that study, a new investigation was begun in 1980 to define the ground-water-flow system in more detail, and to determine if increased ground-water withdrawal since 1978 had caused significant changes in groundwater quality and quantity. Construction of a preliminary numerical model of the ground-water flow system was started. The model was intended to aid in understanding ground-water flow within Geauga County and to predict future water-level changes due to increased usage of ground water. The project was suspended by the cooperator in 1981 because of budgetary considerations.

In 1985, the U.S. Geological Survey began an investigation in cooperation with the Geauga County Planning Commission to continue previous work to define the geohydrology of the ground-water-flow system and to determine flow directions within the County. Water levels and water-quality changes since 1978 were to be determined. The possibility of ground-water contamination from oil and gas production was to be investigated. In addition, changes likely to occur within the surficial aquifers as a result of increased ground-water development were to be predicted. This study was undertaken to contribute to the understanding of how geohydrologic information can be used by planners to avoid negative impacts on potable ground-water supplies.

\section{Purpose and Scope}

This report presents the results of the study conducted from July 1985 through October 1988. Specifically, the report identifies and characterizes the ground-water-flow regime and water quality in the aquifers and the leaky regional confining unit within the study area. Water-level maps and generalized flow directions in the aquifers are presented, as are graphs showing long-term water-level changes. Background water quality in the aquifers and the regional confining unit is documented, and long-term waterquality changes are noted. The effects of oil and gas development and highway deicing on ground-water quality within the County are discussed. Also discussed are simulated ground-water residence times and simulated effects of increased pumpage on groundwater levels in the surficial aquifers. 
The data-collection phase of this project was from August 1985 through November 1986. Data collected during the previous investigations also were utilized. The McDonald and Harbaugh (1984) three-dimensional, ground-water-flow program was used to simulate the ground-water-flow system in Geauga County. Results from the calibrated, steady-state model were used in conjunction with the particle-tracking program MODPATH (Pollock, 1989) to compute ground-water flow paths and ground-water residence times within the flow system. In addition, the calibrated model was used to predict the steady-state response of the flow system to anticipated increases in groundwater development.

\section{Acknowledgments}

The authors express thanks to members of the Geauga County Planning Commission for their assistance and to the individual well owners and oil and gas production companies who allowed their wells to be measured and (or) sampled.

\section{DESCRIPTION OF STUDY AREA}

\section{Geographic Setting}

Geauga County encompasses approximately $409 \mathrm{mi}^{2}$ (square miles) and is located in northeastern Ohio (fig. 1). The population of Geauga County in 1980 was 74,474 (U.S. Bureau of the Census, 1983), which represents an 18.3 percent increase in population from 1970. During the same period, the population of Ohio increased by 1.3 percent, whereas the population of the United States increased by 11.5 percent (U.S. Bureau of the Census, 1983). It is estimated that the population of Geauga County will grow 14 percent between 1980 and 1990 and 10.5 percent between 1990 and 2000 (Northeast Ohio Area Coordinating Agency, 1983). Chester Township, Bainbridge Township, and South Russell Village (fig. 1) are the areas within Geauga County where the greatest amount of growth is anticipated.

A recent industrial directory for Ohio (Harris Publishing Co., 1985) lists 112 industrial establishments in Geauga County. Only 20 of these establishments employ 100 or more people. Industry is diversified and includes publishing, sand and gravel quarrying, and manufacturing of machinery and rubber, plastic, machine, food, concrete, and maple products.

In 1980, 6.9 Mgal/d (million gallons per day) of water were used in Geauga County. Of this $6.9 \mathrm{Mgal} / \mathrm{d}, 5 \mathrm{Mgal} / \mathrm{d}$ was ground water. Fifty-nine percent of the ground-water use was for rural domestic and livestock supply, 39 percent was for public supply, and 2 percent was for manufacturing (Eberle and McClure, 1984). 
The study area is located in the Glaciated Allegheny Plateau section of the Appalachian Plateau physiographic province (Fenneman, 1938). Bedrock generally controls the topography within Geauga County. The bedrock surface, however, has been modified and covered by glacial deposits. The highest altitudes in the County are about $1,360 \mathrm{ft}$ (feet) above sea level, whereas the lowest altitudes are near $860 \mathrm{ft}$ above sea level.

Three river systems drain Geauga County. The Cuyahoga, Chagrin, and Grand Rivers all have their headwaters within the County (fig. 1). These three rivers, in part, follow buried glacial valleys because glaciers or their meltwaters did not completely fill the existing valleys. As a result, residual depressions remained to control the courses of postglacial streams (Baker, 1964). The City of Akron, in Summit County, located just southwest of Geauga County, owns and operates two water-supply reservoirs within Geauga County. These two reservoirs are East Branch (on the east branch of the Cuyahoga River) and LaDue (on Bridge Creek, which is a principal tributary to the Cuyahoga River). A gain/loss study conducted by U.S. Geological Survey personnel in October 1980 indicated that all river reaches within the County gain water from the local groundwater-flow system.

Geauga County has a humid temperate climate. Average annual precipitation from 1931 to 1980 ranged from $39 \mathrm{in./yr}$ (inches per year) to $43 \mathrm{in./yr}$ (fig. 2) (L.J. Harstine, Ohio Department of Natural Resources, written commun., 1988). The spatial variation of precipitation within the County is related to the County's location at the northern edge of the Appalachian Plateau near Lake Erie; moisture-laden winds are forced to rise over the Portage escarpment to reach the Geauga County uplands. As a result, rain and snow fall heavily on the northern rim of the Appalachian Plateau parallel to the Portage escarpment (Baker, 1964).

\section{Geologic Setting}

The consolidated rocks considered in this investigation are Paleozoic in age and range from Devonian to Pennsylvanian (table 1, pl. 1). The oldest bedrock units that crop out in Geauga County are the Chagrin Shale, the Cleveland Member of the Ohio Shale, and the Bedford Shale. These pre-Berea sedimentary rocks (pl. 1.) crop out along the sides and bottoms of buried glacial valleys, but have few surface exposures within the study area (Baker, 1964).

The Berea Sandstone, which overlies the Bedford Shale, is a shallow marine and deltaic quartz sandstone that is relatively well sorted and ranges in texture from coarse to fine. It is typically $60 \mathrm{ft}$ thick within Geauga County and underlies younger bedrock throughout most of the County (Baker, 1964). 


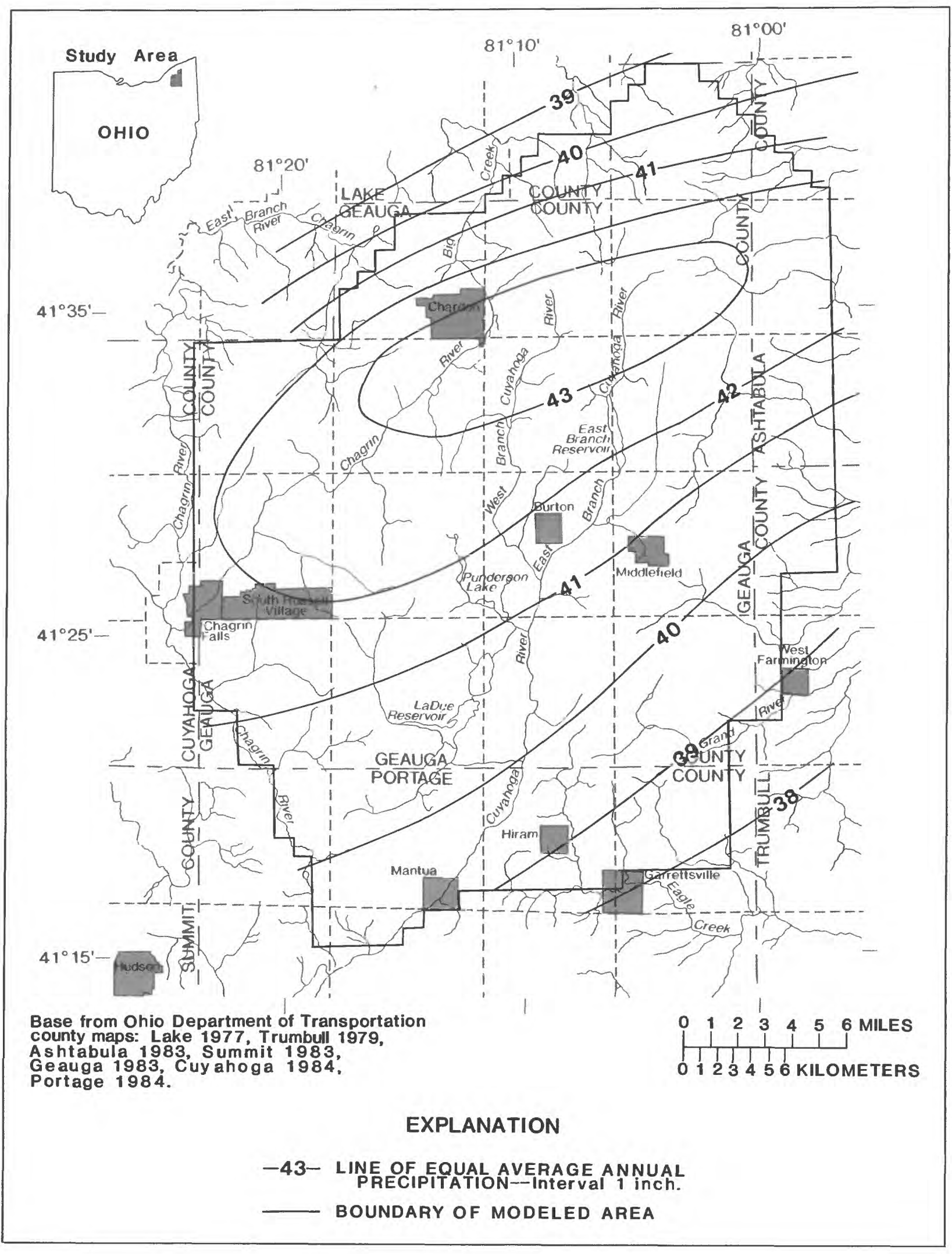

Figure 2.--Average annual precipitation, 1931-80 (from L. J. Harstine,

Ohio Department of Natural Resources, written commun., 1988). 


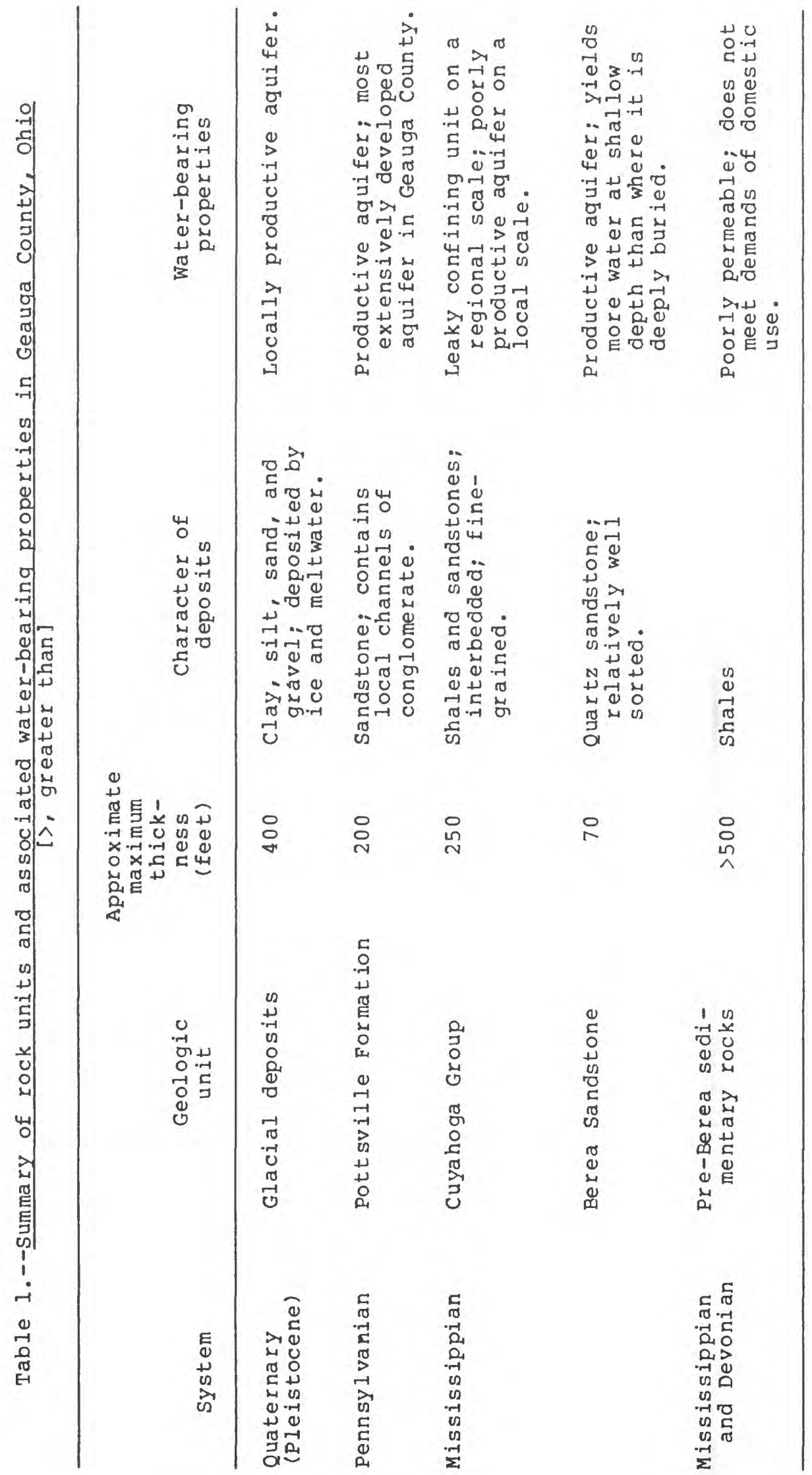


The Cuyahoga Group consists of the interbedded, shallow marine shales and sandstones above the Berea Sandstone and below the Pottsville Formation. The Cuyahoga Group is extensively exposed within Geauga County, particularly in the eastern part of the County. Because of erosion of its upper surface shortly after deposition (Baker, 1964), the Cuyahoga Group ranges from 60 to $250 \mathrm{ft}$ in thickness.

The Pottsville Formation is composed of terrestrial deposits and is the youngest of the consolidated rock units in Geauga County. Because of a gentle southward dip, the Pottsville Formation has a limited areal extent in the northern part of the County, where it commonly caps topographic high areas. In the southern part of the County its distribution is more extensive. The Pottsville Formation consists, in ascending order, of four members: the Sharon, the Connoquenessing Sandstone, the Mercer, and the Homewood Sandstone. The conglomerate unit of the Sharon Member is predominantly a sandstone that contains several long, thin north-south-trending conglomerate deposits. The overlying shale unit of the Sharon Member is thin or missing throughout most of Geauga County. The Connoquenessing Sandstone Member is the youngest member having a widespread distribution in Geauga County. In most areas, it is a quartz sandstone with a matrix of silt and clay. The Mercer Member occurs only in small scattered areas within the County and has been described as an interbedded sequence of shale, coal, underclay, limestone, and sandstone lenses in Portage County, south of Geauga County. The Homewood Sandstone Member, which overlies the Mercer Member, occurs only at the boundary between Troy and Parkman Townships where it is a coarse, friable, quartzose sandstone. The Pottsville Formation in Geauga County was mapped by Baker (1964) as one geologic unit (pl. 1).

During the Pleistocene Epoch or Ice Age, continental ice sheets covered much of North America during four separate ice advances. As a result, most of the bedrock in Geauga County is covered with glacial deposits. Sediment deposited directly by the ice is called till and commonly consists of poorly sorted, angular pebbles and boulders in a silty clay or clay matrix. Sediment deposited by glacial meltwater consists chiefly of sand and gravel having some degree of bedding, sorting, and rounding. The thickest sand and gravel deposits in Geauga County are in Auburn and Newbury Townships (fig. 1). Baker (1964) and Totten (1988) provide extensive descriptions of the glacial deposits within the County.

Because of the many glacial advances and retreats, Geauga County contains a network of intersecting buried valleys. The glacial deposits that fill these valleys were deposited by ice and meltwater and consist of a mixture of clay, silt, sand, and gravel. The Ohio Drilling Company (1971) evaluated several buried valleys in northeastern Ohio for potential ground-water supplies. Their testing consisted of seismic profiling to establish the location of the buried valleys and test drilling to determine the types and thicknesses of the glacial deposits present in the buried valleys. Aquifer tests were performed in selected locations. Figure 3 illustrates the locations of the buried valleys as determined by the Ohio Drilling Company (1971). 


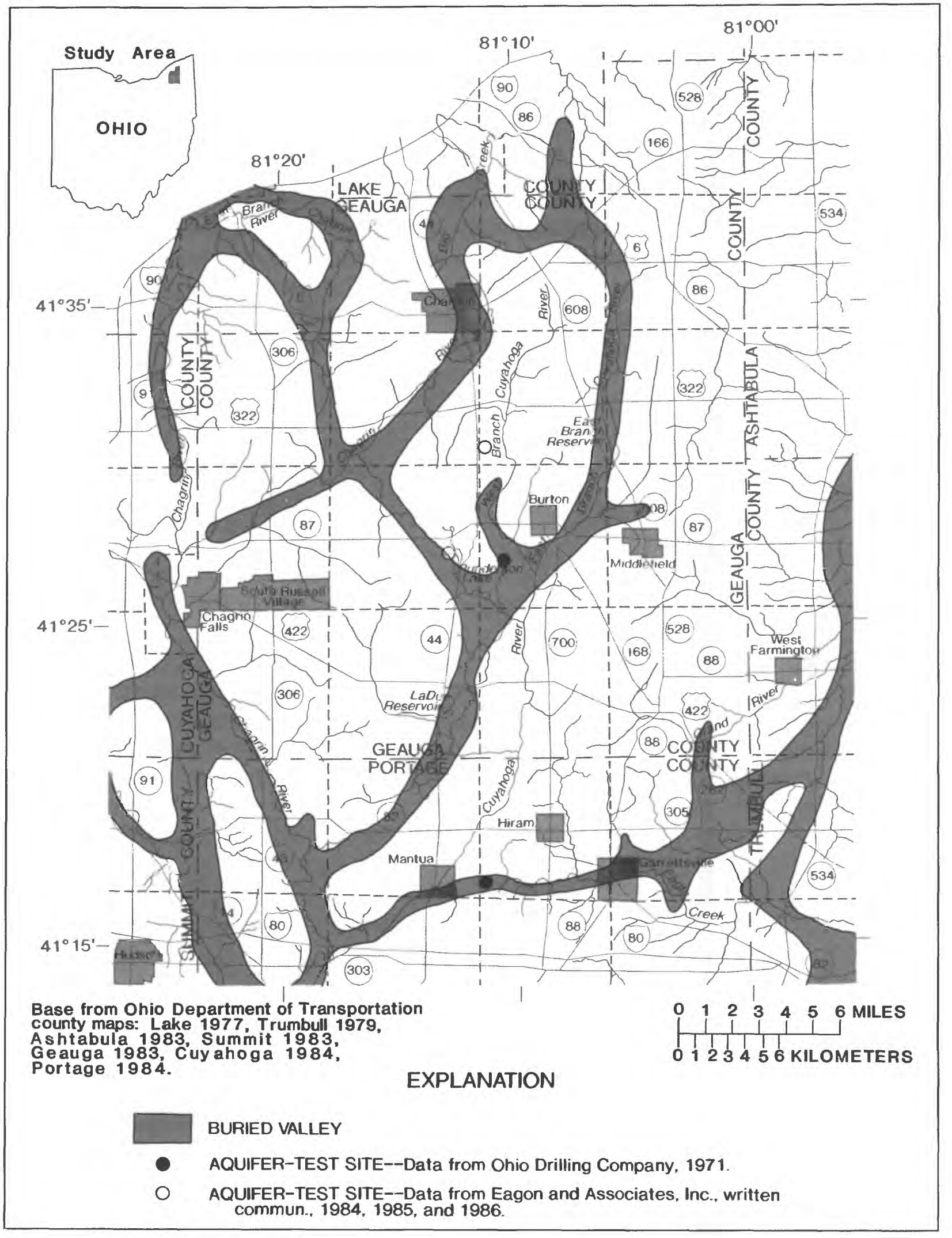

Figure 3.--Location of buried valleys (Ohio Drilling Company, 1971) and aquifer-test sites. 
Five geologic sections were constructed as part of the current investigation to illustrate the relation between the bedrock units and the overlying glacial deposits (pl. 2). Variations in the composition of the glacial deposits are not distinguished on these sections. The geologic sections were compiled from numerous maps. These maps include 7-1/2 minute U.S. Geological Survey topographic quadrangle maps; bedrock geologic maps (Baker, 1964; Winslow and White, 1966); top-of-bedrock maps (Vormelker, 1981; Risser, 1983; Risser in White and Totten, 1979; Vormelker in White, 1971; and Vormelker in White, 1980); and maps of the structure on the Berea Sandstone (Gray, 1981a and 1981b). The generalized top-of-bedrock contours for Geauga and Portage Counties presented on the bedrock geologic map compiled from Baker (1964) and Winslow and White (1966) in plate 1 were not used in construction of these geologic sections. Instead, more recent and detailed top-of-bedrock maps for Geauga and Portage Counties by Vormelker (1981) and Risser (1983) were used. As a result, bedrock contacts in the geologic sections differ slightly from those shown in plate 1.

\section{METHODS OF STUDY}

\section{Water-Level Measurement}

Water levels were measured as many as five times from August 1985 through November 1986 in 104 wells located within Geauga County (fig. 4, table 2, at back of report). Water levels also were measured once in a network of 21 wells in adjacent parts of Lake, Ashtabula, Trumbull, and Portage Counties (fig. 4, table 2) in late January and early February 1986. Water levels in the wells outside Geauga County were used to extend the water-level maps to the natural boundaries of the ground-water-flow system. Each well selected for use in this study produces water from a single aquifer or from the regional confining unit. Well logs, availability of geologic information, and well accessibility were considered during well selection. Water levels were measured in 48 of these wells during one or both of the previous studies.

\section{Water-Quality Sampling}

A reconnaissance survey was made of 98 well waters to determine the range of specific conductance in the ground-water-flow system in Geauga County. These data then were used as one of the criteria to select wells to be sampled for detailed analysis of ground-water chemistry. The final set of wells selected for sampling included waters with a range of specific conductance from relatively low to relatively high values, as determined from the reconnaissance survey. Such an approach helped ensure that the entire range of water types present in the County was included in the set of wells sampled for detailed chemical analysis. The final set of wells also included several waters from each of the aquifers and the regional confining unit, as well as waters from recharge, 


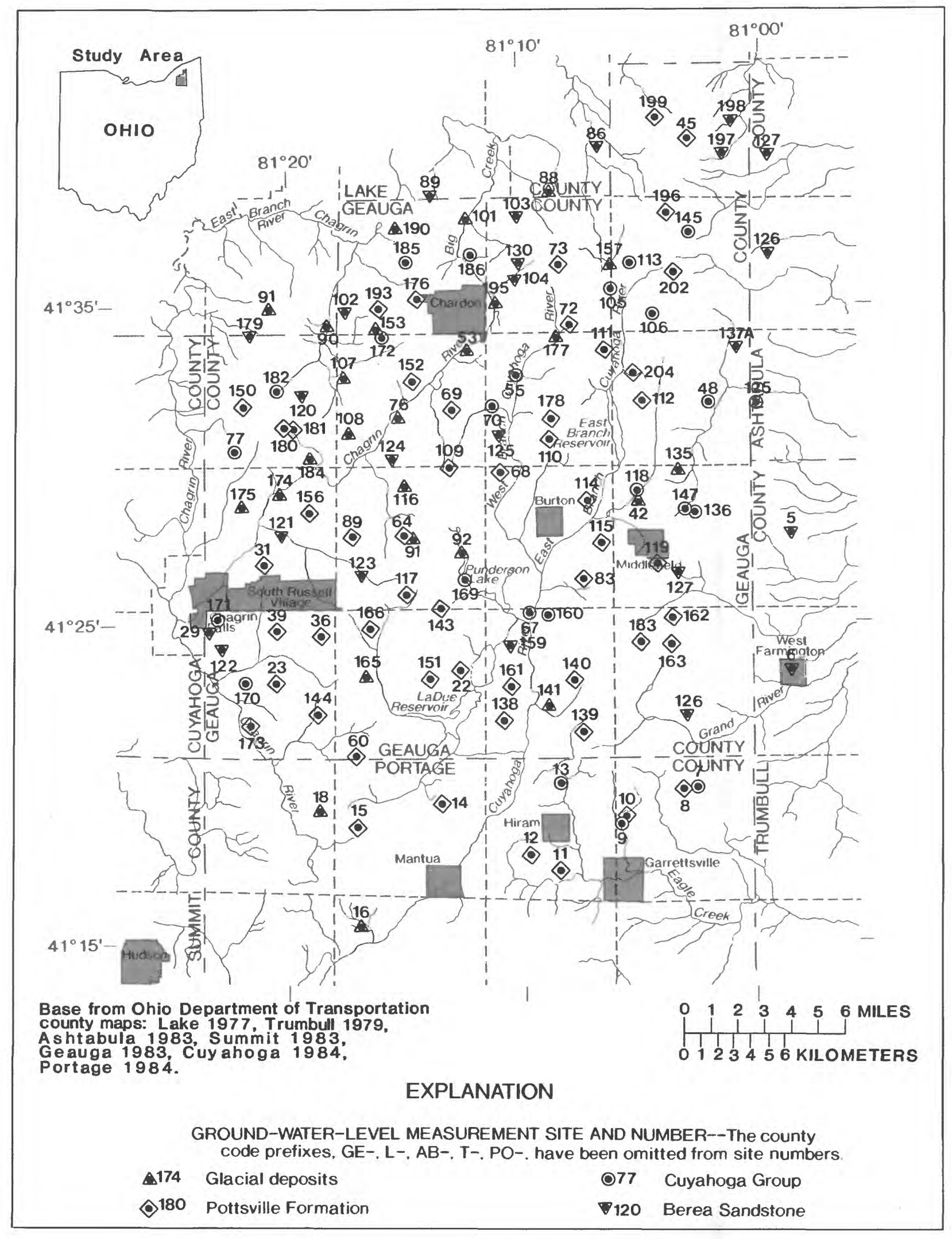

Figure 4.--Sites at which ground-water levels were measured 
discharge, and intermediate positions within the flow system. The specific-conductance measurements were made using a four-parameter probe (specific conductance, $\mathrm{pH}$, dissolved oxygen concentration, and temperature) in a flow chamber and were taken while the well was discharging and after measurements of these properties had stabilized.

Thirty-eight domestic or commercial wells within Geauga County were sampled to determine background water quality (fig. 5). Each well sampled produces water from a single aquifer or the regional confining unit. In addition, four domestic well waters were collected for determination of oilfield or gasfield brine contamination. Three of these wells (GE-205, GE-206, and GE-207) were sampled because of homeowner complaints registered with the Division of Oil and Gas of the Ohio Department of Natural Resources (fig. 5). The other well (GE-113) was sampled for control (fig. 5). Final well selection was based upon an inspection of the plumbing system at each well to insure that a representative sample of the ground water in the production zone could be obtained. Wells that were affected by a water-treatment system or subject to surface leakage were not sampled. Twelve of the 42 wells sampled during the current study were sampled during one or both of the previous studies. In addition, brine was collected from three oil- or gas-production wells in Geauga or Summit County (fig. 5).

The volume of water in the well bore of each well was calculated before collecting water samples. If a pressure tank was present and could not be bypassed, this volume also was calculated and added to the well-bore volume. An amount of water equal to three times the volume in the system was purged before the sample was taken. Well discharge was volumetrically estimated, and a fully enclosed flow chamber was used to monitor readings of $\mathrm{pH}$, dissolved oxygen concentration, specific conductance, and temperature. Samples were taken when purging was complete or when successive readings of field parameters stabilized. An inline filter equipped with a $0.45 \mu \mathrm{m}$ (micrometer) membrane was used to collect samples for dissolved metals. Sampling lines and the flow chamber were cleaned between sites with a detergent solution followed by a tap-water rinse.

Samples were preserved as required according to procedures in Greeson and others (1977), Brown and others (1970), and U.S. Geological Survey, Office of Water Data Coordination (1977), and analyzed by the U.S. Geological Survey Central Laboratory in Denver, Colorado. Specific conductance, $\mathrm{pH}$, dissolved oxygen concentration, temperature, fecal coliform, fecal streptococci, and alkalinity were determined in the field. Hydrogen sulfide concentration was determined in the district lab in Columbus, Ohio.

Samples of oilfield or gasfield brine were collected directly from the wellhead in acidwashed $\left(\mathrm{HNO}_{3}\right)$ 1-gallon glass containers. If oil was present in the sample, the two liquid phases (oil and water) were allowed to separate, the oil was decanted, and the brine was promptly filtered. Brines were pressure filtered, using argon gas and a $0.1-\mu \mathrm{m}$ filter. 


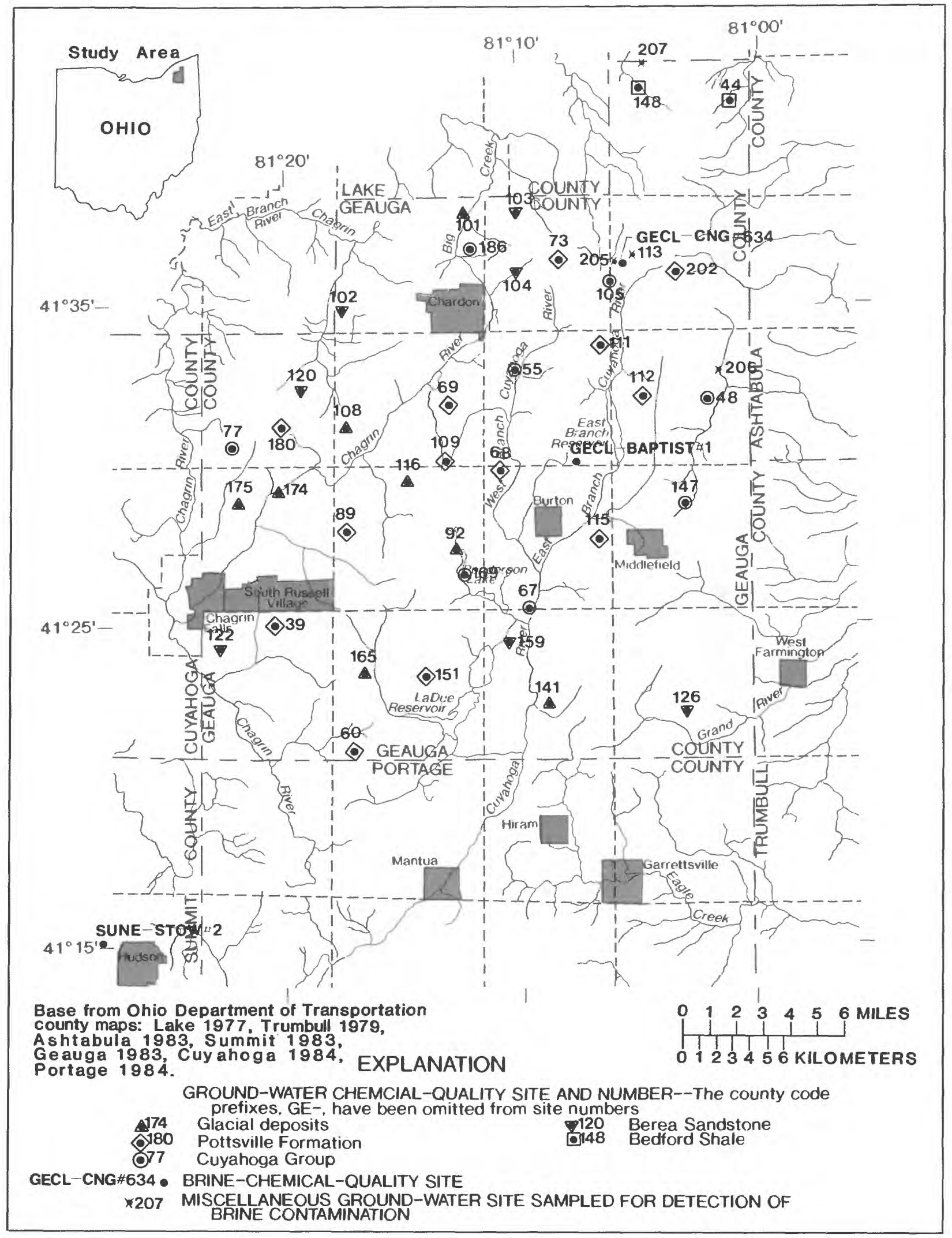

Figure 5.--Wells sampled for water-quality analysis 
Brine samples were preserved as required (Fishman and Friedman, 1989) and custom analyzed by the U.S. Geological Survey Central Laboratory in Denver, Colo. Specific conductance was determined using a Wheatstone Bridge in the district lab in Columbus, Ohio. Specific gravity also was determined in the district lab, whereas $\mathrm{pH}$ and alkalinity concentrations were determined in the field.

\section{Determination of Ground-Water Flow Paths, Residence Times, and Water-Level Changes}

The McDonald and Harbaugh (1984) finite-difference program was used to construct a steady-state, three-dimensional model of the ground-water-flow system in Geauga County and vicinity. Results of the calibrated model were used to compute a threedimensional velocity field for each aquifer and confining layer in the model. These velocity data were used in the particle-tracking program MODPATH (Pollock, 1989) to determine advective ground-water flow paths and residence times for various particles of ground water within the flow system. The calibrated model also was used to predict the steady-state response of the flow system to increased ground-water development.

\section{GEOHYDROLOGY}

\section{Aquifers and Confining Units}

\section{Glacial Deposits}

Glacial deposits are most commonly used as a source of water in the lowlands within Geauga County where the deposits are the thickest. Most domestic wells that produce water from the glacial deposits within the County are completed without a well screen. The wells are cased to the bottom of the drilled hole, thus allowing water to enter the well only from the open bottom of the casing. This type of well construction allows less water to enter the well than if the well were screened in the entire saturated thickness of the aquifer. The reported yields of domestic wells included in this study that produce from glacial deposits range from 7 to $34 \mathrm{gal} / \mathrm{min}$ (gallons per minute). One well screened in the glacial deposits in the Middlefield well field (GE-42, fig. 4) produces approximately $410 \mathrm{gal} / \mathrm{min}$.

The Ohio Drilling Company (1971) has conducted test drilling within Geauga County to determine the type of glacial deposits in the buried valleys. The buried valley extending from Chardon to Chagrin Falls (fig. 3 ) is filled primarily with silt and clay with intermittent thin layers of sand and gravel. The Ohio Drilling Company (1971) noted that the sand and gravel layers within this valley are potentially useful for development of domestic and small commercial wells. The Village of Chardon has developed a well field in an anomalously thick sand and gravel layer within the buried valley. 
Drillers' logs from domestic wells indicate the presence of sand and gravel in the buried valley that trends southwestward from Burton (fig. 3) to northern Portage County. The Ohio Drilling Company (1971) performed an aquifer test near Burton where more than $100 \mathrm{ft}$ of sand and gravel fill the valley. The following table summarizes the aquifer-test data available for the glacial deposits within the study area.

\begin{tabular}{|c|c|c|c|}
\hline $\begin{array}{l}\text { Location of } \\
\text { measurement } \\
\text { (fig. 3) }\end{array}$ & $\begin{array}{l}\text { Transmis- } \\
\text { sivity } \\
\text { (square } \\
\text { feet per } \\
\text { day) }\end{array}$ & $\begin{array}{l}\text { Horizontal } \\
\text { hydraulic } \\
\text { conductivity } \\
\text { (feet per } \\
\text { day) }\end{array}$ & Source of data \\
\hline Near Burton & 10,000 & 106 & $\begin{array}{l}\text { The Ohio Drilling } \\
\text { Company (1971) }\end{array}$ \\
\hline East of Mantua & 7,400 & 150 & $\begin{array}{l}\text { The Ohio Drilling } \\
\text { Company (1971) }\end{array}$ \\
\hline $\begin{array}{l}\text { Punderson } \\
\text { State Park }\end{array}$ & 2,100 & -1 & $\begin{array}{l}\text { Eagon and Associ- } \\
\text { ates (written } \\
\text { commun., 1986) }\end{array}$ \\
\hline
\end{tabular}

${ }^{1}$ Dash indicates no data available.

The buried valley that extends westward from Garrettsville and underlies Mantua (fig. 3) locally contains thick deposits of sand and gravel. The Ohio Drilling Company (1971) noted that high-capacity wells could be developed in this valley. They performed an aquifer test just east of Mantua. Although glacial deposits are $150 \mathrm{ft}$ thick in the valley at the site of the aquifer test, it was thought that the thickest part of the aquifer consists of sand and gravel averaging only $50 \mathrm{ft}$ thick.

In addition to hydraulic characteristics reported by the Ohio Drilling Company (1971), Eagon and Associates (written commun., 1986) report a transmissivity value for a well completed in the glacial deposits in Punderson State Park, Newbury Township (fig. 3).

\section{Pottsville Formation}

At the regional scale, the interbedded sandstones of the Pottsville Formation may be considered to compose a single hydrostratigraphic unit. This bedrock aquifer is the most extensively used aquifer within the County. A typical well that produces from bedrock within the County is cased into the top of the bedrock unit from which it produces; the remainder of the well is completed as an open hole. Such wells rarely penetrate the entire thickness of the producing aquifer. Reported yields of domestic wells included in this study that produce from the Pottsville Formation range from 5 to $35 \mathrm{gal} / \mathrm{min}$. 
Few aquifer tests have been conducted in the Pottsville Formation within the study area. The various consultants who have conducted the tests have noted that most of the wells in which aquifer tests have been conducted are completed in a fracture within the aquifer. Much of the water in the Pottsville Formation moves along such fractures and joints in the rock; many of these fracture openings have been enlarged by weathering or solution where the aquifer is at or near land surface (Baker, 1964).

Winslow and White (1966) report a transmissivity for the Sharon Member and the Connoquenessing Sandstone Member calculated from an aquifer test conducted in Portage County. In addition, Eagon and Associates, Inc. (written commun., 1984 and 1985) report transmissivities for the Pottsville Formation on the basis of three aquifer tests performed at the County facilities on State Route 44 north of State Route 87 in Geauga County (fig. 3). Sedam (1973) determined transmissivity and horizontal hydraulic conductivity for the Sharon and Connoquenessing Members of the Pottsville Formation on the basis of specific-capacity data derived from drillers' logs. Because the Mercer and Homewood Sandstone Members have a very limited distribution within Geauga County, they are not hydrologically important within the County (Baker, 1964). The above data are summarized in the following table.

\begin{tabular}{|c|c|c|c|}
\hline $\begin{array}{l}\text { Location of } \\
\text { measurement(s) } \\
\text { (fig. 3) }\end{array}$ & $\begin{array}{l}\text { Transmis- } \\
\text { sivity } \\
\text { (square } \\
\text { feet per } \\
\text { day) }\end{array}$ & $\begin{array}{l}\text { Horizontal } \\
\text { hydraulic } \\
\text { conductivity } \\
\text { (feet per } \\
\text { day) }\end{array}$ & Source of data \\
\hline $\begin{array}{l}\text { Ravenna Arsenal, } \\
\text { Portage County }\end{array}$ & 1,700 & $-^{1}$ & $\begin{array}{l}\text { Winslow and White } \\
\text { (1966) }\end{array}$ \\
\hline $\begin{array}{l}\text { County facilities, } \\
\text { Geauga County }\end{array}$ & $100-1,800$ & - & $\begin{array}{l}\text { Eagon and Associates, } \\
\text { Inc. (written commun. } \\
1984 \text { and 1985) }\end{array}$ \\
\hline $\begin{array}{l}\text { Average or range } \\
\text { for Connoque- } \\
\text { nessing Member } \\
\text { of the Pottsville } \\
\text { Formation, } \\
\text { Geauga County }\end{array}$ & 290 & $<1-40$ & Sedam (1973) \\
\hline $\begin{array}{l}\text { Average or range } \\
\text { for Sharon Member } \\
\text { of the Pottsville } \\
\text { Formation, Geauga } \\
\text { County }\end{array}$ & 450 & $5-65$ & Sedam (1973) \\
\hline
\end{tabular}




\section{Cuyahoga Group}

None of the shale or sandstone formations within the Cuyahoga Group is highly permeable. Because intergranular openings within bedrock of the Cuyahoga Group are extremely small, most ground water moves along joints, bedding planes, and fractures. As a result, these formations have been treated as a single hydrostratigraphic unit (Baker, 1964). The importance of the Cuyahoga Group to the regional ground-water-flow system is that these formations impede vertical ground-water movement and are considered to represent a leaky confining unit on a regional scale. On a local scale, however, the Cuyahoga Group can supply sufficient quantities of water for domestic use.

On the basis of drillers' logs, the domestic wells included in this study that produce from the Cuyahoga Group have yields ranging from 5 to $30 \mathrm{gal} / \mathrm{min}$. However, unlike wells completed in the other aquifers, most wells completed in the Cuyahoga Group display significant drawdown during their well-acceptance test. In fact, drawdown was complete in one-fourth of the wells included in this study. Because recovery of wells completed in the Cuyahoga Group is slow, most homeowners rely on large storage tanks and storage of water within the well bore for their water supply.

No aquifer-test data are available for the Cuyahoga Group within Geauga County. However, Jenkins (1987) estimated the average horizontal hydraulic conductivity to be $0.7 \mathrm{ft} / \mathrm{d}$ (feet per day) for the Cuyahoga Group in Munson Township (fig. 1) from specific-capacity data derived from drillers' logs. Horizontal hydraulic conductivity was estimated to be around $0.4 \mathrm{ft} / \mathrm{d}$ by use of specific-capacity data obtained from logs of wells included in this study and techniques developed by Theis and others (1963). Data from wells that penetrate only a small portion of the Cuyahoga Group or that experience significant drawdown were not used for calculation of horizontal hydraulic conductivity. No data are available to estimate the vertical hydraulic conductivity of the Cuyahoga Group.

\section{Berea Sandstone}

The Berea Sandstone has not been developed extensively as an aquifer throughout most of Geauga County because it is too deeply buried to be developed economically as a domestic water-supply source (pl. 2). In addition, the sandstone yields more water at shallow depth than where it is deeply buried because weathering has enlarged joints within the formation near the land surface (Baker, 1964).

The reported yields of domestic wells included in this study that produce from the Berea Sandstone range from 4 to $25 \mathrm{gal} / \mathrm{min}$. Rau (1969) reports an average yield of 18 $\mathrm{gal} / \mathrm{min}$ for 655 wells completed in the Berea Sandstone in northeastern Ohio. He notes that this value does not necessarily represent maximum yield available because most 
domestic wells do not fully penetrate the aquifer and because yield often is limited by the capacity of the pump installed in the well. Rau (1969) also reports an average transmissivity of approximately $500 \mathrm{ft}^{2} / \mathrm{d}$ and a corresponding average horizontal hydraulic conductivity of $8 \mathrm{ft} / \mathrm{d}$. He notes that transmissivities are lower near the outcrop area where the sandstone is relatively thin. In addition, Rau (1969) states that one of the most important factors to be considered in locating sites for ground-water development from the Berea Sandstone is the availability of recharge to the aquifer.

\section{$\underline{\text { Recharge }}$}

Precipitation is the source of virtually all ground water within the study area. However, as little as 10 percent of the 39 to 43 in./yr of precipitation actually recharges the aquifers in some parts of Geauga County. Much of the precipitation runs off directly into streams as a result of land-surface relief and the relatively impermeable glacial till that covers most of the area. In addition, some water is returned to the atmosphere through evaporation and transpiration by plants.

Recharge rates were estimated for northeastern Ohio by Pettyjohn and Henning (1979) by use of base-flow separation of stream hydrographs. Their recharge estimates range from 2 to $8 \mathrm{in}$./yr of recharge for Geauga County. Most of the County is thought to receive only 2 to 4 in./yr of recharge. However, estimates for deposits along the Cuyahoga River were between 4 and $6 \mathrm{in}$./yr of recharge and were between 6 and $8 \mathrm{in} . / \mathrm{yr}$ for some deposits along the Chagrin River (Pettyjohn and Henning, 1979).

Surficial aquifers such as the glacial deposits and the Pottsville Formation are recharged by precipitation infiltrating directly into the deposits. Recharge from precipitation to the underlying Cuyahoga Group and Berea Sandstone occurs indirectly as vertical leakage of ground water from overlying aquifers, except along their outcrop belts. Conditions for recharge to the Cuyahoga Group and Berea Sandstone are favorable where their outcrop areas are relatively wide, the bedrock is fractured and jointed near the surface, and where there is low topographic relief. If the outcrops are directly overlain by permeable glacial deposits that facilitate infiltration of water at high rates during precipitation, recharge conditions are even more favorable. Rau (1969) notes that where the Berea Sandstone is overlain by a thick section of the Cuyahoga Group, local wells may not receive sufficient recharge to sustain even moderate production rates.

\section{Ground-Water Levels and Flow Directions}

Ground-water-level maps (figs. 6-8) were constructed from measurements made during January and February 1986. Figure 6 depicts water levels in the Pottsville Formation and the glacial deposits. The Pottsville Formation is the surficial aquifer where 


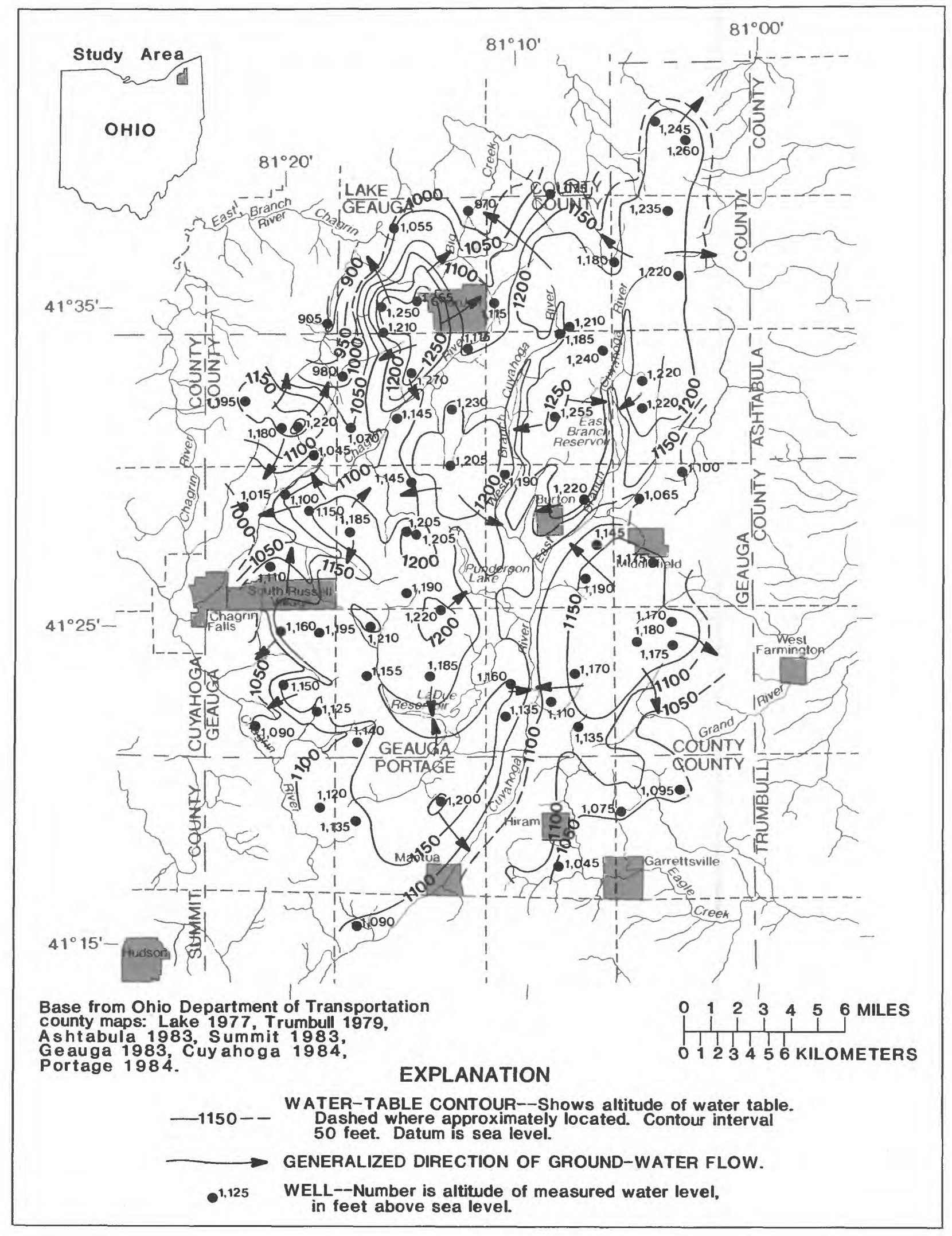

Figure 6.--Ground-water levels in the Pottsville Formation and the glacial deposits, January-February 1986. 


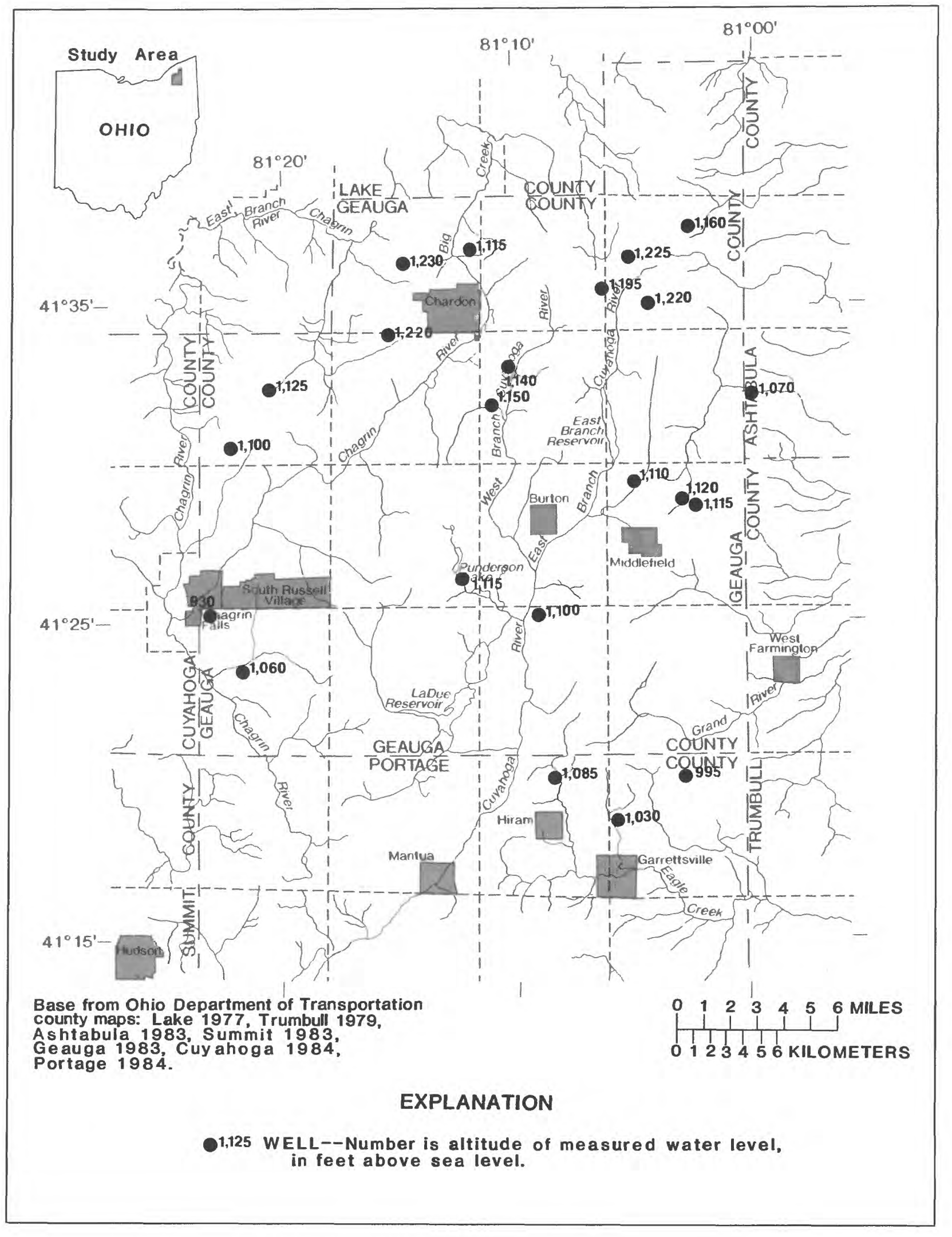

Figure 7.--Ground-water levels in the Cuyahoga Group, January-February 1986. 


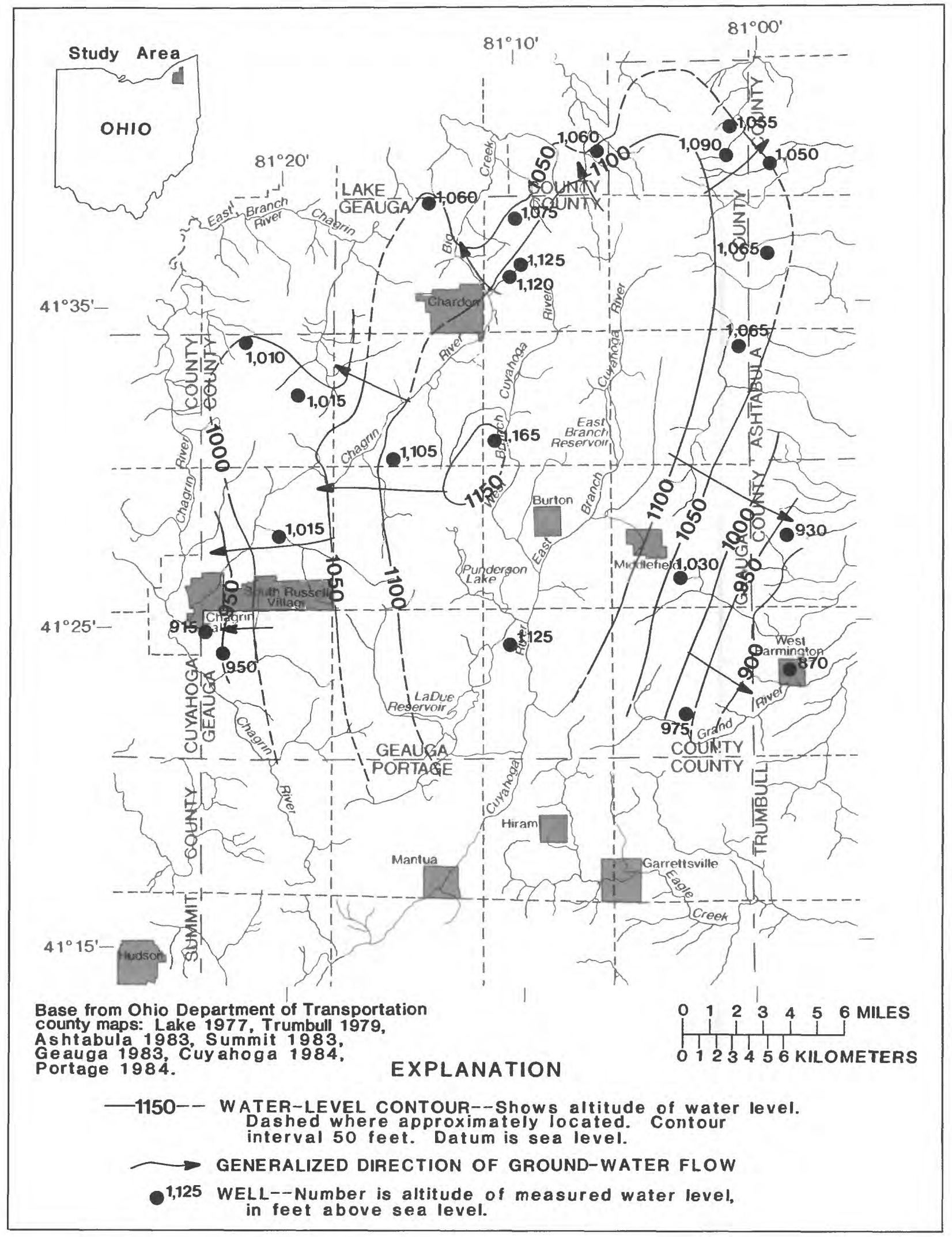

Figure 8.--Ground-water levels in the Berea Sandstone, January-February 1986. 
glacial deposits are unsaturated on the highlands. The glacial deposits compose the surficial aquifer in the lowlands where the Pottsville Formation is discontinuous. In general, the water table follows the configuration of the land surface in Geauga County.

Ground water moves from recharge areas to discharge areas generally perpendicular to lines of equal altitude of the water surface (fig. 6). Water in the surficial aquifers within the study area locally discharges into the Cuyahoga River, Chagrin River, Grand River, or their tributary streams as illustrated in figure 6. Where bedrock knobs are present, such as in Chester and Chardon Townships, flow in the surficial aquifers is radial.

January-February 1986 ground-water levels in the Cuyahoga Group are depicted in figure 7. The predominant direction of ground-water flow in the Cuyahoga Group is vertically downward from the Pottsville Formation toward the Berea Sandstone. This is because the hydraulic head in the Pottsville Formation generally is greater than that in the Cuyahoga Group and in the underlying Berea Sandstone. The ground-water levels in figure 7 were not contoured because of the large vertical-flow component in the Cuyahoga Group. Some ground water, however, does discharge as seeps along outcrops where the Cuyahoga Group is exposed at land surface. These outcrops are principally along the northern, eastern, and western boundaries of the study area.

A potentiometric surface map was constructed for the Berea Sandstone aquifer from 22 water-level measurements made during January and February 1986 (fig. 8). The distribution of water-level data is concentrated near the outcrops of the Berea Sandstone where the aquifer is most developed. Few wells were available for measurement where the aquifer is deeply buried. Although the Berea Sandstone dips to the south at 10 to 20 $\mathrm{ft}$ per mile, the available water-level data indicate that flow within the aquifer is not simply in the down-dip direction. Rather, ground-water flow within the Berea Sandstone appears to be toward the outcrops of the formation along the northern, eastern, and western boundaries of the study area.

\section{Long-Term Water-Level Changes}

Water levels were measured in 125 wells during the current study. Water levels also were measured in 48 of these wells during the data-collection phase of the two previous U.S. Geological Survey studies conducted in cooperation with the Geauga County Sanitary Engineering Department (Nichols, 1980). The water-level data from these 48 wells were used to assess long-term water-level changes in the aquifers within Geauga County.

Water levels in nine of the wells included in the current study that produce from glacial deposits were measured during the 1978 and (or) 1980 studies. Data for seven of these wells were plotted to illustrate long-term water-level changes in the glacial deposits (fig. 9). Water levels from the additional two wells were not plotted because 


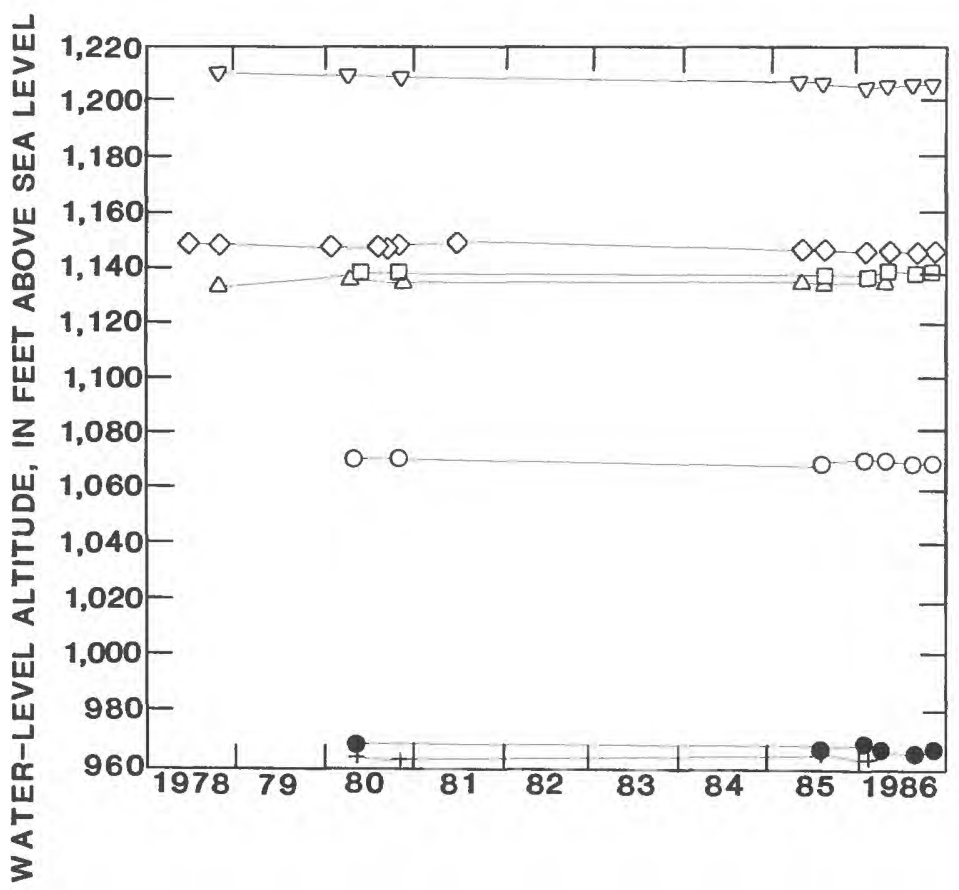

\section{EXPLANATION}

$\triangle$ GE-92

$\nabla$ GE-91

口 GE-116

$\diamond$ GE-76

- GE-101

- GE-108

+ GE-107

Figure 9.--Long-term ground-water-level changes in the glacial deposits (lines connecting the symbols are for visual reference only and do not imply continuity of data).

岁

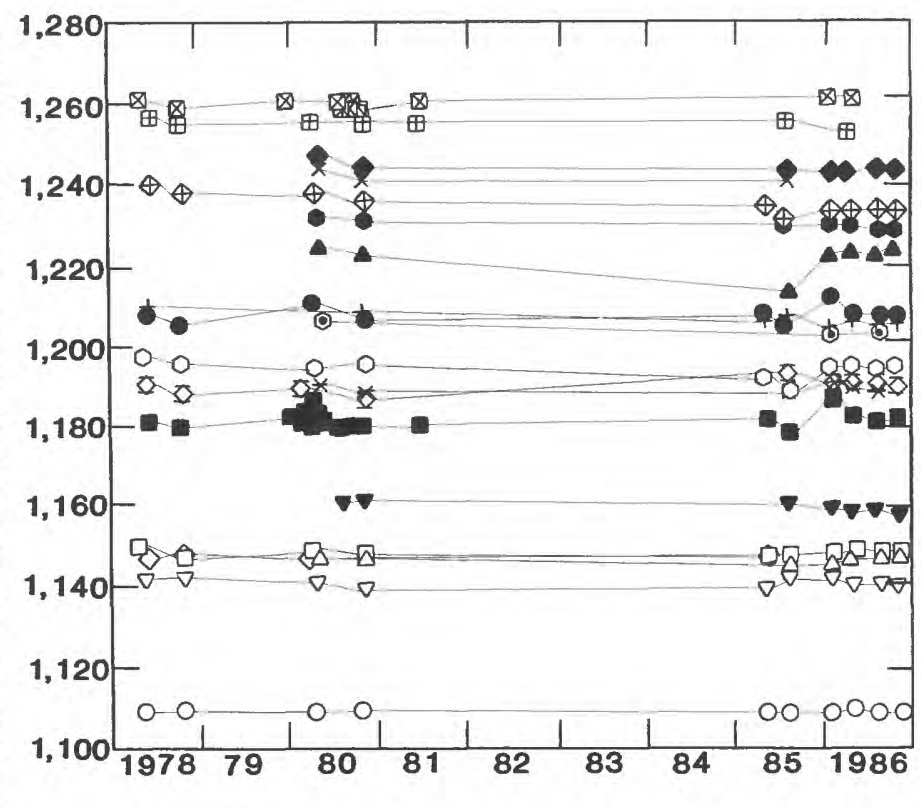

EXPLANATION

$\triangle$ GE-115 $\bullet$ GE-72

$\nabla$ GE-60 $\rightarrow$ GE-73

$\square$ GE-23 $\otimes$ GE-45

$\diamond$ GE-22 * GE-117

O GE-36 GE-83

० GE-31 $~$ GE-119

$+\mathrm{GE}-64 \backsim \mathrm{GE}-114$

- GE-68 ๑ GE-109

$\oplus$ GE-69 $\times$ GE-110

- GE-112 $\bullet$ GE-111

Figure 10.--Long-term ground-water-level-changes in the Pottsville Formation (lines connecting the symbols are for visual reference only and do not imply continuity of data). 
the data are not representative of natural water-level fluctuations in the glacial aquifer. One of these wells is an observation well in the Chardon well field, and the other is an observation well in the Middlefield well field. Much of the water-level data for these two wells was collected when the nearby production wells were operating. Available data indicate that no long-term water-level changes have occurred in the seven wells included in figure 9 since 1978 or 1980.

Water levels were measured in 20 of the wells completed in the Pottsville Formation during the current study and the 1978 and (or) 1980 studies. The available long-term water-level data for the Pottsville Formation indicate that less than $5 \mathrm{ft}$ of water-level fluctuation has occurred since 1978 or 1980 in 15 of these wells (fig. 10). Water levels in four of the five additional wells (GE-36, 68, 72, and 114) fluctuated more than $5 \mathrm{ft}$ since 1978 or 1980 . However, no long-term rises or declines are apparent from these data. Figure 10 illustrates a small but steady decline in water levels in well GE-69 (fig. 4). The overall decline in this well is only $8 \mathrm{ft}$, which is less than seasonal fluctuations detected in most of the other Pottsville Formation wells that were measured. However, well GE-69 does not appear to be sensitive to seasonal changes in recharge; less than $1 \mathrm{ft}$ of water-level change was measured in this well during the 1986 calendar year.

Water-level data from the 1978 and (or) 1980 studies and the current study are available for seven wells completed in the Cuyahoga Group. Long-term water-level fluctuations were greater in a few of the wells completed in the Cuyahoga Group (fig. 11) than in those completed in the glacial deposits or the Pottsville Formation, although no longterm rises or declines were observed. Water levels fluctuated most in well GE-105 (fig. 4). This may be due, in part, to the slow recovery of the well after pumping; the driller noted on the well log that complete drawdown occurred in the well during the acceptance test. During the period of study, water levels in well GE-48 (fig. 4) fluctuated only $1 \mathrm{ft}$.

Water-level data from 1978 and (or) 1980 and 1985 to 1986 are available for twelve wells completed in the Berea Sandstone and are plotted in figure 12. Although water levels fluctuated up to $27 \mathrm{ft}$ in some wells, none of the wells exhibited a notable longterm increase or decrease

\section{GROUND-WATER QUALITY}

\section{Reconnaissance Specific-Conductance Survey}

Specific conductance, a measure of the ability of water to conduct an electrical current, is related to the amount, type, and charge of dissolved ions present in a water sample. Thus, specific conductance, which is measured easily in the field, can be used to approximate the total dissolved solids (TDS) concentration of a water sample because 
$\triangle$ GE-67

$\nabla$ GE-118

ㅁ GE-77

$\checkmark$ GE-48

- GE-105

- GE-113

+ GE-106

Figure 11.--Long-term ground-water-level changes in the Cuy ahoga Group (lines connecting the symbols are for visual reference only and do not imply continuity of data).

岁

1,170

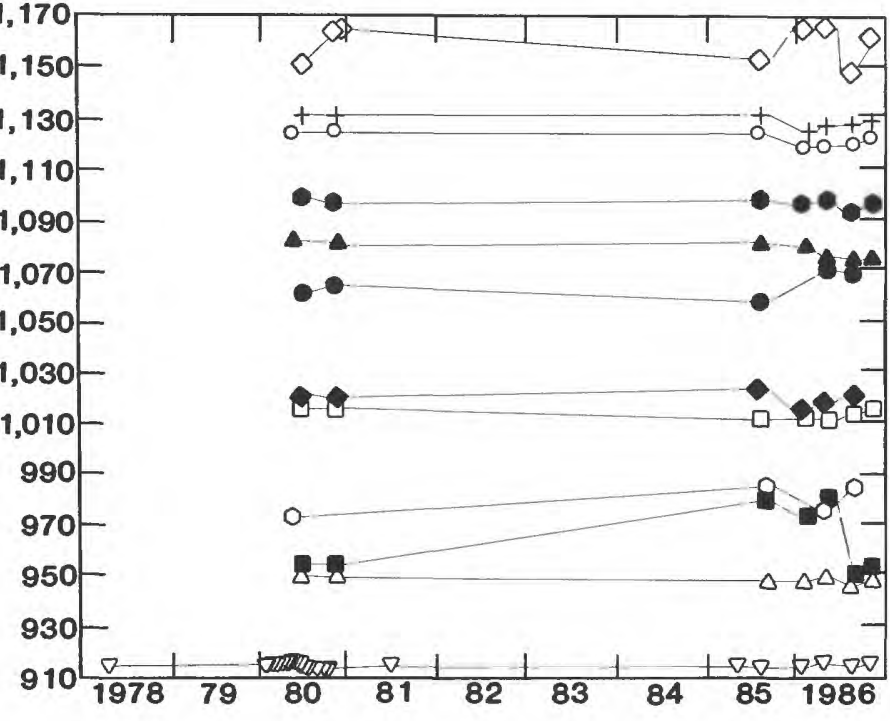

EXPLANATION

$\triangle$ GE-122

$\nabla$ GE-29

․ GE-121

$\diamond$ GE-125

- GE-102

O GE-104

+ GE-130

- GE-103

- GE-120

- GE-124

- GE-123

- GE-126

Figure 12.--Long-term ground-water-level changes in the Berea Sandstone (lines connecting the symbols are for visual reference oniy and do not imply continuity of data). 
specific conductance generally increases as TDS increases (Hem, 1985). Potable ground waters have specific conductances ranging from 30 to $2,000 \mathrm{uS} / \mathrm{cm}$ (microsiemens per centimeter at 25 degrees Celsius), whereas rainwater has specific conductances ranging from 5 to $30 \mathrm{uS} / \mathrm{cm}$, and sea water has a specific conductance of about $50,000 \mu \mathrm{S} / \mathrm{cm}$ (Davis and DeWeist, 1966).

Figure 13 shows the relation between specific conductance and TDS from 29 wells that were sampled in 1986 for detailed chemical analysis. This figure includes water samples from each of the aquifers and the regional confining unit, and includes waters from different relative positions within the flow system. It does not include data from well waters that were determined to be affected by road salt and (or) oilfield and gasfield brine during the course of the project. The equation describing the best-fit linear regression line (fig. 13), which has a correlation coefficient of 0.980 , is

$$
\mathrm{TDS}=0.670(\mathrm{SpC})-24.8,
$$

where

TDS is total dissolved solids concentration ( $\mathrm{mg} / \mathrm{L}$ ), and $\mathrm{SpC}$ is specific conductance (uS/cm).

Either this equation or the line on figure 13 can be used to estimate the TDS concentration of a ground-water sample from Geauga County if the specific conductance for the sample has been measured. Caution should be exercised when using this equation to estimate TDS, particularly when using specific-conductance values greater than $1,000 \mu \mathrm{S} /$ $\mathrm{cm}$ or less than $150 \mu \mathrm{\mu S} / \mathrm{cm}$.

\section{Spatial Variations in Quality}

The chemical character of a water indicates the relative abundance of the major anions (bicarbonate, sulfate, and chloride) and cations (calcium, magnesium, and sodium), and provides a means of classifying and distinguishing waters by type and origin. It is determined by converting milligrams per liter $(\mathrm{mg} / \mathrm{L}$ ) or parts per million (ppm) concentrations of the major ionic species into milliequivalents per liter (meq/L) or equivalents per million (epm) concentrations. This is accomplished by dividing the $\mathrm{mg} / \mathrm{L}$ or ppm concentration by the gram equivalent weight (the gram formula weight divided by the valence of the ionic specie). The chemical character of the water then is identified by the most abundant anion and cation. Commonly, the two most abundant anions and cations are stated. The chemical character also can be identified graphically from Piper diagrams and Stiff diagrams. 


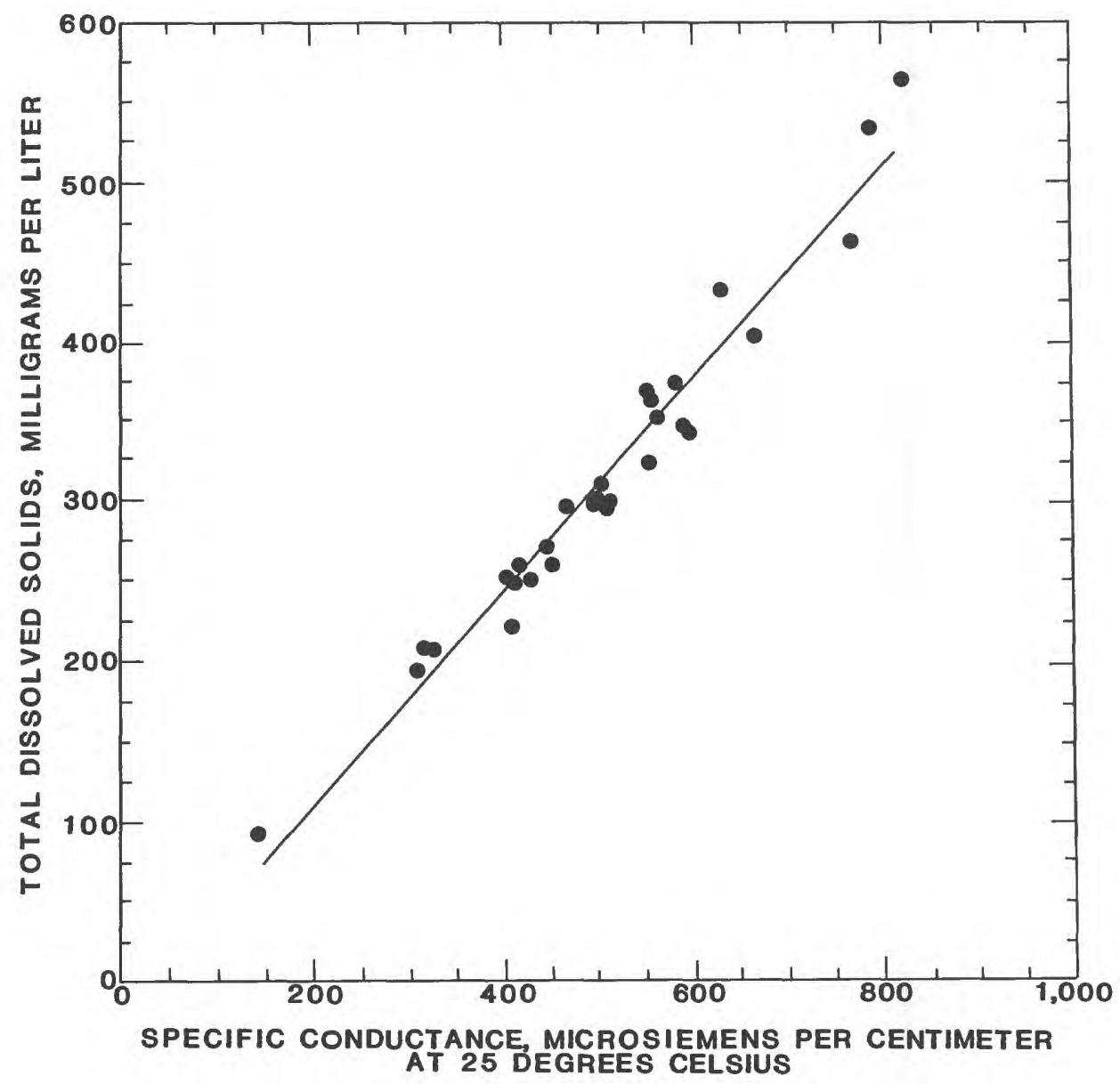

Figure 13.--Relation between specific conductance and total dissolved solids from 29 wells in Geauga County, Ohio. 
In Geauga County, the chemical character of the ground waters varies spatially and is related to the geologic material in which the well is completed (stratigraphic unit) and the position of the well within the regional and local flow systems. In addition, the manner in which the well was constructed (length of casing or open borehole) and the hydraulic conductivity of the geologic material in which the well is completed also may affect the chemical character of the ground water produced from the well.

Primary and Secondary Drinking-Water Regulations (USEPA, 1988a, 1988b, 1988c, 1988d, 1989a, 1989b) established by the U.S. Environmental Protection Agency (USEPA) set limitations on the quality of water that may be applied for specific uses. Maximum contaminant-level goals (MCLGs) are nonenforceable health-based standards set at a level to prevent known or anticipated adverse effects with an adequate margin of safety. Primary Drinking Water Regulations specify Maximum Contaminant Levels (MCLs) which are enforceable health based standards set as close to the MCLGs as feasible on the basis of water-treatment technologies and costs.

Nonenforceable Secondary Drinking-Water Regulations establish Secondary Maximum Contaminant Levels (SMCLs) for substances which affect the aesthetic quality of water by imparting taste and odor and by staining fixtures. MCLs and SMCLs relevant to the constituents listed in table 3 are included in the table.

Fecal streptococci bacteria are being used increasingly as a microbiological indicator of significant contamination of water because the normal habitat of these organisms is the intestines of humans and animals. The term "fecal streptococci" encompasses several varieties and groups of bacteria, some of which may not be of sanitary significance (Pagel and Hardy, 1980).

Bacteriological analyses were done according to Standard Methods for the Examination of Water and Wastewater (American Public Health Association, 1980); KF streptococcus agar was used for the enumeration of fecal streptococci. Brodsky and Schiemann (1976) investigated the recovery of fecal streptococci by KF agar and confirmed that 83 percent of the typical red-to-pink colonies recovered at 35 degrees Celsius were fecal streptococci. A similar study (Pagel and Hardy, 1980) confirmed 81 percent of typical colonies recovered were fecal streptococci, the remaining 19 percent being non-fecal streptococci.

Microbiological testing for fecal coliform and fecal streptococci bacteria was done on water from 38 wells within the study area. Water from 10 of the 38 wells tested (table 3 ) contained either fecal coliform or fecal streptococci bacteria or both. Concentrations of fecal bacteria ranged from $<1$ to $6 \mathrm{cols} / 100 \mathrm{~mL}$ (colonies per 100 milliliters). 
Table 2.--Concentrations of chemical constituents and physical properties of waters from the qlacial oeposits, pottsville Formation, Cuyahoga Group, Berea Sandstone, and Bediford Shase in Geaúa County, Onio

$\left[{ }^{\circ} \mathrm{C}\right.$, degrees Celsius; Fm.. Formation; mg/L, milligrams per liter; ug/L, micrograms per 1 iter; us/cm, microsiemens per centimeter at 25 degrees Celsius: mililiter; <, less than; -- data not available; MCL, Maximum contaminent level, SMCL, Secondary Maximum Contaminent Level]

\begin{tabular}{|c|c|c|c|c|c|c|c|c|c|}
\hline $\begin{array}{c}\text { Well } 1 \\
\text { number }\end{array}$ & $\begin{array}{c}\text { Aqui fer } \\
\text { name }\end{array}$ & Date & $\begin{array}{c}\text { Temper- } \\
\text { ature, } \\
\text { water } \\
\left({ }^{\circ} \mathrm{C}\right)\end{array}$ & $\begin{array}{c}\text { Temper- } \\
\text { ature, } \\
\text { air } \\
\left({ }^{\circ} \mathrm{C}\right)\end{array}$ & $\begin{array}{r}\text { Spe- } \\
\text { cific } \\
\text { con- } \\
\text { duc- } \\
\text { tance } \\
(u S / \mathrm{cm})\end{array}$ & $\begin{array}{l}\text { Oxygen } \\
\text { demand, } \\
\text { chem- } \\
\text { ical } \\
\text { (high } \\
\text { level) } \\
\text { (mg/L) }\end{array}$ & $\begin{array}{c}\mathrm{pH} \\
\text { (stand- } \\
\text { ard } \\
\text { units } \\
\text { pH) }\end{array}$ & $\begin{array}{l}\text { Alka- } \\
\text { linity } \\
(\mathrm{mg} / \mathrm{L} \text { as } \\
\left.\mathrm{CaCO}_{3}\right)\end{array}$ & $\begin{array}{l}\text { Nitro- } \\
\text { gen, } \\
\text { organic, } \\
\text { dis- } \\
\text { solved } \\
\text { (mg/L } \\
\text { as } N \text { ) }\end{array}$ \\
\hline $\begin{array}{l}\text { GE-92 } \\
G E-101 \\
\text { GE-108 } \\
G E-116 \\
G E-141\end{array}$ & Glacial deposits & $\begin{array}{l}04-24-86 \\
04-14-86 \\
05-01-86 \\
04-30-86 \\
05-01-86\end{array}$ & $\begin{array}{l}10.5 \\
10.0 \\
11.5 \\
10.5 \\
11.5\end{array}$ & $\begin{array}{r}7.5 \\
20.0 \\
21: 0 \\
16.0 \\
20.0\end{array}$ & $\begin{array}{l}625 \\
554 \\
328 \\
500 \\
493\end{array}$ & $\begin{array}{r}10 \\
18 \\
33 \\
<10 \\
<10\end{array}$ & $\begin{array}{l}7.60 \\
7.30 \\
8.10 \\
7.50 \\
7.50\end{array}$ & $\begin{array}{l}290 \\
224 \\
167 \\
250 \\
186\end{array}$ & $\begin{array}{l}0.08 \\
0.19 \\
0.13 \\
0.13 \\
--\end{array}$ \\
\hline $\begin{array}{l}\text { GE-165 } \\
\text { GE-174 } \\
\text { GE-175 }\end{array}$ & & $\begin{array}{l}05-01-86 \\
04-23-86 \\
04-23-86\end{array}$ & $\begin{array}{l}10.0 \\
11.0 \\
11.0\end{array}$ & $\begin{array}{l}22.0 \\
16.0 \\
13.5\end{array}$ & $\begin{array}{l}405 \\
551 \\
416\end{array}$ & $\begin{array}{r}<10 \\
<10 \\
10\end{array}$ & $\begin{array}{l}7.70 \\
7.30 \\
7.60\end{array}$ & $\begin{array}{l}171 \\
291 \\
217\end{array}$ & $\begin{array}{l}0.14 \\
0.13 \\
0.08\end{array}$ \\
\hline $\begin{array}{l}G E-39 \\
G E-60\end{array}$ & Pottsville $\mathrm{Em}$. & $\begin{array}{l}04-25-86 \\
04-29-86\end{array}$ & $\begin{array}{r}12.5 \\
-.-\end{array}$ & $\begin{array}{l}26.0 \\
25.0\end{array}$ & $\frac{690}{--}$ & $\begin{array}{l}<10 \\
<10\end{array}$ & $\begin{array}{l}6.90 \\
7.20\end{array}$ & $\begin{array}{l}164 \\
187\end{array}$ & $\begin{array}{l}0.24 \\
0.14\end{array}$ \\
\hline $\begin{array}{l}G E-68 \\
G E-69 \\
G E-73 \\
G E-89 \\
G E-109\end{array}$ & & $\begin{array}{l}04-24-86 \\
04-22-86 \\
04-15-86 \\
04-23-86 \\
04-22-86\end{array}$ & $\begin{array}{r}9.0 \\
12.5 \\
10.5 \\
11.5 \\
10.5\end{array}$ & $\begin{array}{r}26.0 \\
5.5 \\
12.0 \\
12.0 \\
2.0\end{array}$ & $\begin{array}{r}140 \\
2650 \\
560 \\
903 \\
463\end{array}$ & $\begin{array}{r}<10 \\
14 \\
12 \\
<10 \\
<10\end{array}$ & $\begin{array}{l}6.50 \\
6.90 \\
7.30 \\
7.10 \\
7.50\end{array}$ & $\begin{array}{r}26 \\
151 \\
242 \\
252 \\
215\end{array}$ & $\begin{array}{l}0.38 \\
0.19 \\
0.18 \\
0.23 \\
0.13\end{array}$ \\
\hline $\begin{array}{l}\mathrm{GE}-111 \\
\mathrm{GE}-112 \\
\mathrm{GE}-115 \\
\mathrm{GE}-151 \\
\mathrm{GE}-180\end{array}$ & & $\begin{array}{l}04-17-86 \\
04-17-86 \\
04-24-86 \\
04-29-86 \\
04-23-86\end{array}$ & $\begin{array}{r}10.0 \\
10.0 \\
11.5 \\
11.5\end{array}$ & $\begin{array}{l}13.5 \\
12.0 \\
19.0 \\
25.0 \\
14.5\end{array}$ & $\begin{array}{r}307 \\
729 \\
315 \\
369\end{array}$ & $\begin{array}{r}<10 \\
<10 \\
14 \\
<10 \\
<10\end{array}$ & $\begin{array}{l}7.10 \\
6.90 \\
6.60 \\
7.50 \\
5.10\end{array}$ & $\begin{array}{r}135 \\
220 \\
105 \\
174 \\
55\end{array}$ & $\begin{array}{l}0 . \overline{37} \\
0.16 \\
0.14 \\
0.37\end{array}$ \\
\hline $\begin{array}{l}\text { GE-202 } \\
\text { GE-48 } \\
G E-55 \\
G E-67 \\
G E-77\end{array}$ & Cuyahoga Group & $\begin{array}{l}04-15-86 \\
04-16-86 \\
04-17-86 \\
04-24-86 \\
04-22-86\end{array}$ & $\begin{array}{l}11.5 \\
10.0 \\
10.5 \\
10.5 \\
11.5\end{array}$ & $\begin{array}{r}7.0 \\
4.5 \\
14.0 \\
29.0 \\
0.5\end{array}$ & $\begin{array}{l}504 \\
442 \\
495 \\
402 \\
813\end{array}$ & $\begin{array}{r}<10 \\
12 \\
17 \\
17 \\
<10\end{array}$ & $\begin{array}{l}7.20 \\
7.90 \\
9.10 \\
8.50 \\
7.40\end{array}$ & $\begin{array}{l}254 \\
257 \\
229 \\
218 \\
376\end{array}$ & $\begin{array}{l}0.08 \\
0.17 \\
0.15 \\
0.06 \\
0.0\end{array}$ \\
\hline $\begin{array}{l}\text { GE-105 } \\
\text { GE-147 } \\
\text { GE-169 } \\
\text { GE-186 } \\
\text { GE-102 }\end{array}$ & Berea Sandstone & $\begin{array}{l}04-15-86 \\
04-30-86 \\
04-29-86 \\
04-15-86 \\
04-15-86\end{array}$ & $\begin{array}{l}11.0 \\
11.5 \\
11.0 \\
10.0 \\
11.0\end{array}$ & $\begin{array}{r}5.5 \\
23.0 \\
27.0 \\
12.0 \\
6.5\end{array}$ & $\begin{array}{l}978 \\
780 \\
450 \\
577 \\
594\end{array}$ & $\begin{array}{l}12 \\
15 \\
33 \\
14 \\
11\end{array}$ & $\begin{array}{l}8.20 \\
7.60 \\
8.20 \\
7.80 \\
7.30\end{array}$ & $\begin{array}{l}416 \\
270 \\
205 \\
327 \\
244\end{array}$ & $\begin{array}{l}0.16 \\
0.01 \\
0.05 \\
0.07 \\
0.17\end{array}$ \\
\hline $\begin{array}{l}\text { GE }-103 \\
\text { GE-104 } \\
G E-120 \\
G E-122 \\
G E-125\end{array}$ & & $\begin{array}{l}05-01-86 \\
05-01-86 \\
04-18-86 \\
04-21-86 \\
04-30-86\end{array}$ & $\begin{array}{l}11.5 \\
11.0 \\
10.0 \\
11.0 \\
11.0\end{array}$ & $\begin{array}{r}16.0 \\
18.0 \\
16.5 \\
8.5 \\
20.0\end{array}$ & $\begin{array}{r}550 \\
510 \\
426 \\
1,450 \\
660\end{array}$ & $\begin{array}{r}10 \\
<10 \\
10 \\
34 \\
<10\end{array}$ & $\begin{array}{l}7.40 \\
7.90 \\
7.90 \\
7.10 \\
7.20\end{array}$ & $\begin{array}{l}284 \\
257 \\
219 \\
335 \\
277\end{array}$ & $\begin{array}{l}0.14 \\
0.19 \\
0.09 \\
0.0 \\
0\end{array}$ \\
\hline GE-159 & & $04-30-86$ & 12.5 & 20.0 & 760 & 21 & -- & 335 & 0.08 \\
\hline $\begin{array}{l}G E-148 \\
G E-44\end{array}$ & Bedford Shale & $\begin{array}{l}04-16-86 \\
04-17-86\end{array}$ & $\begin{array}{l}10.5 \\
10.5\end{array}$ & $\begin{array}{r}3.5 \\
10.0\end{array}$ & $\begin{array}{l}263 \\
489\end{array}$ & $\begin{array}{l}11 \\
18\end{array}$ & $\begin{array}{l}7.50 \\
8.20\end{array}$ & $\begin{array}{l}119 \\
184\end{array}$ & $\begin{array}{l}0.07 \\
0.08\end{array}$ \\
\hline $\begin{array}{l}\text { MCL } \\
\text { SMCL }\end{array}$ & & & & & & & $6.5-$ & 8.5 & \\
\hline
\end{tabular}


Table 3.--Concentrations of chemical constituents and physical properties of waters from the olacial deposits, Pottsville Formation. Cuyahoga Group, Eerea Sandstone, and Bedford Shale in Geauga County, ohio--continued

\begin{tabular}{|c|c|c|c|c|c|c|c|c|c|}
\hline $\begin{array}{c}\text { Wel } 1 \\
\text { number }\end{array}$ & $\begin{array}{c}\text { Aquifer } \\
\text { name }\end{array}$ & $\begin{array}{l}\text { Nitro- } \\
\text { gen, } \\
\text { ammonia, } \\
\text { dis- } \\
\text { solved } \\
\text { (mg/L } \\
\text { as } N \text { ) }\end{array}$ & $\begin{array}{c}\text { Nitro- } \\
\text { gen, } \\
\mathrm{NO}_{2}+\mathrm{NO}_{3} \\
\text { dis- } \\
\text { solved } \\
\text { ( } \mathrm{mg} / \mathrm{L} \\
\text { as } \mathrm{N} \text { ) }\end{array}$ & $\begin{array}{l}\text { Phos- } \\
\text { phorous, } \\
\text { ortho, } \\
\text { dis- } \\
\text { solved } \\
\text { (mg/L } \\
\text { as P) }\end{array}$ & $\begin{array}{c}\text { Carbon, } \\
\text { organic, } \\
\text { total } \\
\text { (mg/L } \\
\text { as }(\text { ) }\end{array}$ & $\begin{array}{l}\text { Harō- } \\
\text { ness } \\
\text { total } \\
\text { (mg/L } \\
\text { as } \\
\left.\mathrm{CaCO}_{3}\right)\end{array}$ & $\begin{array}{c}\text { Hard- } \\
\text { ness, } \\
\text { noncarb- } \\
\text { onate } \\
\left(\mathrm{mg}_{\mathrm{L}} \text { as }\right. \\
\left.\mathrm{CaCO}_{3}\right)\end{array}$ & $\begin{array}{l}\text { Calcium, } \\
\text { - dis- } \\
\text { solved } \\
\text { s (mg/L } \\
\text { as Ca) }\end{array}$ & $\begin{array}{l}\text { Niagne- } \\
\text { sium, } \\
\text { dis- } \\
\text { solved } \\
\text { (mg/L } \\
\text { as } \mathrm{Mg} \text { ) }\end{array}$ \\
\hline $\begin{array}{l}\mathrm{GE}-92 \\
\mathrm{GE}-101 \\
\mathrm{GE}-108 \\
\mathrm{GE}-116 \\
\mathrm{GE}-141\end{array}$ & Glacial óposits & $\begin{array}{l}0.120 \\
0.110 \\
0.270 \\
0.070 \\
0.050\end{array}$ & $\begin{array}{l}<0.100 \\
<0.100 \\
<0.100 \\
<0.100 \\
<0.100\end{array}$ & $\begin{array}{l}<0.010 \\
<0.010 \\
<0.010 \\
<0.010 \\
<0.010\end{array}$ & $\begin{array}{r}2.8 \\
0.4 \\
<0.1 \\
1.1 \\
0.1\end{array}$ & $\begin{array}{r}370 \\
290 \\
9 \\
170 \\
230\end{array}$ & $\begin{array}{r}82 \\
67 \\
0 \\
0 \\
48\end{array}$ & $\begin{array}{l}89 \\
80 \\
1.8 \\
44 \\
67\end{array}$ & $\begin{array}{l}36 \\
22 \\
1.2 \\
14 \\
16\end{array}$ \\
\hline $\begin{array}{l}\mathrm{GE}-165 \\
\mathrm{GE}-174 \\
\mathrm{GE}-175 \\
\mathrm{GE}-39 \\
\mathrm{GE}-60\end{array}$ & Pottsville Fm. & $\begin{array}{l}0.060 \\
0.170 \\
0.120 \\
0.060 \\
0.060\end{array}$ & $\begin{array}{c}<0.100 \\
<0.100 \\
<0.100 \\
1.30 \\
6.00\end{array}$ & $\begin{array}{l}<0.010 \\
<0.010 \\
<0.010 \\
<0.010 \\
<0.010\end{array}$ & $\begin{array}{c}2.8 \\
0.9 \\
0.9 \\
1.5 \\
<0.1\end{array}$ & $\begin{array}{l}200 \\
320 \\
230 \\
320 \\
280\end{array}$ & $\begin{array}{r}29 \\
26 \\
13 \\
160 \\
97\end{array}$ & $\begin{array}{l}60 \\
84 \\
64 \\
95 \\
79\end{array}$ & $\begin{array}{l}12 \\
26 \\
17 \\
21 \\
21\end{array}$ \\
\hline $\begin{array}{l}\mathrm{GE}-68 \\
\mathrm{GE}-69 \\
\mathrm{GE}-73 \\
\mathrm{GE}-89 \\
\mathrm{GE}-109\end{array}$ & & $\begin{array}{l}0.020 \\
0.110 \\
0.020 \\
0.070 \\
0.070\end{array}$ & $\begin{array}{l}2.90 \\
0.540 \\
0.220 \\
0.660 \\
<0.100\end{array}$ & $\begin{array}{l}<0.010 \\
<0.010 \\
<0.010 \\
<0.010 \\
<0.010\end{array}$ & $\begin{array}{l}0.6 \\
1.3 \\
0.8 \\
0.7 \\
1.8\end{array}$ & $\begin{array}{r}52 \\
700 \\
300 \\
460 \\
260\end{array}$ & $\begin{array}{r}26 \\
550 \\
59 \\
210 \\
47\end{array}$ & $\begin{array}{r}15 \\
210 \\
79 \\
120 \\
70\end{array}$ & $\begin{array}{l}3.4 \\
42 \\
25 \\
40 \\
21\end{array}$ \\
\hline $\begin{array}{l}\mathrm{GE}-111 \\
\mathrm{GE}-112 \\
\mathrm{GE}-115 \\
\mathrm{GE}-151 \\
\mathrm{GE}-1 \mathrm{~B} 0\end{array}$ & & $\begin{array}{r}<0.010 \\
0.030 \\
0.040 \\
0.060 \\
0.030\end{array}$ & $\begin{array}{c}1.40 \\
2.00 \\
<0.100 \\
<0.100 \\
2.20\end{array}$ & $\begin{array}{l}<0.010 \\
<0.010 \\
<0.010 \\
<0.010 \\
<0.010\end{array}$ & $\begin{array}{l}0.5 \\
0.6 \\
0.7 \\
0.4 \\
1.0\end{array}$ & $\begin{array}{l}160 \\
350 \\
160 \\
210 \\
110\end{array}$ & $\begin{array}{r}29 \\
130 \\
52 \\
41 \\
58\end{array}$ & $\begin{array}{l}49 \\
98 \\
43 \\
58 \\
33\end{array}$ & $\begin{array}{l}10 \\
26 \\
12 \\
17 \\
7.4\end{array}$ \\
\hline $\begin{array}{l}\mathrm{GE}-202 \\
\mathrm{GE}-48 \\
\mathrm{GE}-55 \\
\mathrm{GE}-67 \\
\mathrm{GE}-77\end{array}$ & Cuyahoga Group & $\begin{array}{l}0.120 \\
0.230 \\
0.350 \\
0.340 \\
1.10\end{array}$ & $\begin{array}{l}<0.100 \\
<0.100 \\
<0.100 \\
<0.100 \\
<0.100\end{array}$ & $\begin{array}{r}<0.010 \\
0.010 \\
0.050 \\
<0.010 \\
<0.010\end{array}$ & $\begin{array}{l}0.5 \\
1.4 \\
1.2 \\
0.8 \\
1.1\end{array}$ & $\begin{array}{r}270 \\
190 \\
5 \\
42 \\
400\end{array}$ & $\begin{array}{r}19 \\
0 \\
0 \\
0 \\
23\end{array}$ & $\begin{array}{l}71 \\
41 \\
1.3 \\
11 \\
92\end{array}$ & $\begin{array}{l}23 \\
21 \\
0.32 \\
3.4 \\
41\end{array}$ \\
\hline $\begin{array}{l}\text { GE-105 } \\
\mathrm{GE}-147 \\
\mathrm{GE}-169 \\
\mathrm{GE}-186 \\
\mathrm{GE}-102\end{array}$ & Eerea Sandstone & $\begin{array}{l}0.740 \\
0.890 \\
0.550 \\
0.730 \\
0.030\end{array}$ & $\begin{array}{l}<0.100 \\
<0.100 \\
<0.100 \\
<0.100 \\
<0.100\end{array}$ & $\begin{array}{r}0.020 \\
<0.010 \\
<0.010 \\
0.030 \\
<0.010\end{array}$ & $\begin{array}{r}9.0 \\
<0.1 \\
<0.1 \\
0.5 \\
0.8\end{array}$ & $\begin{array}{r}28 \\
260 \\
69 \\
50 \\
300\end{array}$ & $\begin{array}{r}0 \\
0 \\
0 \\
0 \\
61\end{array}$ & $\begin{array}{l}7.2 \\
63 \\
17 \\
13 \\
84\end{array}$ & $\begin{array}{c}2.3 \\
24 \\
6.3 \\
4.1 \\
23\end{array}$ \\
\hline $\begin{array}{l}\text { GE-103 } \\
\text { GE-104 } \\
G E-120 \\
G E-122 \\
G E-126\end{array}$ & & $\begin{array}{l}0.360 \\
0.710 \\
0.210 \\
1.40 \\
0.170\end{array}$ & $\begin{array}{r}<0.100 \\
<0.100 \\
0.470 \\
<0.100 \\
<0.100\end{array}$ & $\begin{array}{r}<0.010 \\
0.010 \\
0.010 \\
<0.010 \\
<0.010\end{array}$ & $\begin{array}{r}<0.1 \\
0.7 \\
0.7 \\
1.3 \\
<0.1\end{array}$ & $\begin{array}{r}240 \\
140 \\
72 \\
790 \\
310\end{array}$ & $\begin{array}{r}0 \\
0 \\
0 \\
450 \\
38\end{array}$ & $\begin{array}{r}56 \\
34 \\
18 \\
180 \\
88\end{array}$ & $\begin{array}{l}25 \\
14 \\
6.4 \\
82 \\
23\end{array}$ \\
\hline$G E-159$ & & 0.420 & $<0.100$ & 0.020 & 3.1 & 6 & 0 & 1.8 & 0.46 \\
\hline $\begin{array}{l}\mathrm{GE}-148 \\
\mathrm{GE}-44\end{array}$ & Beöford Shale & $\begin{array}{l}0.130 \\
0.420\end{array}$ & $\begin{array}{l}<0.100 \\
<0.100\end{array}$ & $\begin{array}{r}<0.010 \\
0.030\end{array}$ & $\begin{array}{l}0.4 \\
0.3\end{array}$ & $\begin{array}{r}130 \\
5\end{array}$ & $\begin{array}{l}7 \\
0\end{array}$ & $\begin{array}{l}34 \\
1.2\end{array}$ & $\begin{array}{l}10 \\
0.33\end{array}$ \\
\hline $\begin{array}{l}\text { MCL } \\
\text { SMCL }\end{array}$ & & & 10 & & & & & & \\
\hline
\end{tabular}



Table 3.--Concentrations of chemical constituents, and physical properties of waters from the qlacial deposits,
pottsville Formation, Cuyahoga Group, Berea Sandstone, and Bedford Shale in Geauga County, Ohio--Continued

\begin{tabular}{|c|c|c|c|c|c|c|c|c|c|}
\hline $\begin{array}{l}\text { well } 1 \\
\text { number }\end{array}$ & $\begin{array}{c}\text { Aquifer } \\
\text { name }\end{array}$ & $\begin{array}{l}\text { Sodium, } \\
\text { dis- } \\
\text { solved } \\
\text { (mg/L } \\
\text { as Na) }\end{array}$ & $\begin{array}{l}\text { Potas- } \\
\text { sium, } \\
\text { dis- } \\
\text { solved } \\
\text { (mg/L } \\
\text { as K) }\end{array}$ & $\begin{array}{l}\text { Chlo- } \\
\text { ride, } \\
\text { dis- } \\
\text { solved } \\
\text { (mg/L } \\
\text { as C1) }\end{array}$ & $\begin{array}{c}\text { Sulfate } \\
\text { dis- } \\
\text { solved } \\
\text { (mg/L } \\
\text { as } \mathrm{SO}_{4} \text { ) }\end{array}$ & $\begin{array}{l}\text { Fluo- } \\
\text { ride, } \\
\text { dis- } \\
\text { solved } \\
\text { (mg/L } \\
\text { as F) }\end{array}$ & $\begin{array}{l}\text { Silica, } \\
\text { dis- } \\
\text { solved } \\
\text { (mg/L } \\
\text { as } \\
\mathrm{SiO}_{2} \text { ) }\end{array}$ & $\begin{array}{l}\text { Barium, } \\
\text { dis- } \\
\text { solved } \\
\text { ( } \mu \mathrm{g} / \mathrm{L} \\
\text { as Ba) }\end{array}$ & $\begin{array}{c}\text { Iron, } \\
\text { dis- } \\
\text { solved } \\
\text { (ug/L } \\
\text { as Fe) }\end{array}$ \\
\hline $\begin{array}{l}\text { GE-92 } \\
G E-101 \\
G E-108 \\
G E-116 \\
G E-141\end{array}$ & Glacial deposits & $\begin{array}{l}9.0 \\
5.8 \\
76 \\
48 \\
6.0\end{array}$ & $\begin{array}{l}1.5 \\
1.6 \\
1.6 \\
1.3 \\
1.0\end{array}$ & $\begin{array}{l}2.1 \\
4.5 \\
0.80 \\
1.3 \\
21\end{array}$ & $\begin{array}{r}100 \\
73 \\
13 \\
41 \\
53\end{array}$ & $\begin{array}{r}0.20 \\
0.20 \\
0.30 \\
<0.10 \\
0.10\end{array}$ & $\begin{array}{l}16 \\
14 \\
12 \\
14 \\
10\end{array}$ & $\begin{array}{r}<100 \\
100 \\
<100 \\
<100 \\
200\end{array}$ & $\begin{array}{r}2,800 \\
1,300 \\
72 \\
790 \\
1,000\end{array}$ \\
\hline $\begin{array}{l}\text { GE }-165 \\
\text { GE }-174 \\
\text { GE }-175 \\
\text { GE }-39 \\
\text { GE-60 }\end{array}$ & Pottsville Fm. & $\begin{array}{c}4.7 \\
8.4 \\
7.6 \\
32 \\
6.1\end{array}$ & $\begin{array}{l}0.80 \\
1.4 \\
1.6 \\
2.4 \\
1.0\end{array}$ & $\begin{array}{c}11 \\
1.5 \\
1.3 \\
120 \\
20\end{array}$ & $\begin{array}{l}24 \\
58 \\
19 \\
63 \\
58\end{array}$ & $\begin{array}{r}<0.10 \\
0.20 \\
0.20 \\
0.10 \\
0.20\end{array}$ & $\begin{array}{l}10 \\
20 \\
16 \\
8.6 \\
10\end{array}$ & $\begin{array}{r}100 \\
100 \\
100 \\
<100\end{array}$ & $\begin{array}{r}1,800 \\
1,400 \\
780 \\
11 \\
10\end{array}$ \\
\hline $\begin{array}{l}G E-68 \\
G E-69 \\
G E-73 \\
G E-89 \\
G E-109\end{array}$ & & $\begin{array}{c}7.1 \\
310 \\
4.8 \\
14 \\
4.5\end{array}$ & $\begin{array}{l}1.5 \\
1.8 \\
1.4 \\
1.8 \\
1.0\end{array}$ & $\begin{array}{c}11 \\
780 \\
3.1 \\
130 \\
2.6\end{array}$ & $\begin{array}{r}15 \\
120 \\
68 \\
74 \\
58\end{array}$ & $\begin{array}{r}<0.10 \\
0.10 \\
0.20 \\
0.20 \\
0.20\end{array}$ & $\begin{array}{l}9.1 \\
9.1 \\
9.6 \\
13 \\
11\end{array}$ & $\begin{array}{l}<100 \\
200 \\
100 \\
100 \\
100\end{array}$ & $\begin{array}{r}79 \\
70 \\
390 \\
10 \\
370\end{array}$ \\
\hline $\begin{array}{l}\text { GE-111 } \\
G E-112 \\
G E-115 \\
G E-151 \\
G E-180\end{array}$ & & $\begin{array}{c}3.3 \\
17 \\
5.3 \\
3.4 \\
30\end{array}$ & $\begin{array}{l}0.60 \\
1.3 \\
1.3 \\
1.0 \\
1.5\end{array}$ & $\begin{array}{c}2.7 \\
71 \\
4.5 \\
2.9 \\
63\end{array}$ & $\begin{array}{l}19 \\
61 \\
69 \\
49 \\
29\end{array}$ & $\begin{array}{r}0.20 \\
0.10 \\
<0.10 \\
0.30 \\
<0.10\end{array}$ & $\begin{array}{l}8.3 \\
8.2 \\
9.3 \\
10 \\
10\end{array}$ & $\begin{array}{r}200 \\
200 \\
<100 \\
<100 \\
<100\end{array}$ & $\begin{array}{r}11 \\
33 \\
4,200 \\
470 \\
26\end{array}$ \\
\hline $\begin{array}{l}\mathrm{GE}-202 \\
\mathrm{GE}-48 \\
\mathrm{GE}-55 \\
\mathrm{GE}-67 \\
\mathrm{GE}-77\end{array}$ & Cuyahoga Group & $\begin{array}{c}8.0 \\
28 \\
120 \\
84 \\
49\end{array}$ & $\begin{array}{l}1.5 \\
2.5 \\
1.2 \\
2.4 \\
6.4\end{array}$ & $\begin{array}{l}1.6 \\
2.6 \\
25 \\
10 \\
1.9\end{array}$ & $\begin{array}{c}30 \\
4.0 \\
5.5 \\
0.70 \\
120\end{array}$ & $\begin{array}{l}0.40 \\
0.40 \\
0.90 \\
1.2 \\
0.20\end{array}$ & $\begin{array}{c}16 \\
17 \\
7.9 \\
7.9 \\
19\end{array}$ & $\begin{array}{r}200 \\
300 \\
100 \\
500 \\
<100\end{array}$ & $\begin{array}{r}590 \\
1,100 \\
27 \\
160 \\
1,400\end{array}$ \\
\hline $\begin{array}{l}\text { GE-105 } \\
G E-147 \\
G E-169 \\
G E-186 \\
G E-102\end{array}$ & Berea Sanỏstone & $\begin{array}{r}220 \\
81 \\
73 \\
120 \\
11\end{array}$ & $\begin{array}{l}2.3 \\
5.6 \\
3.2 \\
2.6 \\
1.3\end{array}$ & $\begin{array}{c}120 \\
6.3 \\
26 \\
4.2 \\
29\end{array}$ & $\begin{array}{c}5.0 \\
170 \\
3.0 \\
14 \\
38\end{array}$ & $\begin{array}{l}1.1 \\
0.20 \\
0.50 \\
0.60 \\
0.20\end{array}$ & $\begin{array}{c}7.2 \\
14 \\
9.8 \\
9.9 \\
9.5\end{array}$ & $\begin{array}{r}900 \\
<100 \\
900 \\
200 \\
<100\end{array}$ & $\begin{array}{r}100 \\
770 \\
130 \\
20 \\
10\end{array}$ \\
\hline $\begin{array}{l}G E-103 \\
G E-104 \\
G E-120 \\
G E-122 \\
G E-126\end{array}$ & & $\begin{array}{l}26 \\
57 \\
67 \\
86 \\
14\end{array}$ & $\begin{array}{l}2.9 \\
2.7 \\
2.3 \\
4.5 \\
1.8\end{array}$ & $\begin{array}{l}1.2 \\
5.1 \\
3.2 \\
6.8 \\
16\end{array}$ & $\begin{array}{r}21 \\
17 \\
12 \\
660 \\
73\end{array}$ & $\begin{array}{l}0.30 \\
0.30 \\
0.70 \\
0.10 \\
0.20\end{array}$ & $\begin{array}{l}15 \\
12 \\
11 \\
13 \\
15\end{array}$ & $\begin{array}{r}100 \\
200 \\
500 \\
<100 \\
100\end{array}$ & $\begin{array}{r}840 \\
390 \\
13 \\
1,400 \\
660\end{array}$ \\
\hline$G E-159$ & & 190 & 1.1 & 53 & 0.90 & 0.70 & 7.3 & $<100$ & 17 \\
\hline $\begin{array}{l}G E-148 \\
G E-44\end{array}$ & Bedford shale & $110^{7.9}$ & $\begin{array}{l}1.1 \\
1.1\end{array}$ & $52^{3.2}$ & $\begin{array}{l}18 \\
4.2\end{array}$ & $\begin{array}{l}0.20 \\
1.1\end{array}$ & $\begin{array}{l}14 \\
7.5\end{array}$ & $\begin{array}{r}<100 \\
900\end{array}$ & $\begin{array}{r}310 \\
52\end{array}$ \\
\hline $\begin{array}{l}\text { NCL } \\
\text { SMCL }\end{array}$ & & & & 250 & 250 & 2.4 & & 1,000 & 300 \\
\hline
\end{tabular}


Table 3.--Concentrations of chemicai constituents and physical properties of waters from the glacial deposits. Pottsvil le Formation, Cuyahoga Group. Berea Sandstone, and Bedford Shale in Geauga county Onio--Continued

\begin{tabular}{|c|c|c|c|c|c|c|c|c|c|}
\hline $\begin{array}{l}\text { Wel I } \\
\text { number }\end{array}$ & $\begin{array}{c}\text { Aqui fer } \\
\text { name }\end{array}$ & $\begin{array}{c}\text { Manga- } \\
\text { nese, } \\
\text { dis- } \\
\text { solved } \\
\text { (ug/L } \\
\text { as !In) }\end{array}$ & $\begin{array}{c}\text { Stron- } \\
\text { tium, } \\
\text { dis- } \\
\text { solved } \\
\text { (ug/L } \\
\text { as St) }\end{array}$ & $\begin{array}{l}\text { Coli- } \\
\text { form, } \\
\text { fecai } \\
\text { (cols./ } \\
100 \mathrm{~mL} \text { ) }\end{array}$ & $\begin{array}{c}\text { Strep- } \\
\text { tococci } \\
\text { fecal } \\
\text { (cols.' } \\
100 \mathrm{~mL} \text { ) }\end{array}$ & $\begin{array}{c}\text { Enenols, } \\
\text { total } \\
\text { (ug/L) }\end{array}$ & $\begin{array}{c}\text { Methy- } \\
\text { lene } \\
\text { blue } \\
\text { active } \\
\text { sub- } \\
\text { stance } \\
\text { (mg/L) }\end{array}$ & $\begin{array}{c}\text { Solids, } \\
\text { residue } \\
\text { at } \\
180^{\circ} \mathrm{C}, \\
\text { dis- } \\
\text { solved } \\
(\mathrm{mg} / \mathrm{L})\end{array}$ & $\begin{array}{l}\text { Bromide, } \\
\text { dis- } \\
\text { solved } \\
\text { (mg/I } \\
\text { as Br) }\end{array}$ \\
\hline$G E-92$ & Glacial deposits & 96 & 500 & $<1$ & - & $<1$ & 0.03 & 430 & 0.15 \\
\hline $\begin{array}{l}G E-101 \\
G E-108 \\
G E-116 \\
G E-141\end{array}$ & & $\begin{array}{r}89 \\
3 \\
91 \\
180\end{array}$ & $\begin{array}{r}190 \\
29 \\
160 \\
95\end{array}$ & $\begin{array}{l}<1 \\
<1 \\
<1 \\
<1\end{array}$ & $\frac{{ }_{\mathrm{X} 3} 3}{--}$ & $\begin{array}{l}1 \\
<1 \\
<1 \\
1\end{array}$ & $\begin{array}{l}0.04 \\
0.04 \\
0.04 \\
0.04\end{array}$ & $\begin{array}{l}360 \\
207 \\
308 \\
299\end{array}$ & $\begin{array}{l}0.077 \\
0.026 \\
0.034 \\
0.049\end{array}$ \\
\hline $\begin{array}{l}\text { GE-1 } 65 \\
\text { GE }-174 \\
\text { GE-1 } 75 \\
\text { GE-39 } \\
\text { GE-60 }\end{array}$ & Pottsville Fm. & $\begin{array}{r}180 \\
170 \\
200 \\
<1 \\
<1\end{array}$ & $\begin{array}{l}78 \\
200 \\
180 \\
150 \\
110\end{array}$ & $\begin{array}{l}<1 \\
<1 \\
<1 \\
<1 \\
<1\end{array}$ & $\begin{array}{l}-- \\
<1 \\
<1 \\
<1 \\
<1\end{array}$ & $\begin{array}{l}<1 \\
<1 \\
<1 \\
<1 \\
<1\end{array}$ & $\begin{array}{l}0.04 \\
0.04 \\
0.03 \\
0.07 \\
0.09\end{array}$ & $\begin{array}{l}220 \\
365 \\
256 \\
455 \\
343\end{array}$ & $\begin{array}{l}0.16 \\
0.022 \\
0.021 \\
0.26 \\
0.084\end{array}$ \\
\hline $\begin{array}{l}\text { GE-68 } \\
G E-69 \\
G E-73 \\
G E-89 \\
G E-109\end{array}$ & & $\begin{array}{r}13 \\
<10 \\
5 \\
<1 \\
120\end{array}$ & $\begin{array}{l}51 \\
400 \\
150 \\
240 \\
280\end{array}$ & $\begin{array}{l}<1 \\
<1 \\
<1 \\
<1 \\
<1\end{array}$ & $\begin{array}{l}-- \\
<1 \\
K 1 \\
<1 \\
<1\end{array}$ & $\begin{array}{l}<1 \\
<1 \\
2 \\
<1 \\
<1\end{array}$ & $\begin{array}{l}0.06 \\
0.13 \\
0.04 \\
0.07 \\
0.03\end{array}$ & $\begin{array}{r}89 \\
1670 \\
349 \\
540 \\
293\end{array}$ & $\begin{array}{l}0.013 \\
0.21 \\
0.031 \\
0.18 \\
0.022\end{array}$ \\
\hline $\begin{array}{l}\text { GE-111 } \\
\text { GE-112 } \\
\text { GE-115 } \\
\text { GE-151 } \\
\text { GE-180 }\end{array}$ & & $\begin{array}{r}1 \\
2 \\
810 \\
90 \\
2\end{array}$ & $\begin{array}{r}78 \\
150 \\
64 \\
93 \\
65\end{array}$ & $\begin{array}{l}<1 \\
<1 \\
<1 \\
<1 \\
<1\end{array}$ & $\begin{array}{l}<1 \\
k 5 \\
-<1 \\
<1\end{array}$ & $\begin{array}{l}<1 \\
1 \\
1 \\
<1 \\
<1\end{array}$ & $\begin{array}{l}0.05 \\
0.05 \\
0.03 \\
0.03 \\
0.07\end{array}$ & $\begin{array}{l}195 \\
443 \\
207 \\
248 \\
225\end{array}$ & $\begin{array}{l}0.033 \\
0.11 \\
0.021 \\
0.049 \\
0.12\end{array}$ \\
\hline $\begin{array}{l}\mathrm{GE}-202 \\
\mathrm{GE}-48 \\
\mathrm{GE}-55 \\
\mathrm{GE}-67 \\
\mathrm{GE}-77\end{array}$ & Cuyahoga Group & $\begin{array}{r}96 \\
34 \\
2 \\
11 \\
27\end{array}$ & $\begin{array}{r}240 \\
360 \\
30 \\
97 \\
1\end{array}$ & $\begin{array}{l}<1 \\
<1 \\
<1 \\
<1 \\
<1\end{array}$ & $\begin{array}{l}\times 3 \\
\times 1 \\
\times 1 \\
<1\end{array}$ & $\begin{array}{r}2 \\
1 \\
<1 \\
1 \\
<1\end{array}$ & $\begin{array}{l}0.04 \\
0.04 \\
0.04 \\
0.04 \\
0.04\end{array}$ & $\begin{array}{l}297 \\
255 \\
306 \\
262 \\
544\end{array}$ & $\begin{array}{l}0.025 \\
0.079 \\
0.23 \\
0.21 \\
0.048\end{array}$ \\
\hline $\begin{array}{l}\mathrm{GE}-105 \\
\mathrm{GE}-147 \\
\mathrm{GE}-169\end{array}$ & & $\begin{array}{r}8 \\
15 \\
7\end{array}$ & $\begin{array}{l}190 \\
790 \\
200\end{array}$ & $\begin{array}{l}<1 \\
<1 \\
<1\end{array}$ & $\begin{array}{l}\times 2 \\
<1 \\
<1\end{array}$ & $\begin{array}{l}<1 \\
<1 \\
<1\end{array}$ & $\begin{array}{l}0.06 \\
0.03 \\
0.04\end{array}$ & $\begin{array}{l}578 \\
524 \\
268\end{array}$ & $\begin{array}{l}1.1 \\
0.10 \\
0.25\end{array}$ \\
\hline $\begin{array}{l}\text { GE-186 } \\
G E-102\end{array}$ & Berea Sandstone & 39 & $\begin{array}{l}240 \\
110\end{array}$ & $\begin{array}{r}1_{\mathrm{K} 1} \\
<1\end{array}$ & $\begin{array}{l}K 3 \\
K 1\end{array}$ & $\begin{array}{l}4 \\
1\end{array}$ & $\begin{array}{l}0.04 \\
0.04\end{array}$ & $\begin{array}{l}367 \\
351\end{array}$ & $\begin{array}{l}0.046 \\
0.084\end{array}$ \\
\hline $\begin{array}{l}\text { GE-103 } \\
\text { GE-104 } \\
G E-120 \\
G E-122\end{array}$ & & $\begin{array}{r}170 \\
9 \\
5 \\
70\end{array}$ & $\begin{array}{r}890 \\
590 \\
260 \\
1300\end{array}$ & $\begin{array}{l}<1 \\
<1 \\
<1 \\
<1\end{array}$ & $\begin{array}{l}-- \\
\overline{1} \\
1\end{array}$ & $\begin{array}{r}<1 \\
<1 \\
2 \\
<1\end{array}$ & $\begin{array}{l}0.03 \\
0.03 \\
0.04 \\
0.03\end{array}$ & $\begin{array}{r}327 \\
301 \\
238 \\
1250\end{array}$ & $\begin{array}{l}0.042 \\
0.079 \\
0.051 \\
0.048\end{array}$ \\
\hline GE-126 & & 170 & 190 & $<1$ & $<1$ & 4 & 0.04 & 411 & 0.060 \\
\hline GE-159 & & 3 & 34 & $<1$ & $<1$ & $<1$ & 0.05 & 454 & 0.34 \\
\hline GE- 148 & Bedford Shale & 240 & 94 & $<1$ & $<1$ & 4 & 0.03 & 165 & 0.033 \\
\hline $\mathrm{GE}-44$ & & 3 & 26 & $<1$ & K2 & $<1$ & 0.06 & 294 & 0.37 \\
\hline $\begin{array}{l}\text { MCL } \\
\text { SMCL }\end{array}$ & & 50 & & 1 & 1 & & 0.50 & 500 & \\
\hline
\end{tabular}

$\mathrm{I}_{\mathrm{K}}$ Estimated count based on nonideal colony count. 


\section{Stratigraphic Variations}

\section{Glacial deposits}

Eight wells completed in the glacial deposits were sampled and analyzed for water chemistry (table 3). Figure 5 shows the locations of these wells. As shown on figure 14, the chemical character of the water from five of these wells (GE-101, GE-141, GE-165, GE-174, and GE-175) is a calcium bicarbonate-type, whereas that from one well (GE-92) is a calcium magnesium bicarbonate-type. The waters from all eight wells are bicarbonate-type water; however, two of the waters differ in their dominant cation. The water from well GE-116 has a mixed-cation character having nearly equal concentrations of calcium and sodium, whereas the water in well GE-108 is a sodium-type water.

The TDS concentration of all these waters is low (table 3), indicating that they are not highly mineralized. The total hardness of these waters is highly variable (table 3). Table 4 lists summary statistics of TDS and total hardness data for these waters.

None of these waters exceeds USEPA, MCLs or SMCLs for nitrate, chloride, sulfate, fluoride, barium, methylene-blue active substances (foaming agents), or TDS. Many of these waters (GE-92, GE-101, GE-116, GE-141, GE-65, GE-174, GE-175) exceed USEPA SMCLs for iron and manganese which are nonhealth-based.

\section{Pottsville Formation}

The waters from 13 wells completed in the Pottsville Formation were sampled and analyzed (fig. 5, table 3). The waters from six wells (GE-60, GE-73, GE-109, GE-111, GE-151, and GE-202) are of the calcium bicarbonate-type, whereas the waters from the remaining seven wells differ considerably in chemical character (fig. 15). The waters from wells GE-112 and GE-89 are calcium bicarbonate chloride-type, whereas the water from well GE-115 is a calcium bicarbonate sulfate-type. The waters from wells GE-39, GE-89, and GE-180 are bicarbonate chloride-types in terms of anion character, but range from calcium to calcium magnesium to calcium sodium-types, respectively, in terms of cation character. The water from well GE-68 is a calcium sodium, and mixed aniontype.

Well GE-69 is located at a State highway garage and has been contaminated by road salt. As a result, it is highly mineralized and has a TDS concentration of $1,670 \mathrm{mg} / \mathrm{L}$ (table 3). It is a calcium sodium-chloride water reflecting the dissolution of sodium chloride and calcium chloride road salts. Wells GE-39, GE-89, GE-112, and GE-180 also appear to be affected by road salt, although the TDS content of these waters (table 3) is less than $600 \mathrm{mg} / \mathrm{L}$. The general straight-line trend of points for these waters extending to the point for well GE-69 on the cation and anion triangles of figure 15 supports the theory of runoff from a road-salt source mixing with natural ground waters. 


\section{EXPLANATION}

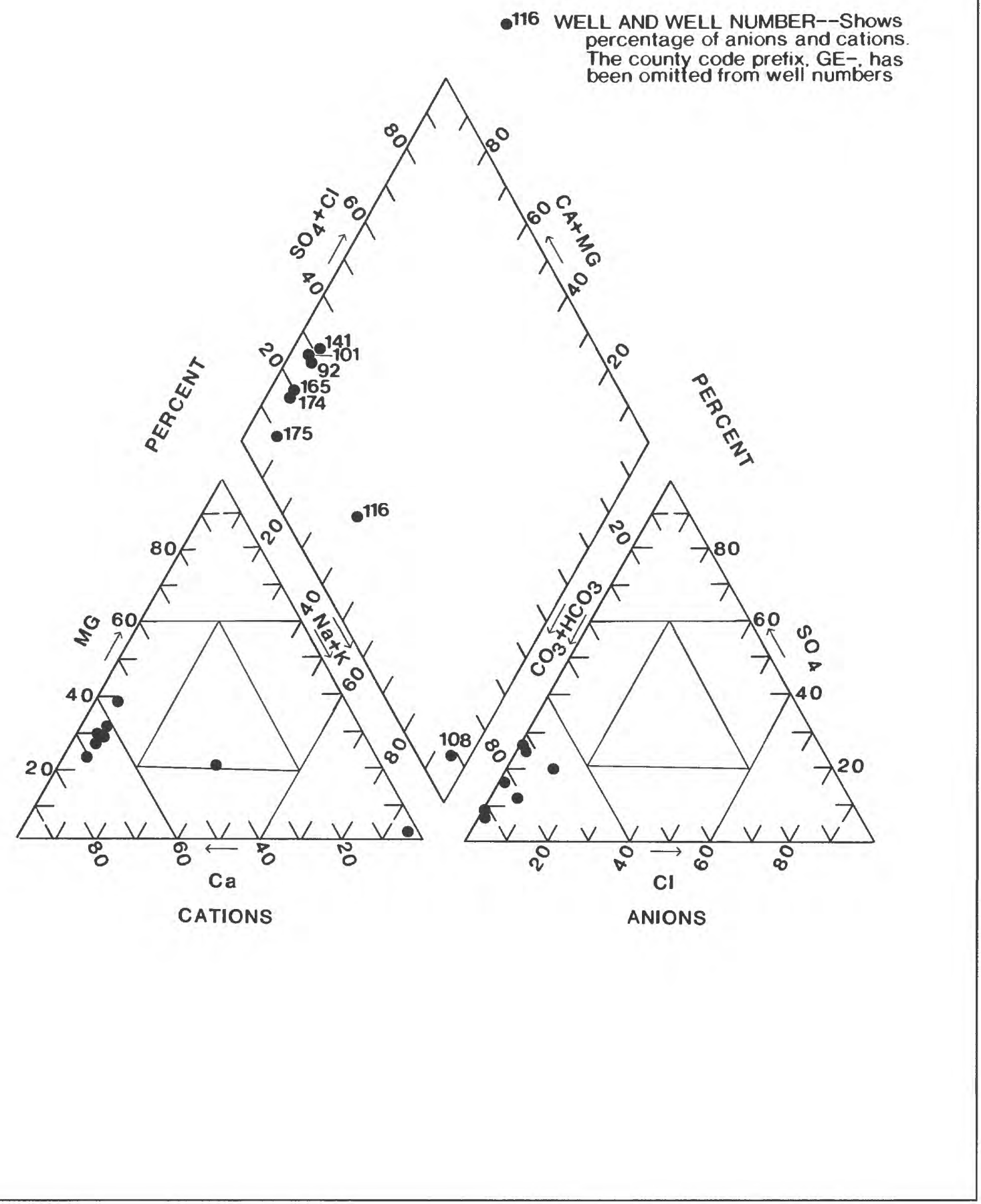

Figure 14.--Piper diagram showing variation in the chemical character of waters from the glacial deposits in Geauga County. Ohio. 


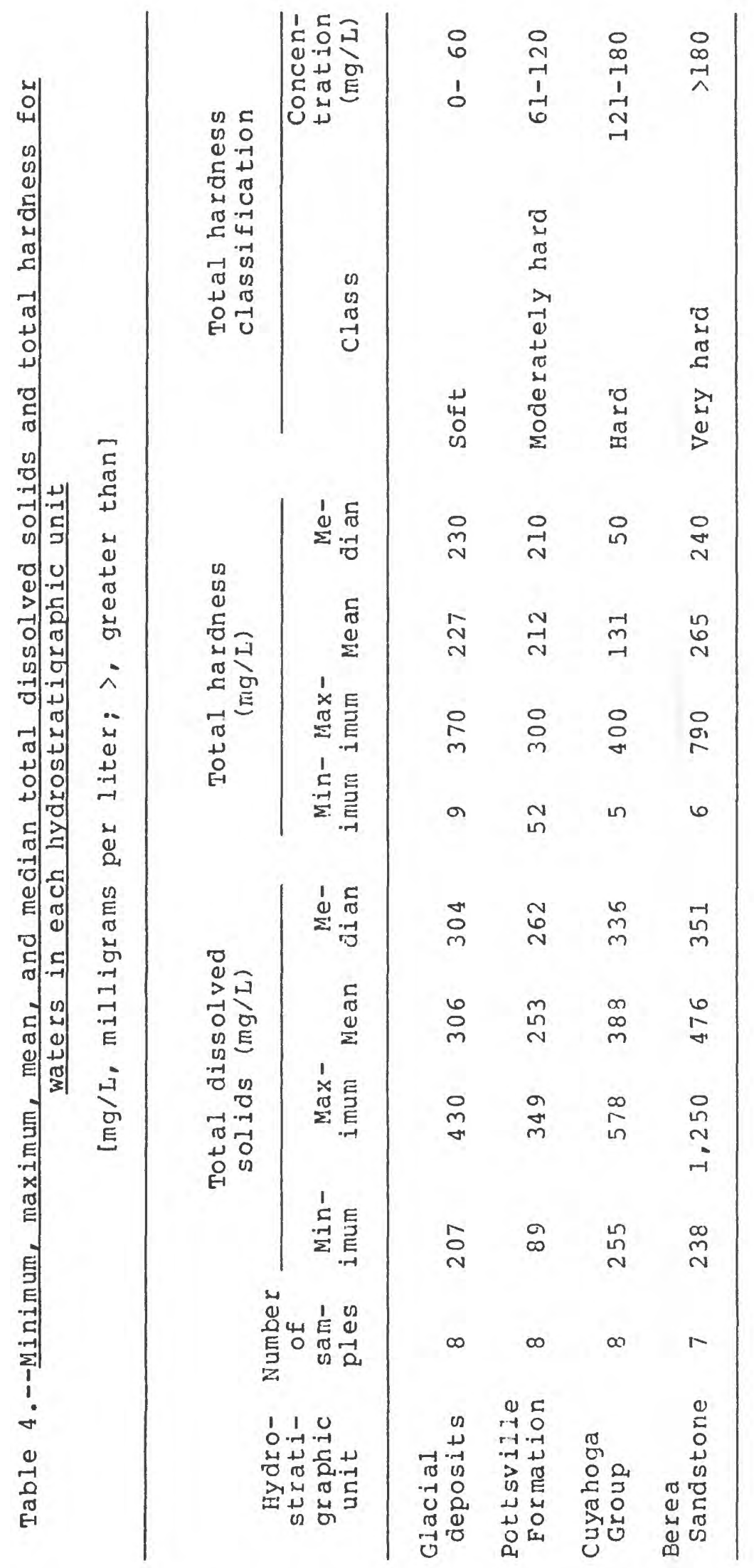




\section{EXPLANATION}

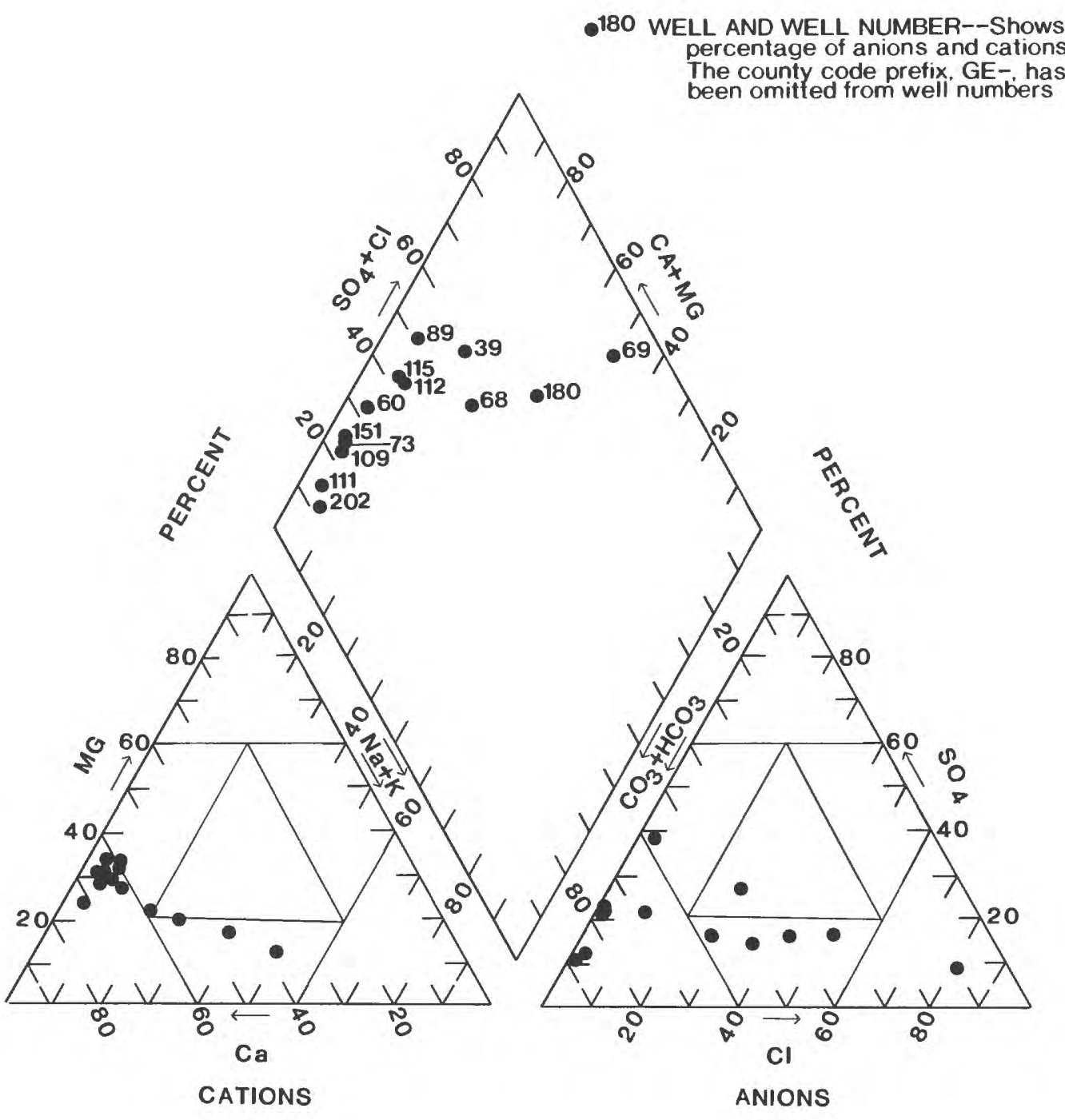

Figure 15.--Piper diagram showing variation in the chemical character of waters from the Pottsville Formation in Geauga County. Ohio. 
The TDS concentration of the eight waters unaffected by road salt is low (tables 3 and 4), indicating that none of these waters is highly mineralized. The total hardness of these wells is highly variable. The summary statistics listed on table 4 indicate that waters in the Pottsville Formation can range in total hardness from soft to very hard.

None of these waters exceeds USEPA MCLs or SMCLs for nitrate, sulfate, fluoride, barium, or methylene-blue active substances. The sample collected from well GE-69 exceeds the USEPA SMCLs for chloride and TDS. Samples from wells GE-73, GE-109, GE-115, GE-151, and GE-202 exceed the USEPA SMCL for iron. Samples from wells GE-109, GE-115, GE-151, and GE-202 exceed the USEPA SMCL for manganese. The SMCLs for chloride, TDS, iron, and manganese are nonhealth-based.

\section{Cuyahoga Group}

Water samples were collected and analyzed for eight wells completed in the Cuyahoga Group (fig. 5, table 3). Most of these waters (GE-55, GE-67, GE-105, GE-169, and GE186) are of the sodium bicarbonate-type (fig. 16). Although the anion character of the remaining waters is either bicarbonate or bicarbonate/sulfate in character, their relative concentrations of calcium and magnesium are nearly the same. The waters from wells GE-48 and GE-77 are mixed cation bicarbonate waters, whereas the water from well GE147 is a mixed cation bicarbonate/ sulfate water.

The TDS concentrations of waters from the Cuyahoga Group is slightly higher than those in the glacial deposits and the Pottsville Formation (tables 3 and 4). The total hardness in the Cuyahoga Group, however, generally is lower than those in the glacial deposits and the Pottsville Formation, indicating that waters in the Cuyahoga Group are slightly softer than in the other hydrostratigraphic units.

None of these waters exceeds USEPA MCLs or SMCLs for nitrate, chloride, sulfate, fluoride, barium, manganese, or methylene-blue active substances. Samples from wells GE-48 and GE-77 exceed the USEPA SMCL for iron, whereas samples from wells GE77, GE-105, and GE-147 exceed the USEPA SMCL for TDS.

\section{Berea Sandstone}

Water samples were collected and analyzed for seven wells completed in the Berea Sandstone (fig. 5, table 3). Two of these waters (GE-102 and GE-126) are calcium bicarbonate-types, whereas two others (GE-120 and GE-159) are sodium bicarbonatetypes (fig. 17). Two of the other three waters (GE-103 and GE-104) are a bicarbonate type but range from calcium, magnesium, to mixed cation-types, respectively. Water from well GE-122 is a calcium-magnesium type but is markedly different in anion type because of predominant sulfate. 


\section{EXPLANATION}

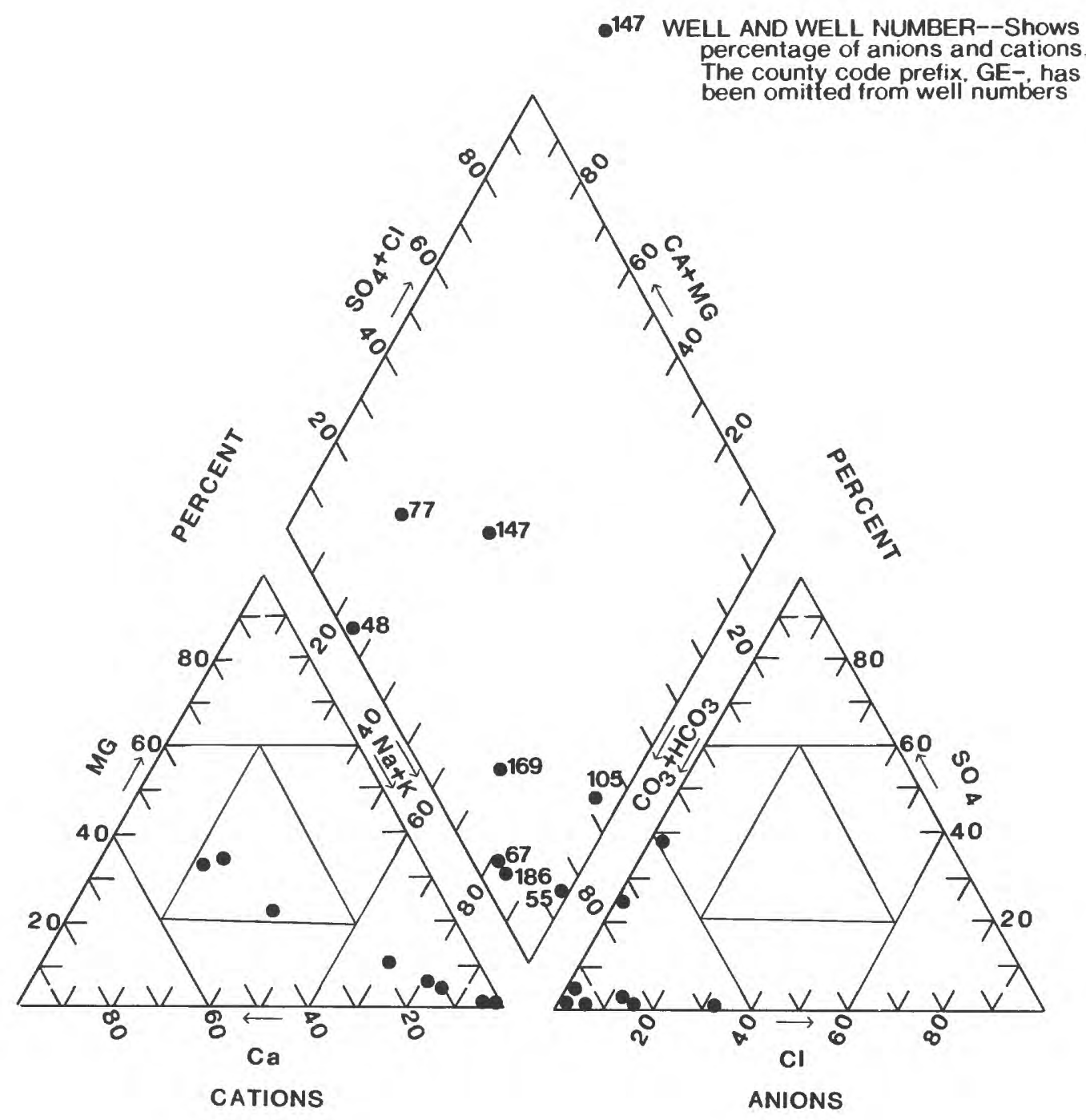

Figure 16.--Piper diagram showing variation in the chemical character of waters from the Cuyahoga Group in Geauga County, Ohio. 


\section{EXPLANATION}

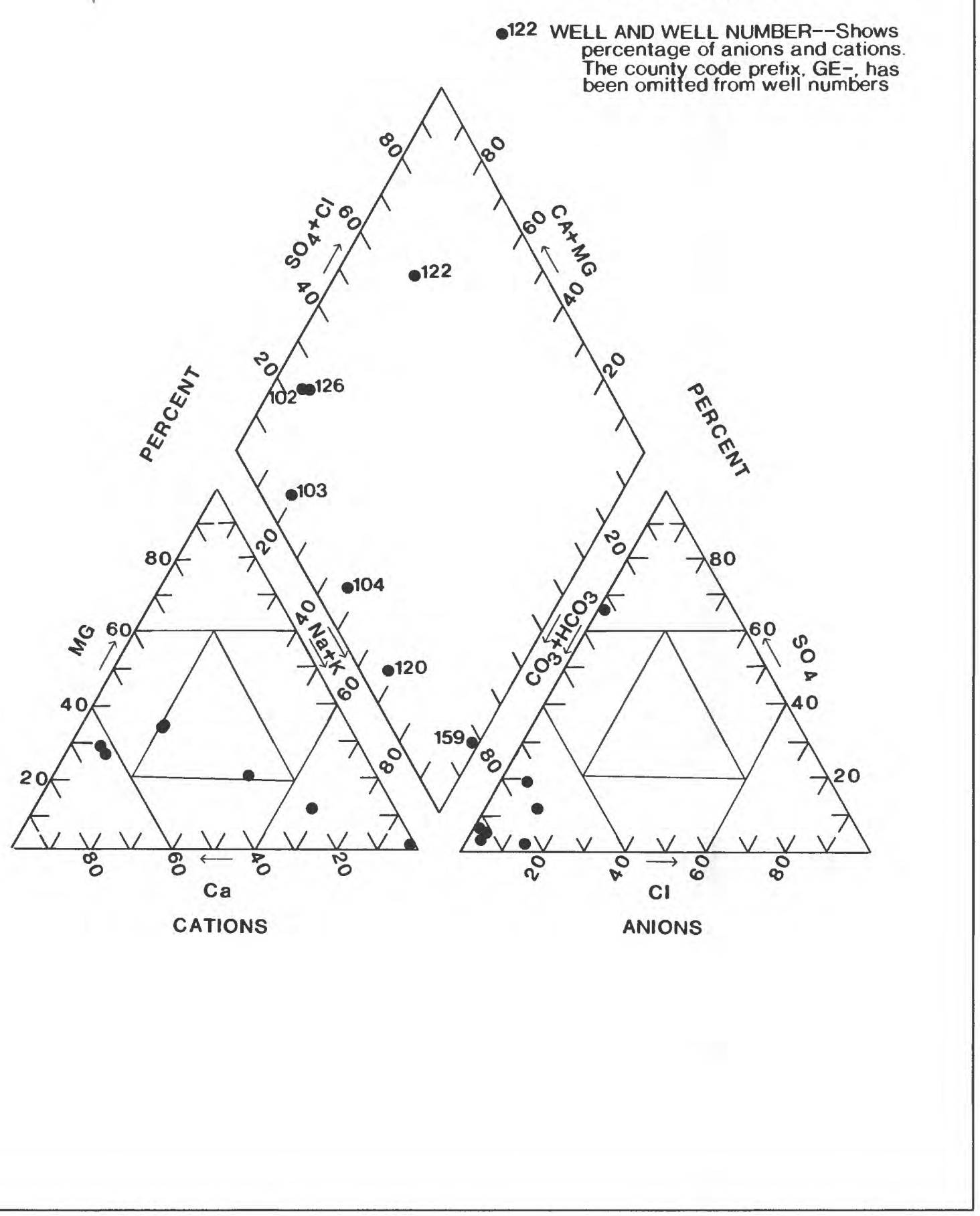

Figure 17.--Piper diagram showing variation in the chemical character of waters from the Berea Sandstone, Geauga County, Ohio. 
The TDS concentration of the waters from the Berea Sandstone is highly variable, and some waters are moderately mineralized (tables 3 and 4). The total hardness of these waters also is highly variable (table 3 ), indicating that the waters from the Berea Sandstone range in hardness from soft to very hard (table 4).

None of these waters exceeds USEPA MCLs or SMCLs for concentrations of nitrate, chloride, fluoride, barium, or methylene-blue active substances. The sample from well GE-122 exceeds the USEPA SMCL for sulfate. Samples from wells GE-103, GE-104, GE-122, and GE-126 exceed the USEPA SMCL for iron. Samples from wells GE-103, GE-122, and GE-126 exceed the USEPA SMCL for manganese. The sample from well GE-122 exceeds the USEPA SMCL for TDS.

\section{Regional Variations}

As ground water moves within a flow system, systematic changes in its composition commonly occur. In general, as water flows from recharge areas to discharge areas, the TDS content of the water and the concentrations of the major anions and cations increase. Shallow ground waters in recharge areas typically have lower TDS concentrations than waters deeper in the same flow system or shallow waters in discharge areas. The solution of carbon dioxide gas in the atmosphere in rainwater and the reaction of the weak acid thus formed with carbonate minerals in the soil zone results in recharge waters that are usually of calcium bicarbonate character. This is the case for many of the shallow ground waters in the surficial aquifers (glacial deposits and Pottsville Formation) in Geauga County. However, deeper ground waters in the Cuyahoga Group commonly are sodium bicarbonate waters.

Comparison of figure 16 with figures 14 and 15 also shows that waters in the surficial aquifers (glacial deposits and the Pottsville Formation) commonly are calcium bicarbonate waters, whereas waters from the Cuyahoga Group commonly are sodium bicarbonate waters. The change in the dominant cation from calcium to sodium within a geologic deposit containing an abundance of clay minerals suggests control by cation exchange. Cation exchange is the process whereby dissolved cations in ground water are exchanged for adsorbed cations on electrically charged surfaces of clay minerals. In fresh (dilute) waters, the dominant exchangeable cation is calcium (Drever, 1982). Marine clays typically have more adsorbed sodium than calcium. Accompanying the exchange of calcium for sodium on clays is the dissolution of any calcium carbonate minerals due to the decrease in calcium concentration in the ground water. As a result of the sodium for calcium ion-exchange process and concomitant carbonate equilibria in the marine shales of the Cuyahoga Group, the TDS concentrations of the waters within the Cuyahoga Group increases, and the total hardness decreases relative to the concentrations of these constituents in the shallower ground waters in the glacial deposits and the Pottsville 
Formation (table 4). The extent of the cation exchange effect on the ground waters is reflected in the straight-line trend from nearly calcium, magnesium character to the far corner of the sodium triangle in figure 16.

The natural softening of ground waters from cation exchange on the surface or in the crystalline structure of clay minerals commonly is observed in similar hydrogeologic conditions (Hem, 1985).

The change in dominant cations also can be seen by comparing the calcium:sodium (Ca:Na) ion ratio, in terms of milliequivalents per liter of waters from the glacial deposits and the Pottsville Formation with waters from the Cuyahoga Group. The table below summarizes these statistics.

\begin{tabular}{|c|c|c|c|c|}
\hline \multirow[b]{2}{*}{$\begin{array}{l}\text { Hydrostrati- } \\
\text { graphic unit }\end{array}$} & \multirow[b]{2}{*}{$\begin{array}{c}\text { Number of } \\
\text { samples }\end{array}$} & \multicolumn{3}{|c|}{$\mathrm{Ca}: \mathrm{Na}$ ratio } \\
\hline & & Mean & Median & $\begin{array}{l}\text { Standard } \\
\text { deviation }\end{array}$ \\
\hline Glacial deposits- - - - - & 8 & 9.60 & 11.3 & 5.90 \\
\hline Pottsville Formation- - - - & 8 & 13.8 & 14.9 & 5.97 \\
\hline Cuyahoga Group- - - - - & 8 & 0.664 & 0.150 & 0.830 \\
\hline Berea Sandstone- - - - - - & 7 & 3.12 & 2.40 & 3.48 \\
\hline
\end{tabular}

Figures $18-20$ show spatial variations in water quality, as represented by Stiff diagrams plotted on potentiometric-surface maps, in the glacial deposits and the Pottsville Formation (fig. 18) and the Berea Sandstone (fig. 20), and on a geographic base map for the Cuyahoga Group (fig. 19). Regional variations in the chemical character of the waters in these geologic units is indicated by changes in the shape of the Stiff diagrams. The relative positions of the wells within the local ground-water-flow system can be inferred from the equipotential contours. Local ground-water discharge areas generally are represented by the lowest equipotential lines, which commonly are shown along the bedrock outcrops and in pairs on either side of a buried valley. Local ground-water recharge areas generally are represented by the highest equipotential lines, which commonly are shown as closed contours underlying topographically high areas. 
The chemical character of shallow ground waters in the surficial glacial deposits and the Pottsville Formation does not vary significantly on a regional basis (fig. 18). There are no discernible trends in the chemical character of the waters related to flow patterns in these surficial aquifers. However, the localized contamination of well GE-69 by road salt stored at the State highway garage can be identified on this figure by the anomalous Stiff diagram, which indicates a sodium/calcium-chloride water.

Spatial variations in the chemical character of the waters in the Cuyahoga Group (fig. 19) appear to be related to the length of the open interval of the well, the hydraulic conductivity of the open interval, and the stratigraphic position of the open interval relative to the presence of overlying permeable glacial deposits. Data from drillers' logs indicate that the sodium bicarbonate waters commonly are obtained from wells (GE-55, GE-67, GE-105, GE-169, and GE-186) that are open to several tens of feet of shale overlain by 30 to $40 \mathrm{ft}$ of clay. These waters probably reflect the chemical character of most of the waters in the shales in the Cuyahoga Group; that is, those that have been naturally softened by the exchange process.

Waters of mixed-cation character in the Cuyahoga Group commonly are obtained from wells (GE-48, GE-77, and GE-147) that are completed in shale but have a relatively short open interval ( 5 to $10 \mathrm{ft}$ ) and are overlain by glacially derived sand and gravel deposits. Part of the water from these wells appears to be calcium bicarbonatetype water leaking downward from overlying glacial deposits. This calcium bicarbonate water has not passed through much of the shale and thus has not been appreciably softened. As a result, their chemical character is a mixture of the two water types. Presence of such waters does not appear to be related to the position of the well within the local flow system.

Some of the well waters in the Berea Sandstone reflect vertical leakage of sodium bicarbonate-type waters from the overlying Cuyahoga Group (fig. 20). Wells GE-120 and GE-159 are completed in sandstone overlain by $30 \mathrm{ft}$ or more of shale, and are located between local recharge and discharge areas where regional flow gradients are downward from the Cuyahoga Group into the Berea Sandstone. Calcium bicarbonatetype waters are from wells GE-102 and GE-126, which are located in ground-water discharge areas adjacent to buried valleys which contain calcium bicarbonate-type waters.

The waters of mixed-cation character in the Berea Sandstone (GE-103 and GE-104) are overlain by glacially derived sand and gravel or interbedded sand and clay deposits. The mixed-cation character of these waters probably results from vertical leakage from the overlying materials. GE-122 is a mixed cation-type water having an unknown origin. 


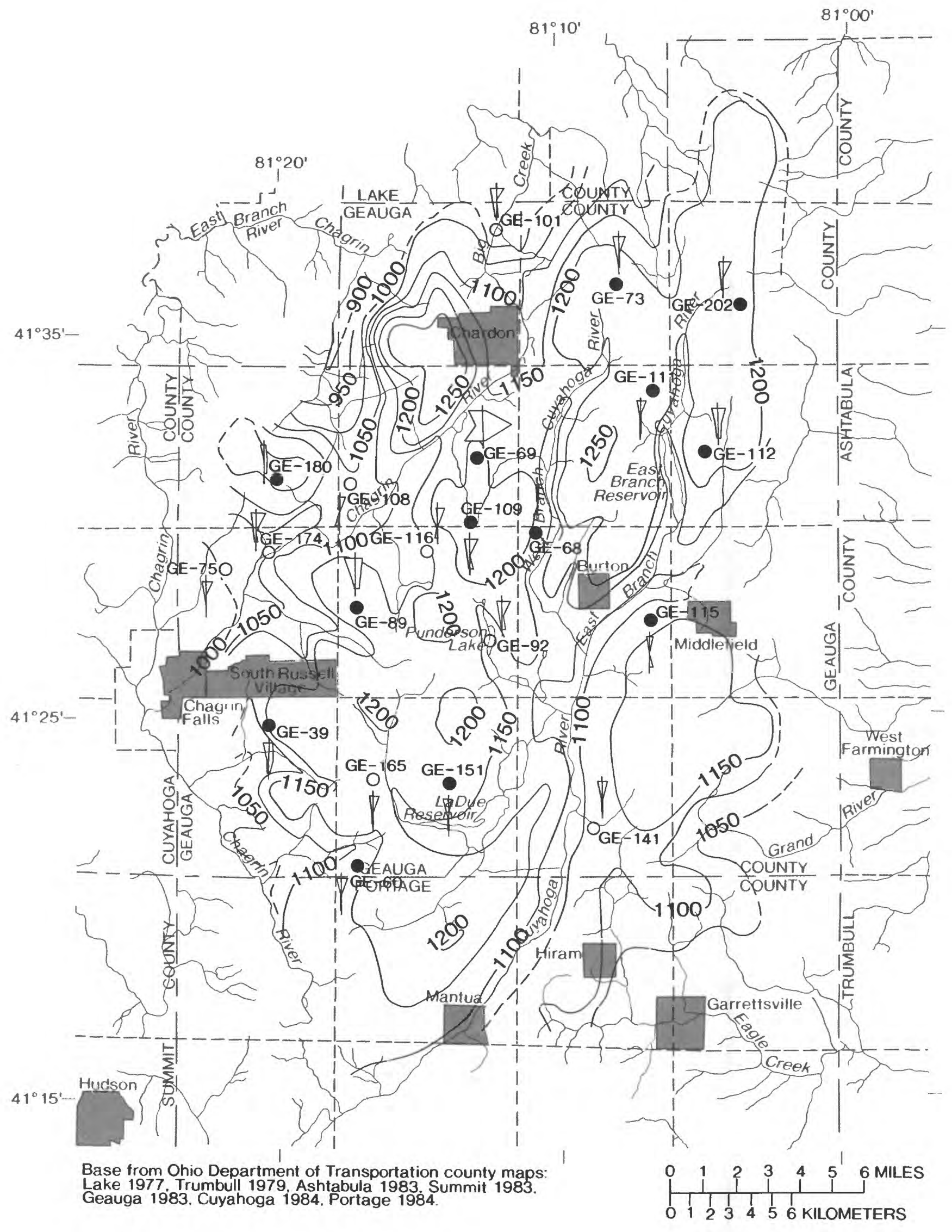




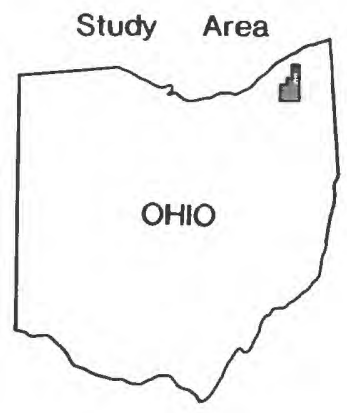

\title{
EXPLANATION
}

\author{
DIAGRAM SHOWING CONCENTRATIONS OF CATIONS \\ AND ANIONS \\ Calcium ( $\mathrm{Ca})$ Bicarbonate ( $\mathrm{HCO} 3)$ \\ Magnesium (Mg) Chhloride (CI) \\ Sodium ( $\mathrm{Na}$ ) ${ }_{\text {Sulfate ( }} \mathrm{SO}_{4}$ ) \\ and potassium $(\mathrm{K})$ \\ Cations 10 : 10 1 10 Anions \\ Millequlvalents per liter
}

\section{-GE-175 LOCATION OF SAMPLED WELL AND NUMBER IN THE POTTSVILLE FORMATION \\ OGE-73 LOCATION OF SAMPLED WELL AND NUMBER IN THE GLACIAL DEPOSITS \\ -1150- - WATER-TABLE CONTOUR--Shows altitude of water table. Dashed where approximately located. Contour interval $\mathbf{5 0}$ feet. Datum is sea level.}

Figure 18.--Stiff diagrams showing spatial variations in water quality relative to the potentiometric surface in the glacial deposits and the Pottsville Formation. 


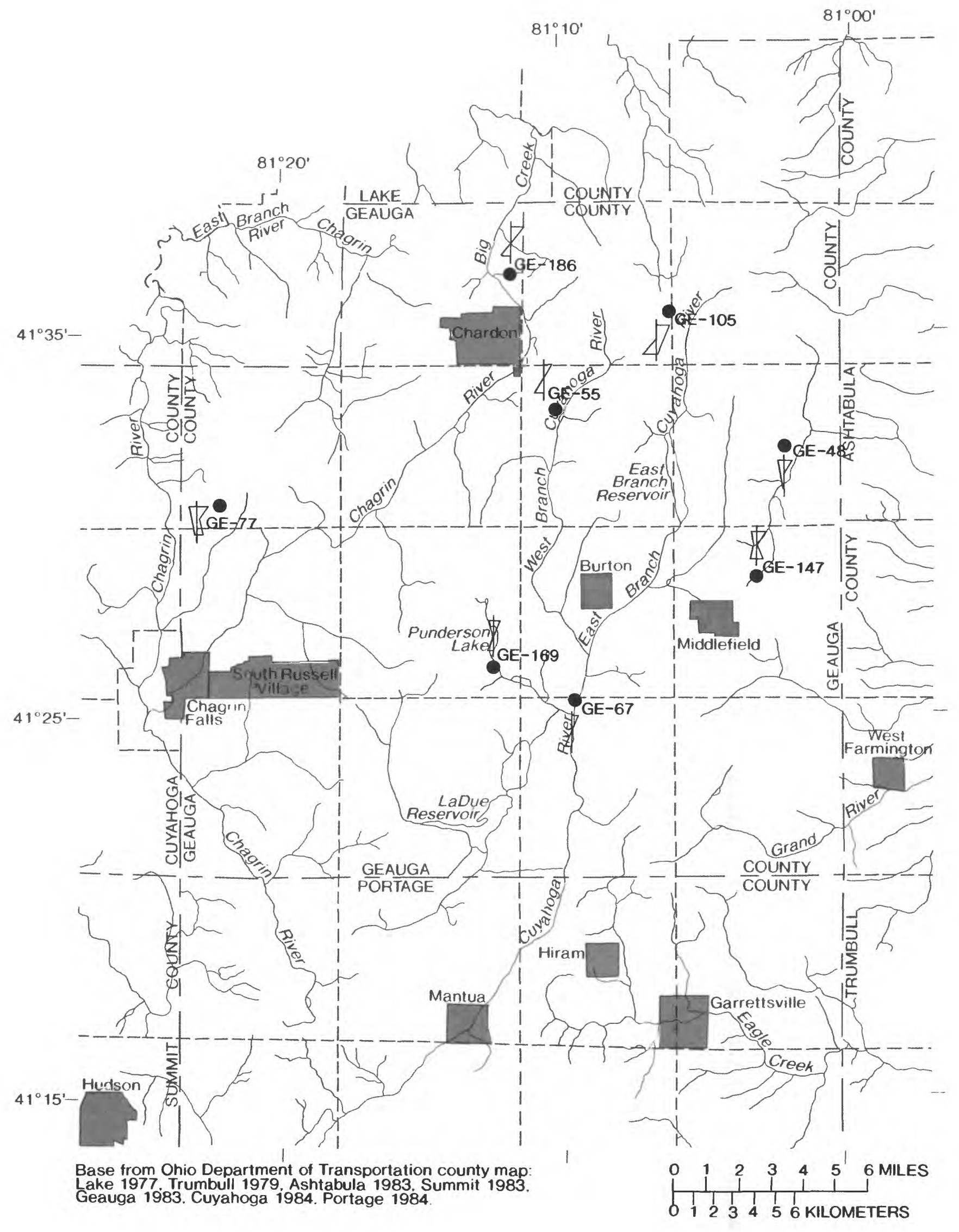




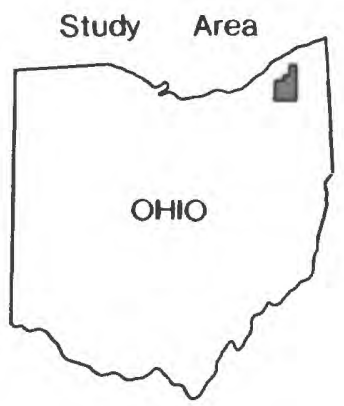

\section{EXPLANATION}

\section{DIAGRAM SHOWING CONCENTRATIONS OF CATIONS}

AND ANIONS

Calcium (Ca) Bicarbonate ( $\left.\mathrm{HCO}_{3}\right)$

Magnesium (Mg) Chloride (Cl)

Sodium ( $\mathrm{Na})$ Sulfate ( $\mathrm{SO}_{4}$ ) and potassium (K)

$$
\begin{aligned}
& \text { Cations } 10 \text { l } \\
& \text { Millequlva!ents per liter }
\end{aligned}
$$

GE-169 LOCATION OF SAMPLED WELL AND NUMBER 


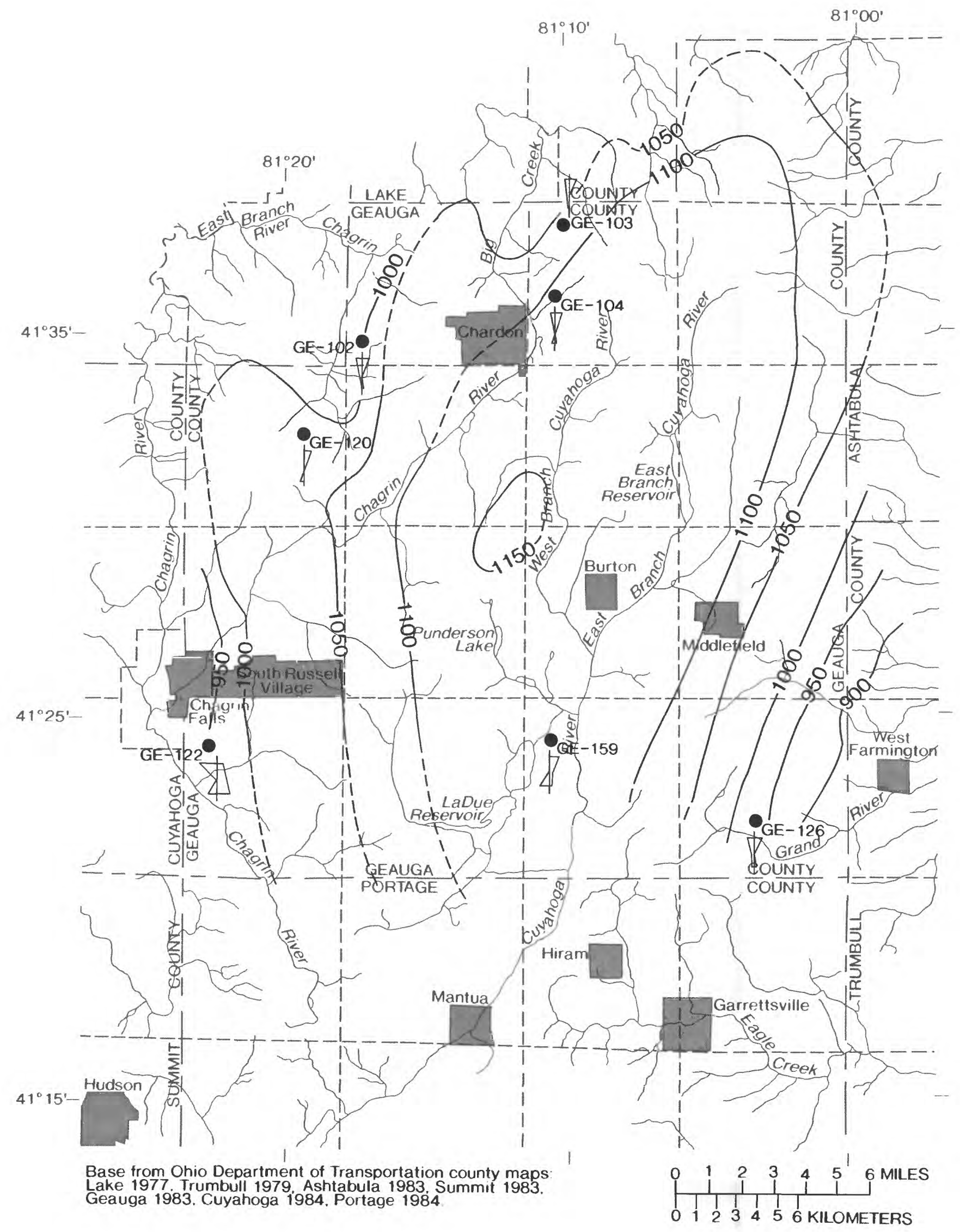




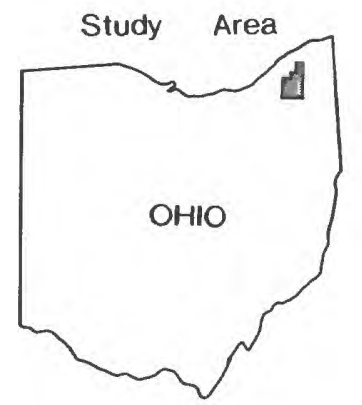

EXPLANATION

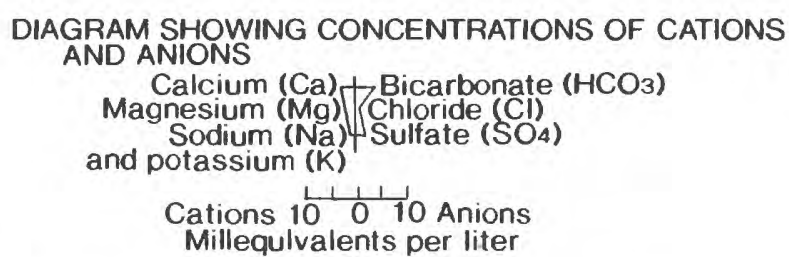

GE-122 LOCATION OF SAMPLED WELL AND NUMBER

-1150-- WATER-LEVEL CONTOUR--Line of equal altitude of water surface Shows altitude of water levei Dashed where approximately located. Contour interval 50 feet

Datum is sea level

Figure 20--Stiff diagrams showing spatial variatıons in water quality relative to the potentiometric surface in the Berea Sandstone 


\section{Temporal Variations in Quality}

Water-quality data for wells sampled as part of the 1978 and 1980 projects (Nichols, 1980 ) were compared with the data from the current (1985 to 1988) project to determine whether temporal variations in regional ground-water quality had occurred during the period between studies. The water quality of 12 wells was determined two or more times during these three projects. Three of these wells (GE-92, GE-108, and GE-116) are completed in the glacial deposits, four (GE-39, GE-68, GE-73, and GE-112) in the Pottsville Formation, four (GE-48, GE-55, GE-67, and GE-105) in the Cuyahoga Group, and one (GE-103) in the Berea Sandstone. The results of these analyses are displayed as a series of plots of concentration against time (figs. 21-24).

Specific conductance and TDS commonly are used as indicators to determine if the concentration of major dissolved constituents has changed with time. Minor fluctuations in specific conductance and TDS are common. The magnitude of these fluctuations depends on variations in recharge (unconfined aquifers), leakage (confined aquifers), induced stream infiltration, and sampling and analytical error. Shallow, unconfined aquifers commonly manifest greater fluctuations in specific conductance and TDS than deeper, confined aquifers because of shorter flow paths to local discharge areas and shorter residence times.

Figure 21 shows temporal changes in specific conductance and TDS, respectively. The variations from most of the wells shown on these figures are minor. However, the specific conductance of well GE-39, which is completed in the Pottsville Formation, varied by $200 \mathrm{uS} / \mathrm{cm}$ between 1978 and 1980 . On the basis of the slope of the regression line shown on figure 13, this is equivalent to about $130 \mathrm{mg} / \mathrm{L}$ TDS. Although the measured variations in either parameter are not unexpected for a well completed in a surficial, unconfined aquifer, they may indicate an influence of deicing salts.

Figure 22 shows temporal variations in the concentrations of the major cations, calcium, magnesium, and sodium. The greatest temporal variations in calcium concentration are at wells GE-108 and GE-116, which are completed in glacial deposits, and at wells GE-39 and GE-112, which are completed in the Pottsville Formation. The magnitude of these variations is less than $30 \mathrm{mg} / \mathrm{L}$. The greatest temporal variations in magnesium concentration are at wells GE-108 and GE-116, which are completed in glacial deposits, and at well GE-39, which is completed in the Pottsville Formation. The magnitude of these variations is about $10 \mathrm{mg} / \mathrm{L}$. Samples from wells GE-108 and GE-116 also display the greatest temporal variations, about $50 \mathrm{mg} / \mathrm{L}$, in sodium concentration.

These temporal variations are typical of cation variations observed in water from wells near local discharge areas in shallow, unconfined aquifers. Because most of these wells are located in local ground-water discharge areas, none of the measured variations 


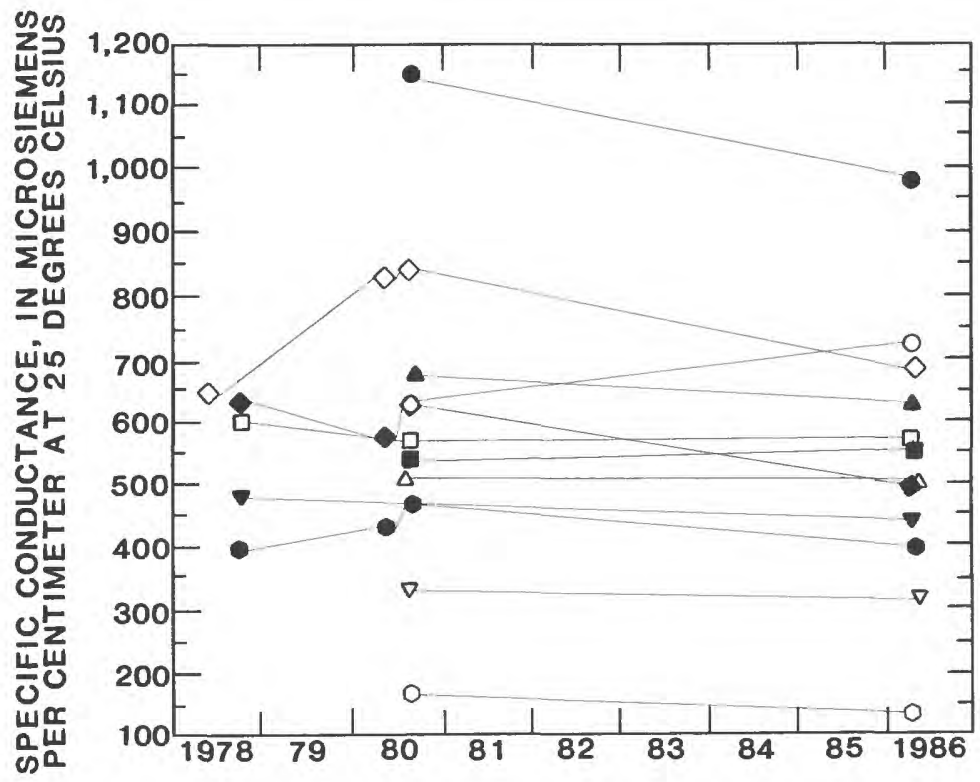

EXPLANATION
$\triangle \mathrm{GE}-116$
$\nabla$ GE -108
$\diamond$ GE-39
- GE-68
- GE-112
- GE-92
- GE-48
- GE-55
- GE-67
- GE-105
口 GE-73
- GE-103

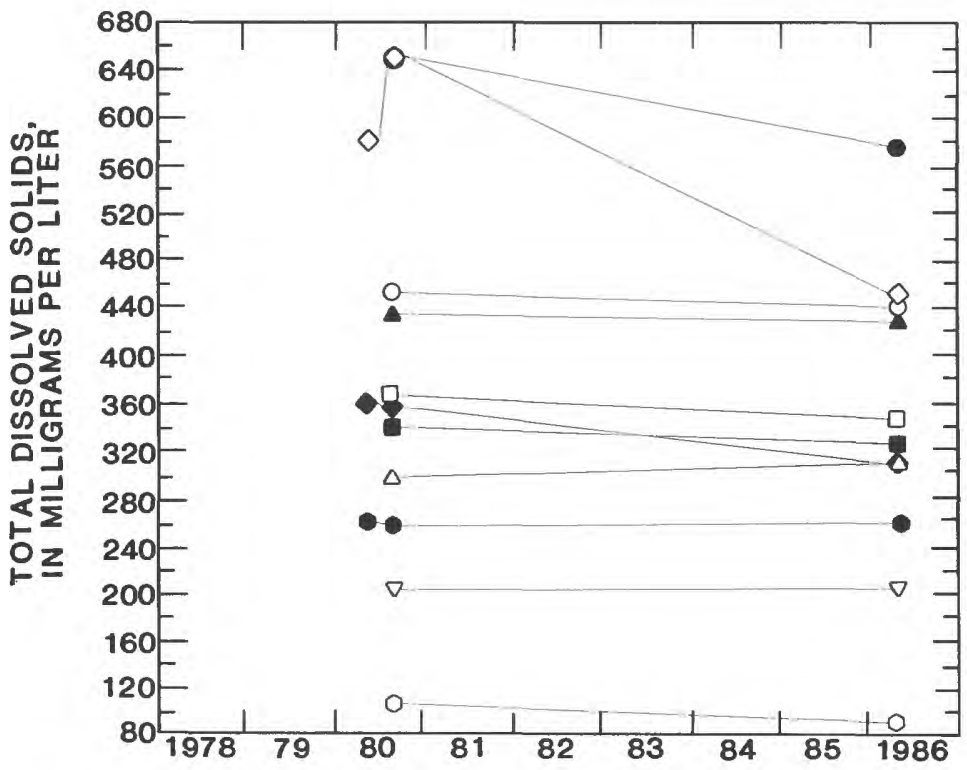

\section{EXPLANATION}

$\triangle \mathrm{GE}-116$

$\nabla$ GE-108

口 GE-73

$\diamond$ GE-39

- GE-68

- GE-112

- GE-92

- GE-55

- GE-67

- GE-105

- GE-103

Figure 21.--Variability of specific conductance and total dissolved solids concentration in ground water from selected wells in Geauga County, Ohio, from May 1978 through May 1986 (lines connecting the symbols are for visual reference only and do not imply continuity of data). 


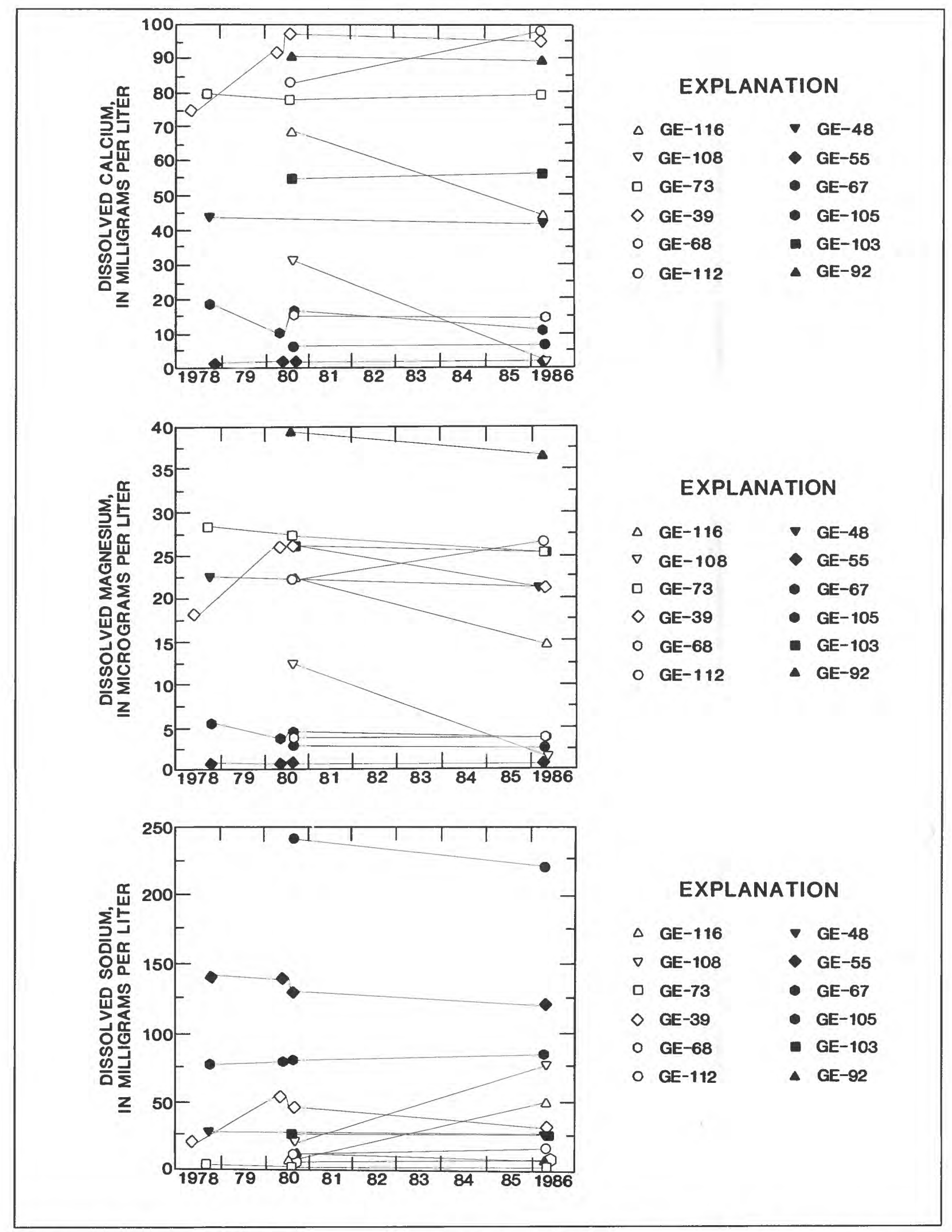

Figure 22.--Variability of concentrations of dissolved calcium, dissolved magnesium, and dissolved sodium in ground water from selected wells in Geauga County.

Ohio. from May 1978 through May 1986 (lines connecting the symbols are

for visual reference only and do not imply continuity of data). 


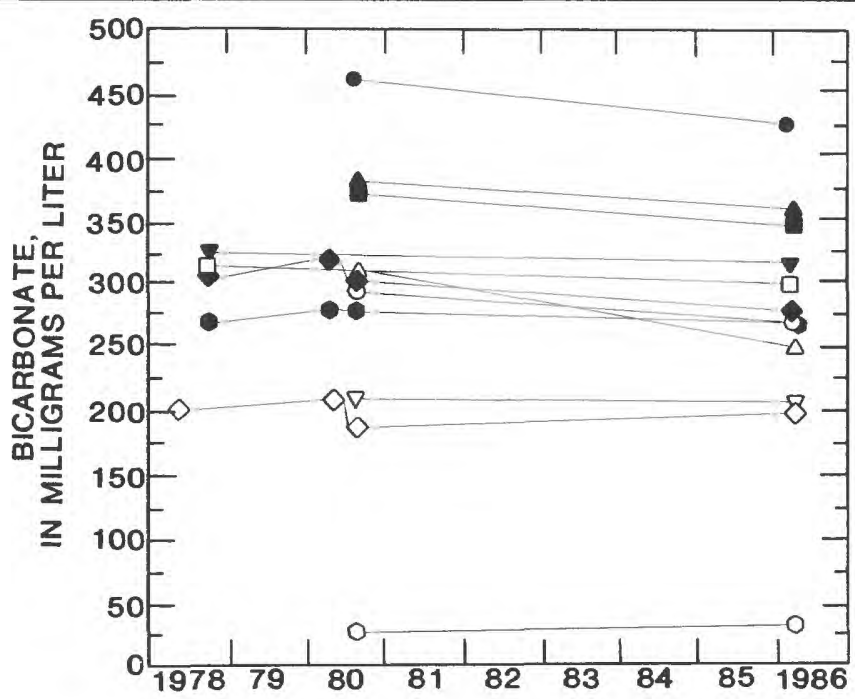

\section{EXPLANATION}
$\triangle$ GE-116
- GE-48
$\nabla$ GE-108
- GE-55
ㅁ GE-73
- GE-67
$\diamond$ GE-39
- GE-105
- GE-68
- GE-103
○ GE-112
- GE-92

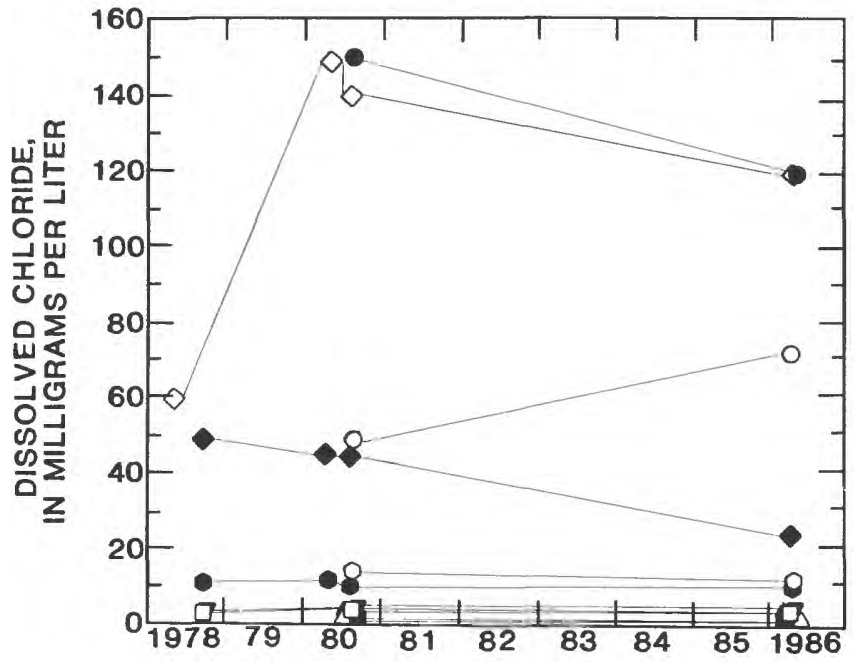

\section{EXPLANATION}
$\triangle$ GE-116
- GE-48
$\nabla$ GE-108
- GE-55
ㅁ GE-73
- GE-67
$\diamond$ GE-39
- GE-105
- GE-68
- GE-103
○ GE-112
- GE-92

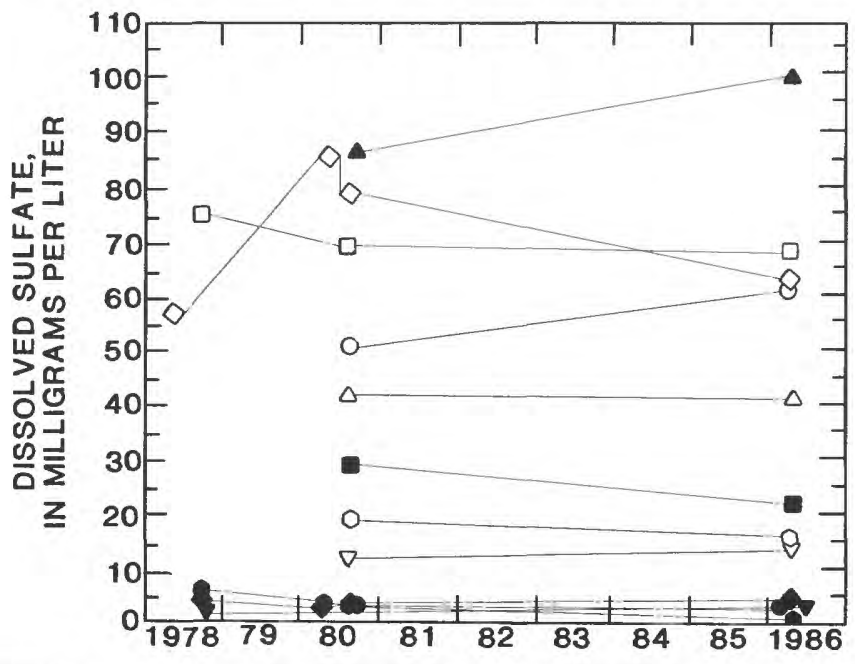

\section{EXPLANATION}
$\triangle$ GE-116
- GE-48
$\nabla$ GE-108
- GE-55
$\mathrm{GE}-73$
- GE-67
$\diamond$ GE-39
- GE-105
- GE-68
- GE-103
- GE-112
- GE-92

Figure 23.--Variability of concentrations of bicarbonate, dissolved chloride, and dissolved sulfate in ground water from selected wells in Geauga County. Ohio, from May 1978 through May 1986 (lines connecting the symbols are for visual reference only and do not imply continuity of data). 


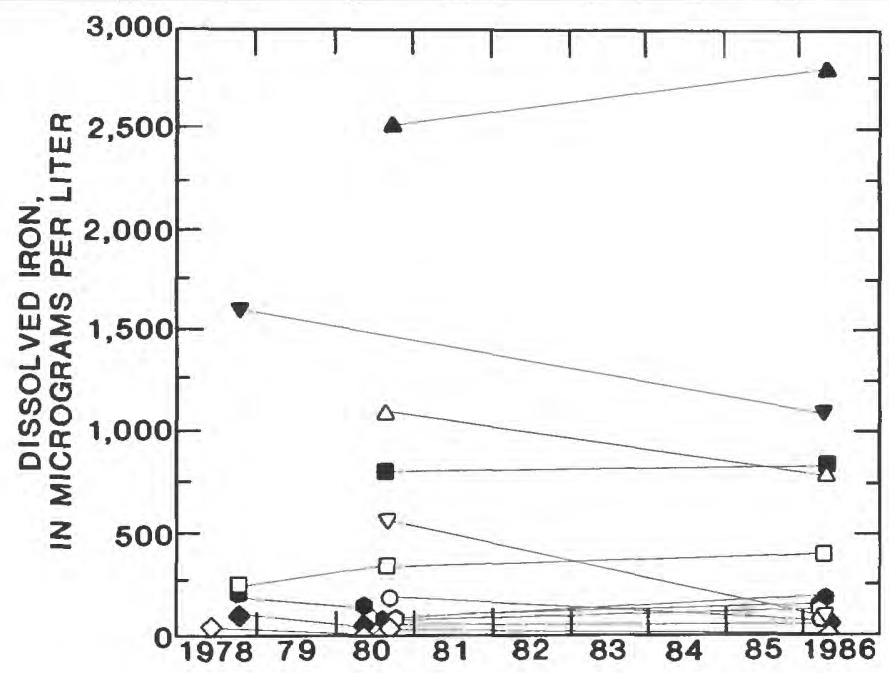

\section{EXPLANATION}
$\triangle$ GE- 116
- $\mathrm{GE}-48$
$\nabla$ GE-108
- GE-55
$\mathrm{GE}-73$
- GE-67
$\diamond$ GE-39
- GE-105
- GE-68
- GE-103
- GE-112
- GE-92

\section{EXPLANATION}
$\triangle \mathrm{GE}-116$
- GE-48
$\nabla$ GE- 108
- GE-55
$\square$ GE-73
- GE-67
$\diamond$ GE-39
- GE-105
- GE-68
- GE-103
O GE-112
- GE-92

\section{EXPLANATION}
$\triangle$ GE- 116
- GE-92
口 GE-73
- GE-55
$\diamond$ GE-39
- GE-67
- $\mathrm{GE}-68$
- GE-105
- GE-112

Figure 24.--Variability of concentrations of dissolved iron, total organic carbon, and dissolved ammonia in ground water from selected wells in Geauga County, Ohio, from May 1978 through May 1986 (lines connecting the symbols are for visual reference only and do not imply continuity data). 
in major cation concentrations is unexpected. None of the waters from wells completed in the Cuyahoga Group (GE-48, GE-55, GE-67, and GE-105) or the Berea Sandstone (GE-103) display any notable temporal variations in major cation concentrations. The lack of temporal variation in major cation concentrations is not unexpected and is typical for wells completed in deeper geologic units in more isolated and confined parts of a flow system. Most of these wells are adjacent to ground-water-discharge areas in the buried valleys.

Figure 23 shows temporal variations in the concentrations of bicarbonate, chloride, and sulfate. None of the data indicate any remarkable variation in bicarbonate concentration. Data from well GE-39, however, display nearly a $100-\mathrm{mg} / \mathrm{L}$ increase in chloride concentration between 1978 and 1980. The well is completed in a surficial, unconfined part of the Pottsville Formation and is at the intersection of State Route 306 and U.S. Route 422. The elevated chloride concentration measured in late 1979 and early 1980 may be related to the use of a deicing agent. Increases in sodium and calcium concentrations were concomitant with the increase in chloride concentration. Calcium chloride and sodium chloride routinely are applied as deicing salts on roads in northeastern Ohio. The USEPA SMCL for chloride in drinking waters is $250 \mathrm{mg} / \mathrm{L}$. SMCLs are used to regulate undesirable substances that are not normally toxic except at high concentrations, but that may restrict the usability of water or reduce its esthetic quality. The variations in chloride concentration for other wells are smaller, as are those for sulfate concentration (fig. 23).

Figure 24 shows temporal variations in the concentrations of iron, total organic carbon, and dissolved ammonia (as nitrogen). None of the well waters varied appreciably in dissolved iron or ammonia concentrations. In contrast, the concentration of total organic carbon (TOC) increased substantially in waters from well GE-105, but decreased in waters from well GE-112. Although most ground waters contain small amounts of organic carbon, concentrations above a few $\mathrm{mg} / \mathrm{L}$ may indicate contamination from synthetic organic compounds, landfill leachate, septic-system effluent, or oilfield brine.

Well GE-105 is completed in the shales of the Cuyahoga Group and, according to the driller's log, is $220 \mathrm{ft}$ deep, contains $84 \mathrm{ft}$ of casing, and produced a clear water after a 3-hour well-acceptance test. The shale is overlain by about $80 \mathrm{ft}$ of stratified sands and clays. The large increase in TOC concentration in this well, in conjunction with $\mathrm{Br}: \mathrm{Cl}$ data to be presented in the following section of this report, suggests that it may be affected by oilfield brine. The other variations in TOC concentrations are not remarkable and probably represent normal background variations, except those for well GE-112, which appears to be affected by highway deicing salts.

None of the historic analyses of samples collected in wells in Geauga County (Nichols, 1980) indicates that nitrate concentrations exceed the USEPA MCL of $10 \mathrm{mg} / \mathrm{L}$. 


\section{Effects of Oil and Gas Development and Highway Deicing on Quality}

Geauga County is located in a region of oil and gas production as well as one of uncommonly high snowfall; thus, many residents are concerned about the potential effects of brine-disposal practices and application of highway deicing salts on local ground-water quality. In November 1986, brine samples were collected from three oil and gas wells. Two of these wells (GECL-CNG\#634 and GECL-BAPTIST\#1) produce brine from the Clinton zone in Geauga County, whereas the other well (SUNE-STOW\#2) produces brine from the Newburg zone in Summit County. The Clinton zone is a drillers' name for one or more of the three sandstone units that are present in the Albion Sandstone of Silurian age. The Newburg zone is a drillers' name for a porous, water-producing, carbonate zone that is present within a thick sequence of carbonate rocks referred to by drillers in Ohio as the Big Lime. This unit extends stratigraphically from the base of the Lockport Dolomite (Middle Silurian) to the top of the Delaware or Onondaga Limestone (Middle Devonian). Table 5 lists the chemical constituents and physical properties of these brines.

The construction of mixing curves for uncontaminated ground waters, oilfield brine, and halite-solution brine (such as derived from diluted highway deicing salts) on a diagram of bromide:chloride $(\mathrm{Br}: \mathrm{Cl})$ ratios plotted against chloride concentrations (fig. 25) is a method commonly used to differentiate between the different sources of salinity (deicing salts and oilfield brine) in dilute (less than $10,000 \mathrm{mg} / \mathrm{L}$ TDS) ground waters (D.O. Whittemore, Kansas Geological Survey, 1984, 1988; oral commun., 1989). Although other methods, including sodium:chloride, calcium:chloride, magnesium:chloride, and sulfate:chloride ratios have been used in attempts to distinguish between different salinity sources, most of these ion ratios are subject to error because of ion-exchange processes, mineral precipitation, or oxidation-reduction reactions. Bromide and chloride generally are the two most conservative constituents in natural ground waters (Whittemore, 1988). Consequently, $\mathrm{Br}: \mathrm{Cl}$ ratios are not susceptible to the same sources of error as techniques based on other ion pairs.

$\mathrm{Br}: \mathrm{Cl}$ weight ratios were computed from the 1986 water-quality data and plotted on figure 25 against the range of measured chloride concentrations for local ground waters and brines, as listed on tables 3 and 5 . Bromide concentrations were determined by use of method I-2129-85, which accounts for common ion interference from iodide (Fishman and Friedman, 1985). Mixing curves of $\mathrm{Br}: \mathrm{Cl}$ ratios and chloride concentrations representing the boundaries of various mixtures of oilfield brines and halite-solution brines with local ground waters were calculated and plotted on the diagram. The zones delineated by these curves are used to identify mixtures of local ground waters represented by zone A, with the two end-point waters, oilfield brine and halite-solution brine, represented by points $B$ ' and $C^{\prime}$ ' (fig. 25), respectively. These curves were based on the following mixing equation (Whittemore, 1988): 
where

$$
\mathrm{C}_{\mathrm{MIX}}=\mathrm{C}_{1} \mathrm{~V}+\mathrm{C}_{2}(1-\mathrm{V})
$$

$\mathrm{C}_{\mathrm{MIX}}$ is the concentration of bromide (or chloride) in the mixture,

$\mathrm{C}_{1}$ is the concentration of bromide (or chloride) in the first end-point water,

$\mathrm{C}_{2}$ is the concentration of bromide (or chloride) in the second end-point water, and

$\mathrm{V}$ is the volume fraction of the first end-point water.

The upper and lower boundaries of zone $\mathrm{A}$ were computed for $\mathrm{Br}: \mathrm{Cl}$ ratios that encompass all of the measured $\mathrm{Br}: \mathrm{Cl}$ ratios for ground waters in Geauga County, starting at the lowest measured chloride concentration (end points $\mathrm{A}^{\prime}$ and $\mathrm{A}^{\prime}$ ' '). The upper and lower boundaries of zone $\mathrm{B}$, which represents mixtures of oilfield brine with local ground waters, were computed to bracket the measured $\mathrm{Br}: \mathrm{Cl}$ ratios (point $\mathrm{B}^{\prime}$ ) from the three local oilfield brines by \pm 15 percent. The upper and lower boundaries of zone $C$, which represents mixtures of halite-solution brine with local ground waters, were computed to bracket the measured $\mathrm{Br}: \mathrm{Cl}$ ratios from road salts used in northeastern Ohio (Knuth and others, 1990). The percentage range for the endpoints bracketing points B ' and C' on figure 25 were based on combined estimates of natural variation and analytical error (D.O. Whittemore, Kansas Geological Survey, oral commun., 1989). The chloride boundary for zone A was chosen as that chloride concentration above which zones B and $\mathrm{C}$ were separate. Zone $\mathrm{D}$ represents waters that are either a mixture of local ground water with oilfield brine and halite-solution brine, or other local natural-formation waters.

Waters from most of the wells sampled in Geauga County plot within zone A and primarily represent unmixed ground waters, although the few points with the lowest $\mathrm{Br}: \mathrm{Cl}$ in zone $\mathrm{A}$ close to zone $\mathrm{C}$ could possibly reflect small increases in chloride from road salt. The water from well GE-69 plots within the middle of zone $\mathrm{C}$, which indicates that it is a mixture of local ground water and halite-solution brine. This is to be expected because this well is located only a few hundred feet away from a storage area for road salt. The waters from wells GE-39, GE-89, GE-112, and GE-180 also plot within zone C. All of these wells are located along major highways (fig. 5) and, therefore, are very susceptible to ground-water contamination from the application of deicing salts. Well GE-180 is located near the Chester Township highway garage that maintained an uncovered salt-storage pile for many years. Several wells in the area of the salt-storage pile 
Table 5.--Listing of concentrations of chemical constituents and physical properties of brines from the Newburg zone in Sumit County and the CIinton zone in Geauga county and from selected residential wells in Geauqa County, ohio

$\left[{ }^{\circ} \mathrm{C}\right.$, degrees Celsius; mg/L, milligrams per liter; ug/L, micrograms per 1 iter; $\mu \mathrm{S} / \mathrm{cm}, \mathrm{micro-}$ siemens per centimeter at 25 degrees Celsius]

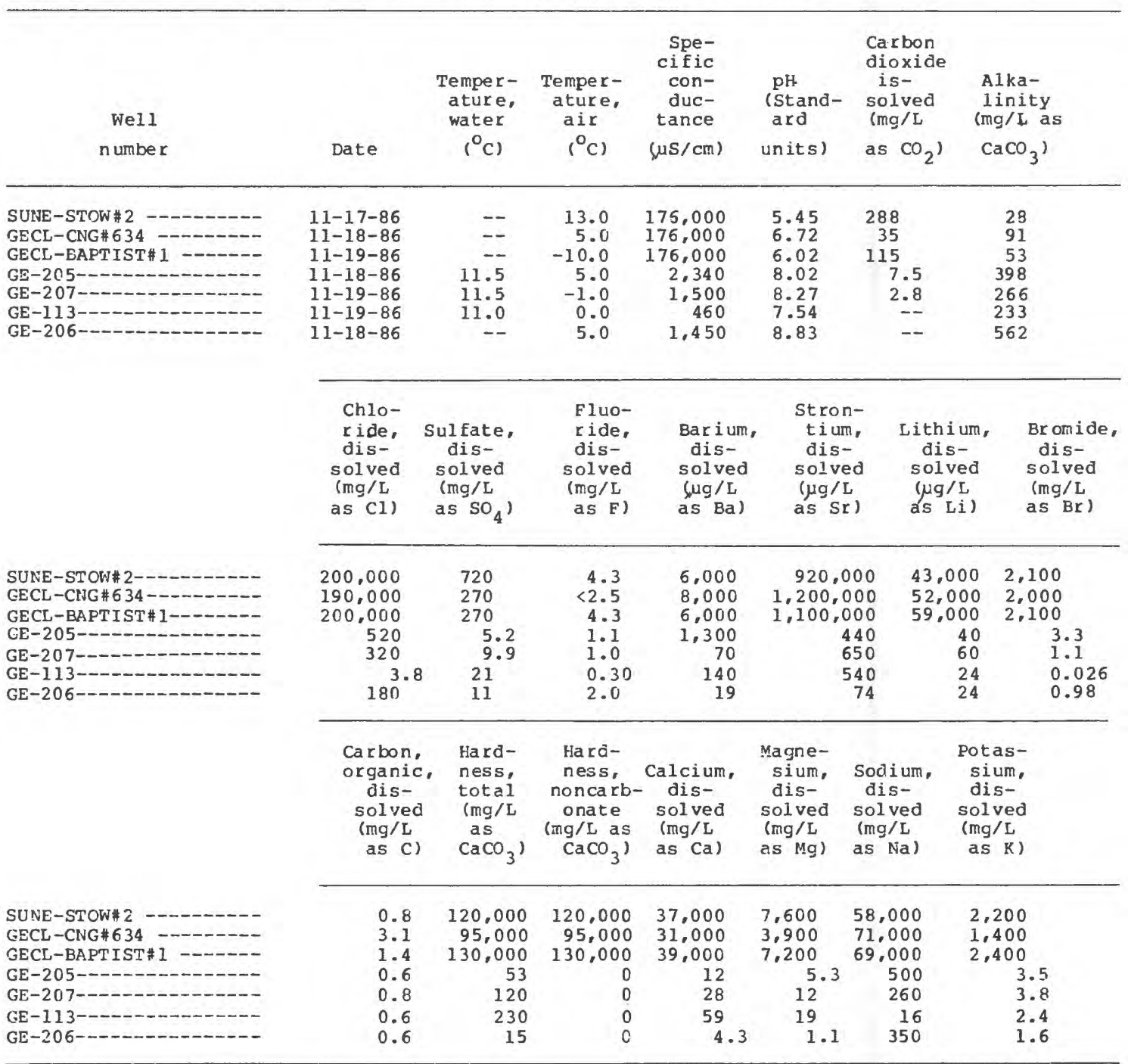




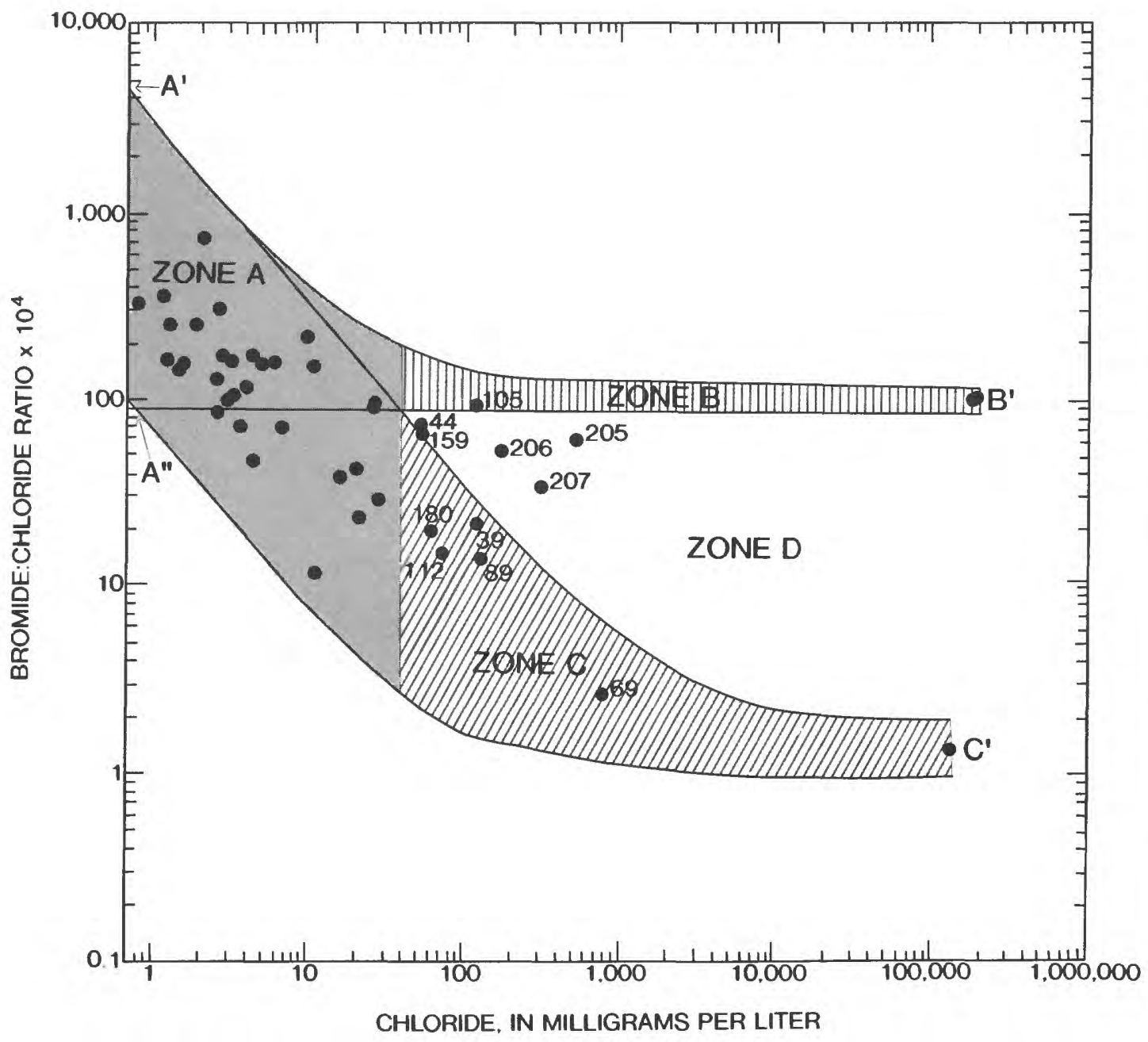

\section{EXPLANATION}

ZONE A--Local ground waters.

ZONE B--Mixtures of local ground waters with local oilfield brines.

VIIIIID

ZONE C--Mixtures of local ground waters with local halite-solution brines.

ZONE D--Mixtures of local ground waters with local oilfield brines and local halite-solution brines, or other natural formation fluids.

A" POINTS DISCUSSED IN TEXT

-69 WELL AND WELL NUMBER--The county code prefixes, GE-, have been omitted from well numbers.

Figure 25.--Bromide:chloride ratios for local ground waters and brine-mixing zones 
have been affected by halite solution (C. J. Khourey, Ohio Environmental Protection Agency, written commun., 1988). The water from well GE-105 plots within zone B, which indicates that it is a mixture of local ground water and oilfield brine. This interpretation is supported by the increase in TOC in this well in 1986 (fig. 24).

The waters from wells GE-44, GE-159, GE-205, GE-206, and GE-207 plot within zone $\mathrm{D}$, which suggests that they may be a mixture of local ground water with both oilfield brine and halite-solution brine, or with other local, natural-formation waters. Wells GE-205, GE-206, and GE-207 were sampled because of homeowner complaints of brine contamination registered with the Division of Oil and Gas of the Ohio Department of Natural Resources. Well GE-44 is completed in the Bedford Shale along its outcrop in the northern part of the County. Well GE-159 is completed in the Berea Sandstone in the central part of a regional flow cell. The origin of these waters is problematic but may represent slight differences in formation-water chemistry or the influence of brine contamination.

Similar interpretations were made by Knuth and others (1990) in a study from Summit County, Ohio, in which they used $\mathrm{Br}: \mathrm{Cl}$ ratios for two samples of Clinton zone brine of 0.0114 and 0.0117 -quite close to the ratio of 0.0105 used herein. In addition, the $\mathrm{Br}: \mathrm{Cl}$ ratios for freshwater in their study lie within the fresh-water ratios computed for waters in Geauga County, indicating good consistency in the data.

\section{SIMULATED GROUND-WATER FLOW}

A steady-state, three-dimensional ground-water-flow model was constructed using the finite-difference ground-water-flow program of McDonald and Harbaugh (1984). Results of the model were used to improve understanding of the geohydrologic conditions within the study area to aid county planners in making decisions that may affect the ground-water flow system. Specifically, model results were used to determine groundwater flow paths and to estimate the length of time a particle of water may reside in the aquifers and regional confining unit before reaching local discharge points. Such information will help county planners understand the advective transport of contaminants (contaminants that travel with the average velocity of the bulk fluid) within the groundwater flow system. In addition, the ground-water-flow model was used to predict the response of the flow system to anticipated additional ground-water development as a result of forecasted population growth. All predictions were based on the assumption that the ground-water flow system would eventually reach a new steady-state condition after development. Effects of commercial and industrial growth on the flow system were not addressed by this study. 


\section{Description of the Model}

\section{Assumptions}

The following simplifications and assumptions were made to simulate the groundwater-flow system:

(1) There is no flow across the bottom of the aquifer in the Berea Sandstone.

(2) The buried valley along the southern periphery of the study area is, in part, an area of discharge for water in the Berea Sandstone.

(3) The buried valley underlying the Chagrin River along the southwestern periphery of the study area is not an area of discharge for water in the Berea Sandstone.

(4) All saturated glacial deposits are hydraulically connected and compose a single aquifer throughout the study area.

(5) The hydraulic conductivity of each aquifer or regional confining unit is uniform with depth, but differs spatially.

(6) Ground-water levels in January-February 1986 approximate average annual water levels within the study area.

(7) The ground-water flow system as defined in January-February 1986 is steady state; that is, ground-water levels and velocities at any point within the flow system are constant with time, and the sum of all recharges and discharges to the system are equal.

\section{Discrete Hydrogeologic Framework}

The finite-difference grid used to simulate ground-water flow within the study area is illustrated in figure 26 . The grid covers approximately 530 square miles and coincides with the natural physical boundaries of the ground-water-flow system (fig. 1). Grid spacing is uniform and was chosen to represent the aquifers and confining unit as part of a regional ground-water-flow system; each grid block is $3,500 \mathrm{ft}$ on a side.

The flow system was simulated by three model layers. Model layer 1, the top layer, represents the unconfined surficial aquifer in the Pottsville Formation or the glacial deposits where the Pottsville Formation is discontinuous. In general, model layer 2 


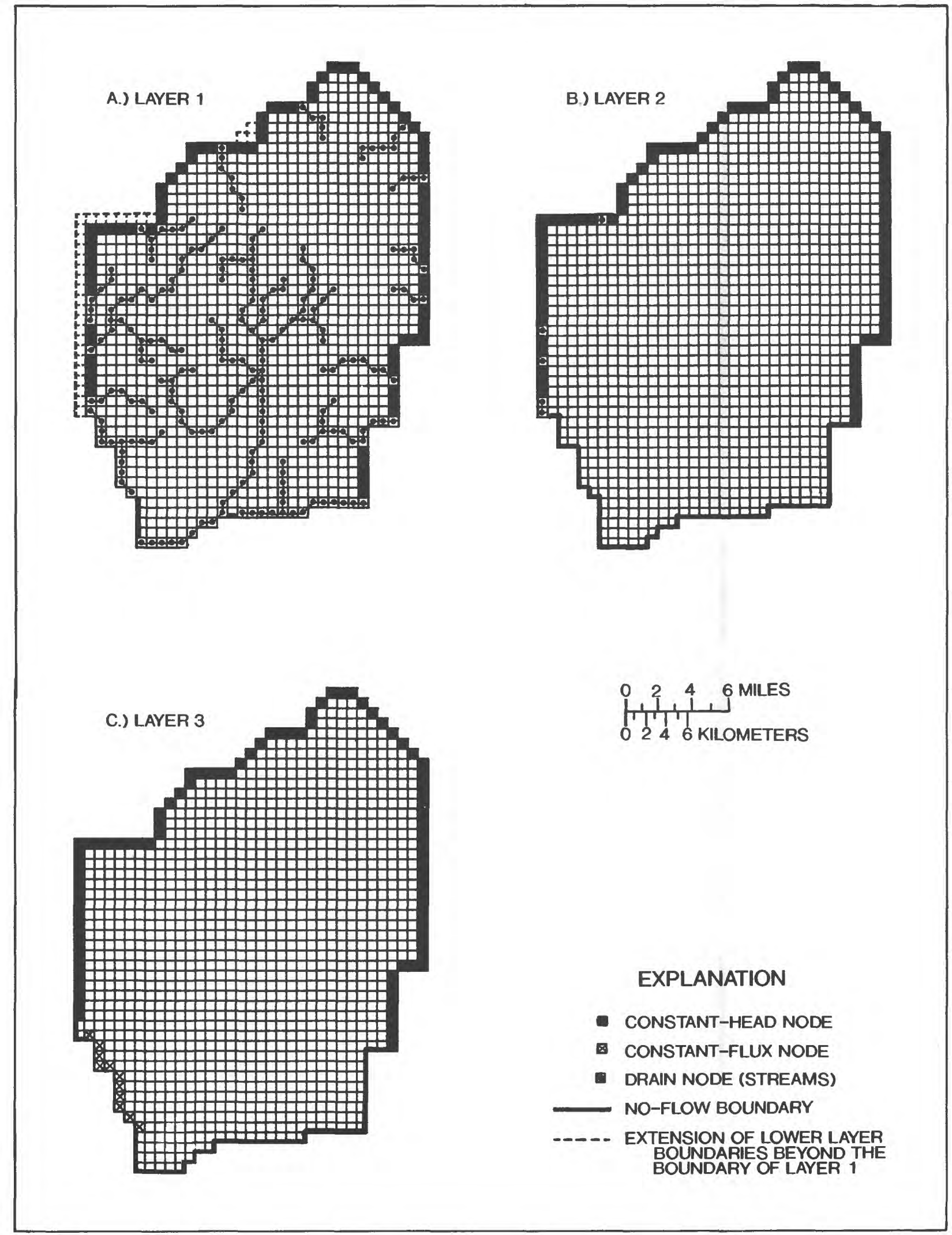

Figure 26.--Boundaries used to simulate the ground-water-flow system represented by A.) layer $1, B$.) layer 2 , and C.) layer 3. 
represents the Cuyahoga Group as a single confining unit, and model layer 3 represents the Berea Sandstone as a confined aquifer. Where buried valleys cut through the Cuyahoga Group and the Berea Sandstone, water-transmitting properties associated with the glacial deposits are simulated.

\section{Boundary Conditions}

The upper boundary of the model incorporates several hydrologic boundaries. These hydrologic boundaries include the water table, simulated as a free-surface boundary, and the Cuyahoga, Chagrin, and Grand Rivers, their tributaries, and numerous lakes and reservoirs, simulated as head-dependent flux boundaries. Because the October 1980 streamflow gain/loss study indicated that all streams within Geauga County gain water from the ground-water-flow system, the streams were represented in the model as drain nodes (fig. 26). The use of drain nodes allowed water to flow into but not out of the simulated streams. Drain, or streambed, elevations were taken from U.S. Geological Survey topographic maps.

The lower boundary of the model coincides with the bottom of the Berea Sandstone. In Geauga County, the Berea Sandstone is underlain by the Bedford Shale, which is notably unproductive as a source of ground water throughout most of the County (Baker, 1964). It is assumed there is no vertical flow between the Berea Sandstone and the Bedford Shale; consequently, the lower boundary of the ground-water-flow system was simulated as a no-flow boundary.

In general, the northern, eastern, and western boundaries of the model coincide with the bedrock outcrops illustrated in plates 1 and 2 . Ground water can be seen seeping out along these outcrops within the study area. The January-February 1986 water-level surfaces in figures 6 and 8 also indicate that ground-water flow is outward across these bedrock boundaries. As a result, the northern, eastern, and western boundaries of the model were simulated as specified-head boundaries (fig. 26) by use of constant-head nodes. The values assigned to the constant-head nodes correspond to the altitudes of the ground-water seeps along the bedrock outcrops.

The southern boundary of the model coincides with the buried valley along the southern periphery of the study area (fig. 3). Various types of information were used to determine the nature of this flow-system boundary. The Cuyahoga River, in part, flows across the highly permeable glacial deposits in this buried valley. The October 1980 gain/loss study indicated that the Cuyahoga River gains water from the local groundwater-flow system. The gain/loss study also indicated that the Chagrin River, whose headwaters originate along this buried valley, gains water from the local ground-waterflow system. In addition, the headwaters of Eagle Creek (fig. 1) originate along the buried valley. Although no gain/loss data are available for this section of Eagle Creek, 
the Ohio Drilling Company (1971) noted anomalously large dry-season flow in Eagle Creek. On the basis of this information, Eagle Creek also is presumed to be a discharge area for the local ground-water-flow system.

Ground-water discharge to these streams along the southern boundary was simulated by assigning drains to the model nodes of layer 1 that correspond to the Cuyahoga River, Chagrin River, and Eagle Creek (fig. 26). The buried valley along this boundary, in part, also dissects the Cuyahoga Group and the Berea Sandstone, which are represented by model layers 2 and 3. It is assumed that the direction of ground-water flow in the Cuyahoga Group along this buried valley is nearly vertical as it is throughout most of the study area. In addition, it is thought that water in the Berea Sandstone discharges into the permeable buried-valley deposits and flows within the valley parallel to the southern boundary of the modeled area or discharges into the overlying streams. As a result, the southern boundaries in layers 2 and 3 were simulated as no-flow boundaries. Because the buried valley is at least $4 \mathrm{mi}$ (six grid blocks) south of Geauga County, these assumptions should not affect model predictions within the County.

The southwestern boundary in layer 3 was simulated as a constant-flux boundary (fig. 26) because the available bedrock-geologic map (pl. 1) indicates that the buried valley underlying the Chagrin River in this area does not dissect the Berea Sandstone. Therefore, it is assumed that ground water in the Berea Sandstone flows across the boundary and does not discharge at this point (fig. 26). Constant-flux values were calculated by use of Darcy's Law. In these calculations, the value for horizontal hydraulic conductivity was obtained from Rau (1969), and hydraulic gradients were determined from the January-February 1986 potentiometric-surface map of the Berea Sandstone (fig. 8).

\section{Parameter Values}

Parameters necessary to simulate the steady-state ground-water-flow system include horizontal hydraulic conductivity or transmissivity, vertical hydraulic conductivity, recharge, pumpage, and a conductance term for the drains used to simulate the streams. Initial values for these parameters were derived from a number of sources.

The spatial distribution of horizontal hydraulic conductivity that provided the best match between measured and simulated ground-water levels for each model layer is illustrated in figure 27 . The horizontal hydraulic conductivities presented by private sector consultants and U.S. Geological Survey personnel, discussed previously in this report, were used as initial values in the model, whereas the values in figure 27 are the result of trial-and-error adjustment of these initial values during model calibration. These 


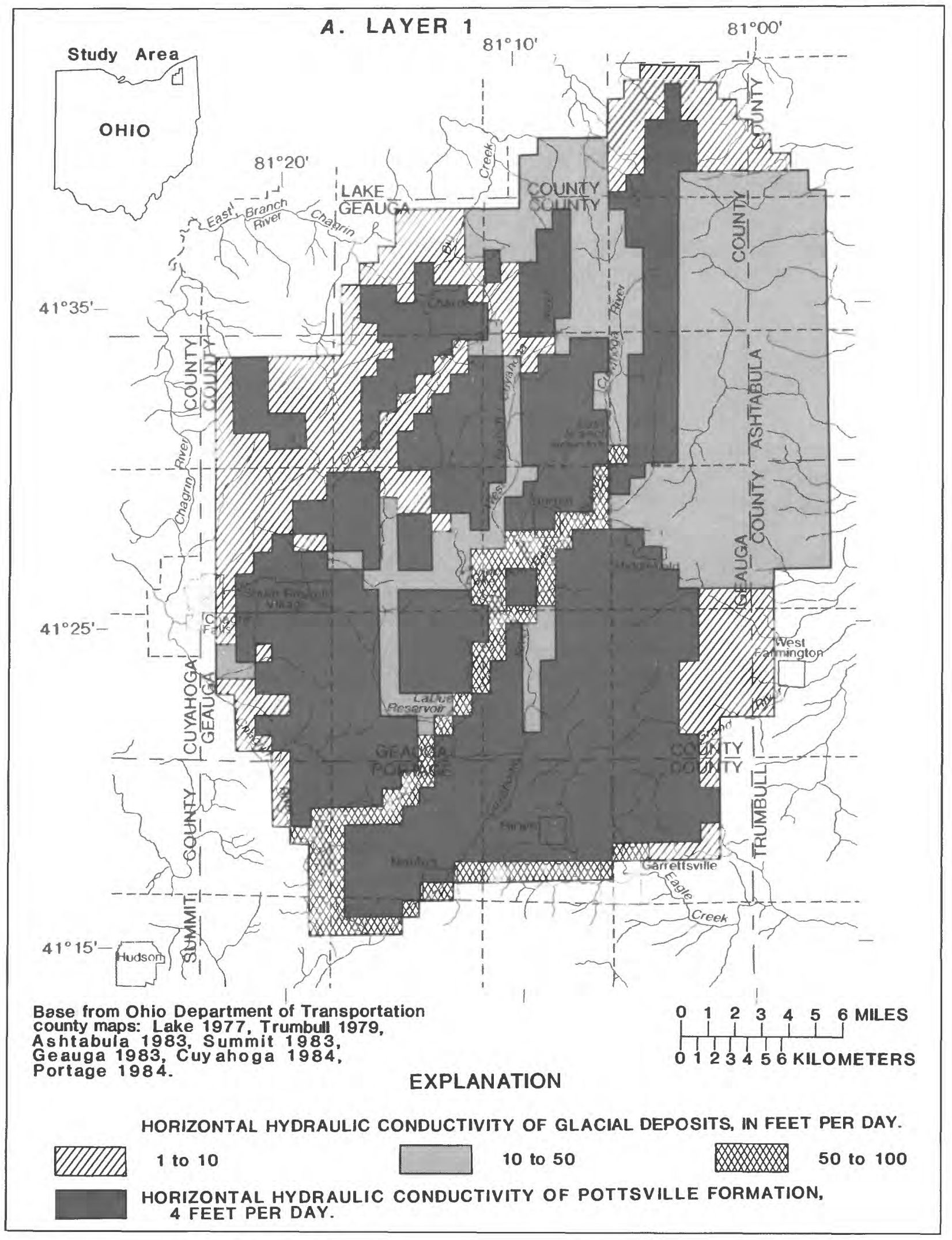

Figure 27.--Horizontal hydraulic-conductivity values used to simulate the ground-water-flow system represented by $(A)$ layer $1,(B)$ layer 2 , and $(C)$ layer 3. 


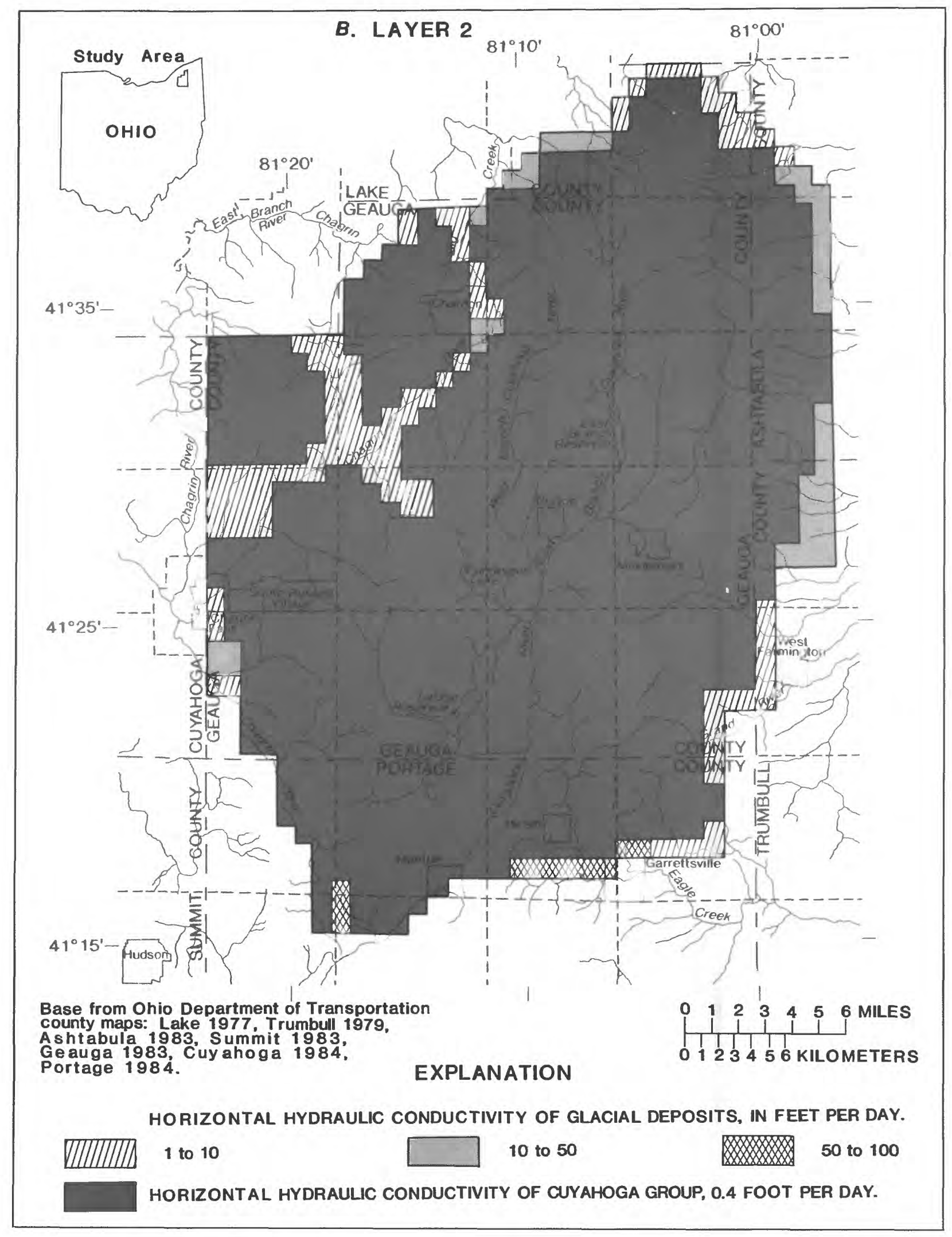

Figure 27.--Horizontal hydraulic-conductivity values used to simulate the ground-water-flow system represented by $(A)$ layer $\left.1, B_{B}\right)$ layer 2 , and $(C)$ layer 3 -Continued. 


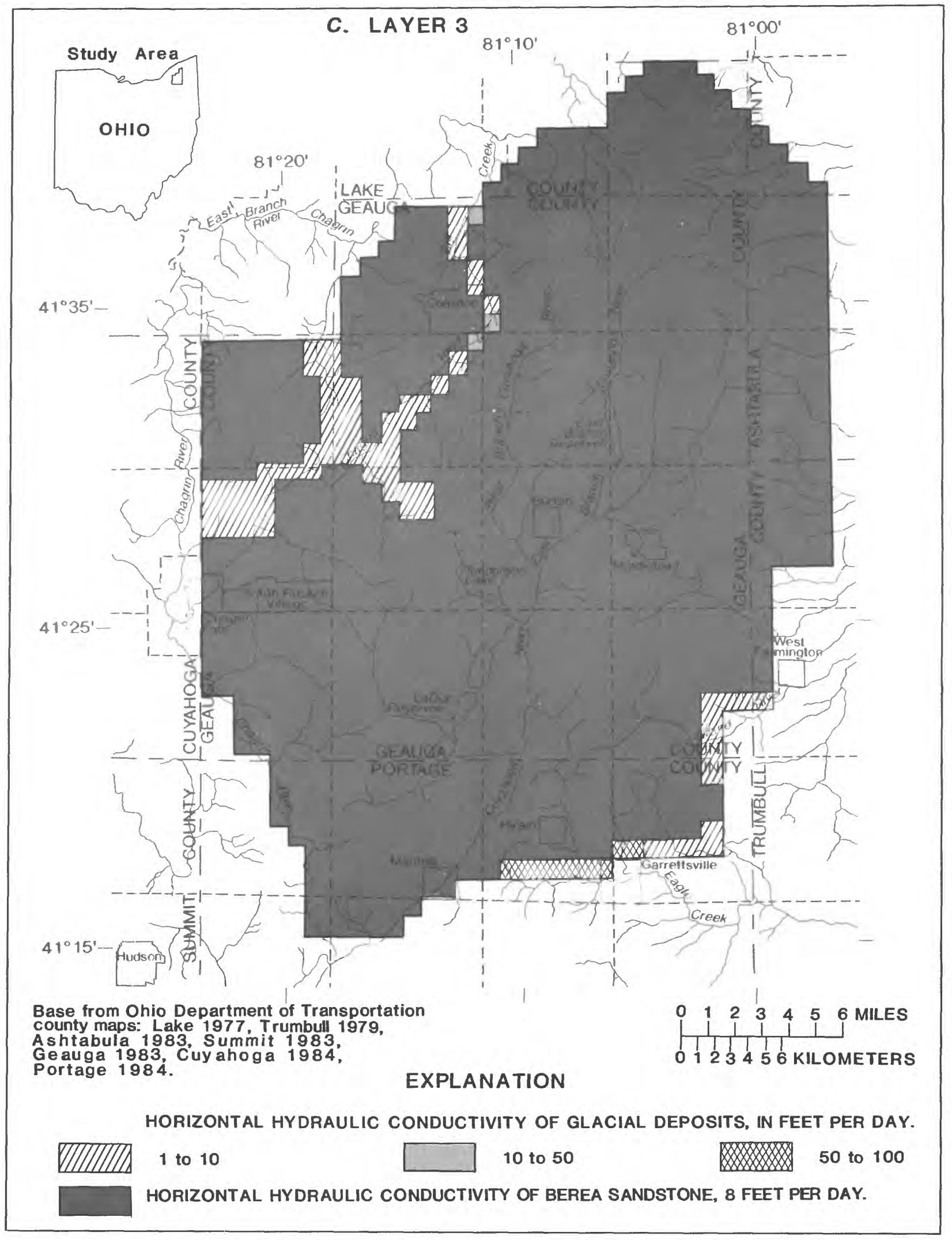

Figure 27.-- Horizontal hydraulic-conductivity values used to simulate the ground-water-flow system represented by $(A)$ layer 1 . $(B)$ layer 2 , and $(C)$ layer 3--Continued. 
horizontal hydraulic conductivity values were multiplied by appropriate saturated thicknesses to determine transmissivity values. Saturated thicknesses were not adjusted during model calibration.

Most glacial deposits were simulated with values for horizontal hydraulic conductivity between 1 and $50 \mathrm{ft} / \mathrm{d}$. The most permeable glacial deposits, located in the buried valleys between Burton and northern Portage County and between Garrettsville and Mantua were simulated with values between 50 and $100 \mathrm{ft} / \mathrm{d}$. For areas in which no aquifer-test or specific-capacity data were available, horizontal hydraulic conductivities were assigned by extrapolating values from areas of known horizontal hydraulic conductivity on the basis of drillers' logs and a map prepared by the Ohio Drilling Company (1971) depicting ground-water potential in the buried valleys of northeastern Ohio.

The Pottsville Formation was simulated with a horizontal hydraulic conductivity value of $4 \mathrm{ft} / \mathrm{d}$ (layer 1, fig. 27). This value is near the low end of the range of values previously discussed. A low value was necessary because each horizontal hydraulic conductivity value in the model represents $0.44 \mathrm{mi}^{2}$ (square miles) of the Pottsville Formation, which is composed of a sandstone matrix with secondary porosity, whereas most of the aquifer-test data represent site-specific aquifer characteristics.

A value for horizontal hydraulic conductivity of $0.4 \mathrm{ft} / \mathrm{d}$ provided the best match between measured and simulated ground-water levels in the Cuyahoga Group (layer 2, (fig. 27). This is the same value calculated from specific-capacity data obtained from drillers' logs of wells included in the current study. The horizontal hydraulic conductivity used to simulate the Berea Sandstone was $8 \mathrm{ft} / \mathrm{d}$ (layer 3, fig. 27) and corresponds to the value calculated by Rau (1969) on the basis of specific-capacity data.

Vertical hydraulic conductivity in unconsolidated sediments and sedimentary rocks generally is less than horizontal hydraulic conductivity in the same units. Values used in the model were as low as $1 \times 10^{-4} \mathrm{ft} / \mathrm{d}$, in the Cuyahoga Group, and as high as $1 \mathrm{ft} / \mathrm{d}$, where the buried valley deposits are highly permeable. These values were determined through trial-and-error adjustments during calibration.

In the simulations, recharge from precipitation was applied to the surficial aquifers. Recharge values used in the model range from $4 \mathrm{in} . / \mathrm{yr}$ to $8 \mathrm{in} . / \mathrm{yr}$. These values are similar to the values calculated by Pettyjohn and Henning (1979) by use of base-flow separation of stream hydrographs. Because pumping from the aquifers intercepts ground water that otherwise would discharge naturally to the streams, these recharge values are less than effective recharge values by an amount equal to the amount of intercepted flow that is consumed. As a result, January-February 1986 domestic pumpage was taken into account in the recharge values used in the model. Pumpage from municipal well fields was formally simulated. In addition, the recharge rates used in the model account for evapotranspiration of ground water. 
The spatial distribution of recharge that provided the best match between simulated and measured ground-water levels is shown in figure 28. Recharge values were distributed on the basis of soil-permeability data (Williams and McCleary, 1982; Ritchie and Reeder, 1979; Reeder and Riemenschneider, 1973; Williams, 1986; and Ritchie and others, 1978).

Drain conductance is a lumped parameter used in the numerical model to describe all of the hydraulic-head loss between the aquifer and the drain (stream). Data needed to calculate drain conductances were not available. Therefore, drain conductances were adjusted during model calibration in order to match measured and model-calculated discharges.

\section{Steady-State Calibration}

The model was calibrated against steady-state conditions assumed to exist in January-February 1986. This was done because January-February 1986 is the only time that ground-water levels were measured in Geauga County and outside the County near the physical boundaries of the ground-water-flow system. It is assumed that January-February 1986 ground-water levels approximate average annual ground-water levels. This assumption is reasonable because the model is regional in nature, the potentiometricsurface maps constructed from observed ground-water-level data have a $50-\mathrm{ft}$ contour interval, and ground-water levels fluctuated less than $10 \mathrm{ft}$ in more than 95 percent of the wells measured during the 1986 water year. The periodic water-level data from the 48 wells measured during the current study and the two previous USGS studies support the assumption of long-term steady-state conditions within the ground-water-flow system.

A comparison of the January-February 1986 simulated contours with measured ground-water levels is shown in figures 29 and 30. Simulated and measured groundwater levels are similar in most instances. More importantly, all major features of the January-February 1986 water-level surfaces, such as the ground-water mounds in figures 6 and 8 , are represented by the simulated contours in figures 29 and 30. Some of the finer details of the ground-water-flow system cannot be approximated with the model because the grid chosen to simulate the regional flow system is too coarse to represent locally steep hydraulic gradients. As a result, simulated and measured ground-water levels differ in some instances. Simulated contours are not presented for model layer 2, which represents the Cuyahoga Group, because of the large vertical-flow component in the Cuyahoga Group.

Data collected during the October 1980 gain/loss study of the Cuyahoga, Chagrin, and Grand Rivers and all their tributaries within Geauga County also were used to calibrate the model. As previously discussed, all stream reaches gain water from the aquifers. Simulated stream gains were within \pm 10 percent of measured gains in all instances. This is a reasonable agreement, considering the magnitude of errors associated with streamflow measurements and that conditions within the ground-water-flow system could 


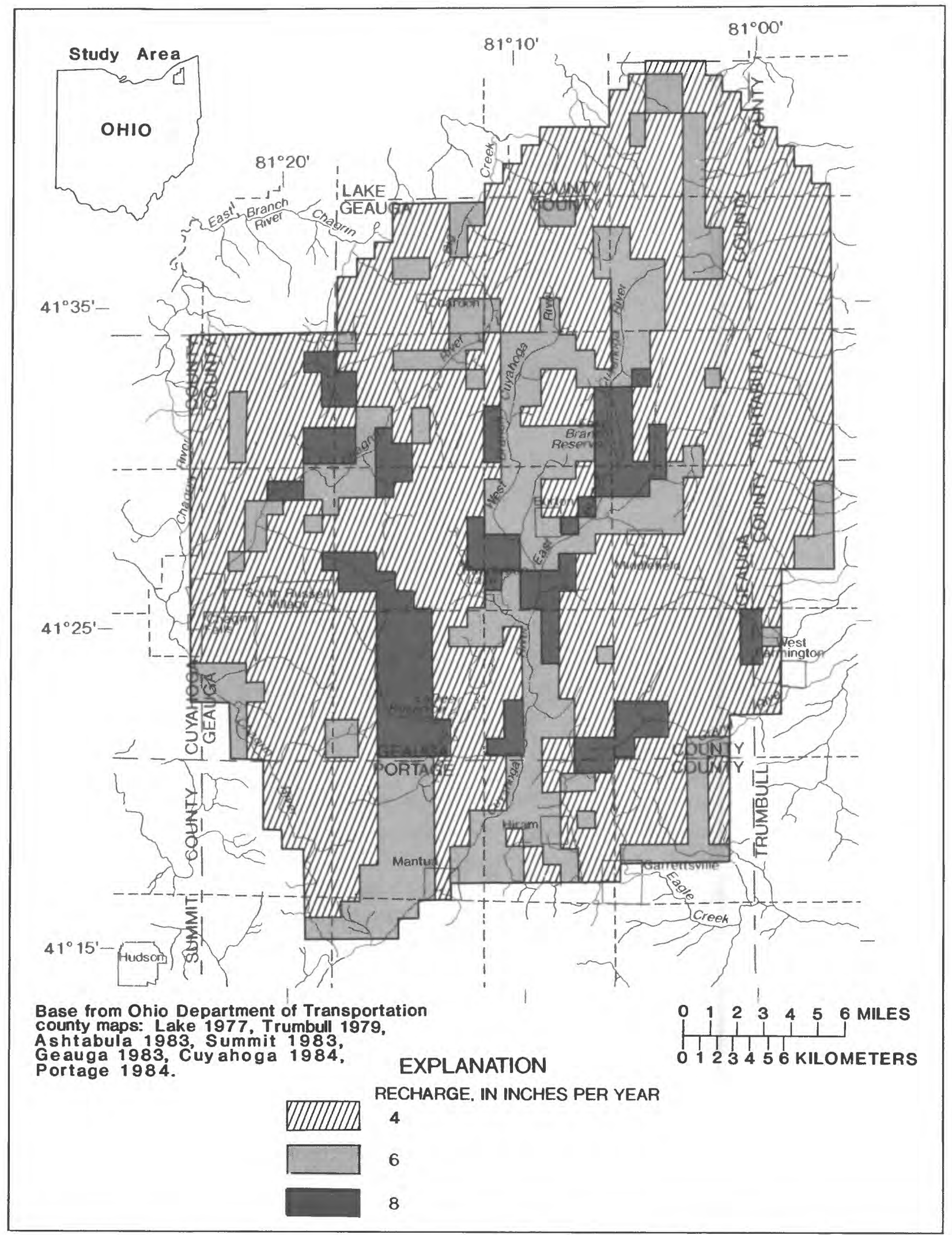

Figure 28.--Spatial distribution of recharge used to simulate the ground-water-flow system. 


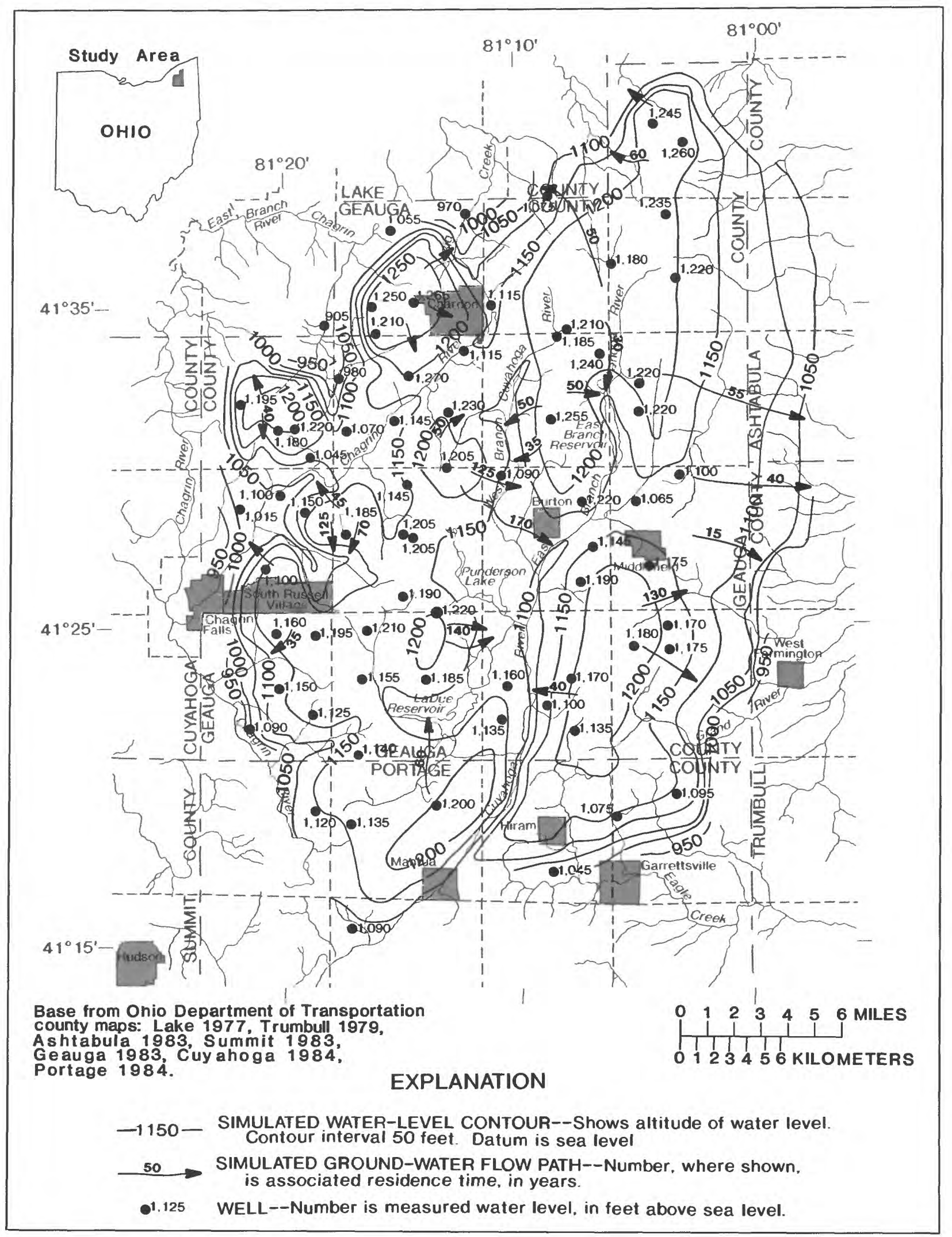

Figure 29.--Simulated ground-water flow paths and residence times, and the relation of simulated contours in layer 1 to ground-water levels measured in January-February 1986. 


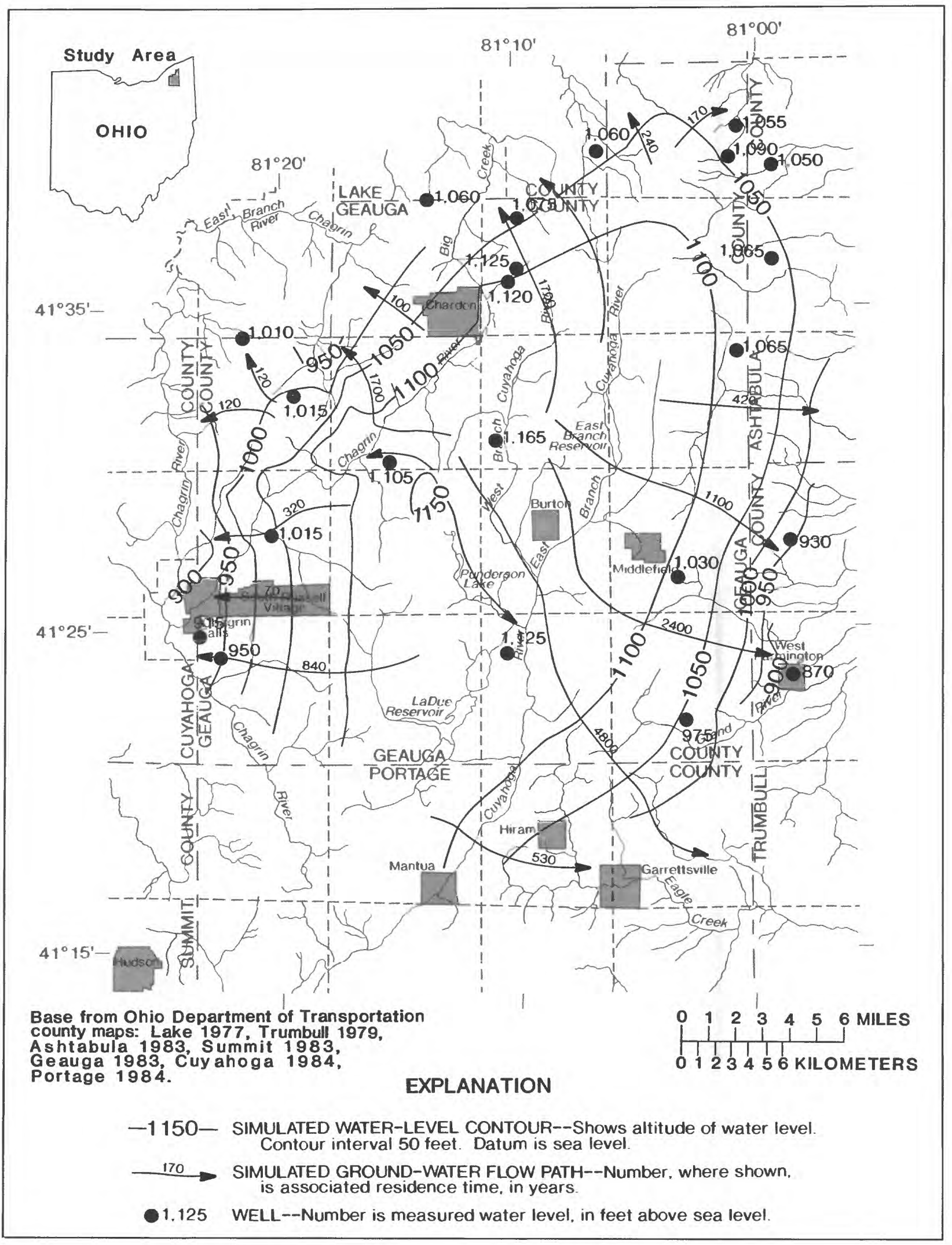

Figure 30--Simulated ground-water flow paths and residence times, and the relation of simulated contours in layer 3 to ground-water levels measured in January-February 1986 
have been slightly different in October 1980 than in January-February 1986 when ground-water levels were measured.

A water budget was calculated using the calibrated steady-state ground-water-flow model (table 6). The budget indicates that precipitation accounts for 100 percent of recharge to the ground-water-flow system within the modeled area. Approximately 69 percent of ground-water discharge is to streams or lakes. Approximately 29 percent of ground-water discharge is flow across the aquifer boundaries, whereas approximately 2 percent of ground-water discharge within the study area is from municipal pumpage.

\section{Ground-Water Flow Paths and Residence Times}

Results of the calibrated steady-state flow model were used in conjunction with a particle-tracking program (Pollock, 1989) to determine ground-water flow paths. Particles of water were placed at select locations at the top of each model layer and tracked to their respective discharge points. Initial particle locations had no significance except to ensure that some local and regional ground-water flow paths were represented. Computed flow paths in figures 29 and 30 are similar to the generalized flow directions drawn

Table 6. - Simulated ground-water budget from the calibrated steady-state ground-water-flow model

\begin{tabular}{clc}
\hline & $\begin{array}{l}\text { Flow } \\
\text { (million }\end{array}$ & \\
$\begin{array}{c}\text { Recharge and } \\
\text { discharges }\end{array}$ & $\begin{array}{l}\text { gallons } \\
\text { per day) }\end{array}$ & $\begin{array}{c}\text { Percentage } \\
\text { of total }\end{array}$ \\
\hline
\end{tabular}

Recharge:

Recharge from precipitation

Total

112

100

Discharges:

Ground-water seepage to

streams and lakes.

Boundary flux

Municipal wells

Total 
perpendicular to lines of equal altitude on the January-February 1986 water-level maps (figs. 6 and 8). Flow in model layer 1 is from bedrock highs toward the lowlands. The Cuyahoga, Chagrin, and Grand Rivers and their tributaries all are discharge areas in model layer 1. Flow in model layer 3 is predominantly toward the northern, eastern, and western boundaries of the model. Some ground water in model layer 2 discharges along the northern, eastern, and western model boundaries; however, most ground-water flow in model layer 2 is vertical. To determine vertical ground-water flow paths, a particle of water was placed at the water table in the center of each model block along rows within the model grid that correspond to geologic sections A-A' and D-D' as illustrated in plates 1 and 2. Figure 31 illustrates the general relation between the ground-water-flow regimes in the various aquifers and the leaky regional confining unit (Cuyahoga Group), as determined by use of the particle-tracking program. It is apparent from this figure that much of the ground water in the Pottsville Formation and the surficial glacial deposits discharges to major streams, their tributaries, and along the exterior boundaries of the ground-water-flow system. It also is apparent from the refraction of the pathlines that the Cuyahoga Group controls vertical ground-water movement within the flow system. In addition, the Berea Sandstone receives most of its recharge through leakage from the overlying aquifers or confining unit.

An infinite number of flow paths and associated residence times can be determined and used to characterize ground-water flow within the study area. As examples of the approximate time a particle of ground water or a conservative chemical contaminant may reside within each aquifer, residence times were calculated for selected flow paths in figures 29,30 , and 31 .

Residence-time calculations were based on the following general equation,

$$
\begin{aligned}
& \mathrm{t}=\mathrm{x} \\
& {\left[\begin{array}{ll}
\frac{\mathrm{K}}{\mathrm{n}_{\mathrm{e}}} & \frac{\mathrm{dh}}{\mathrm{dl}}
\end{array}\right],}
\end{aligned}
$$

where

$t$ is the residence time;

$x$ is the distance traveled within the flow system (L);

$\mathrm{K}$ is the hydraulic conductivity along the flow path $\left(\mathrm{Lt}^{-1}\right)$;

$\mathrm{n}_{\mathrm{e}}$ is the effective porosity; and

$\frac{\mathrm{dh}}{\mathrm{dl}}$ is the hydraulic gradient along the flow path $(\mathrm{L} / \mathrm{L})$. 
This equation describes advective flow only and does not account for the effects of hydrodynamic dispersion (spreading of a liquid substance) on ground-water residence times. The determination of ground-water residence times does not account for the length of time it takes a particle of water to travel from the land surface through the unsaturated zone to the water table.

The calculations were performed by use of the particle-tracking program MODPATH (Pollock, 1989). The distance of travel used in each calculation was the distance from the initial location of the particle to its respective discharge point. The product of horizontal hydraulic conductivity and hydraulic gradient for each model block was derived from information contained in the cell-by-cell flow output of the calibrated steady-state ground-water-flow model. In addition, textbook values for specific yield (Walton, 1970) were used to represent effective-porosity values in the calculations because effective-porosity data for the study area were not available.

Specific yield is the ratio of the volume of water that drains from a saturated rock due to the force of gravity to the total volume of the rock. Because some of the water retained by the rock against gravity drainage would be mobile under saturated conditions, values for specific yield generally are slightly lower than values for effective porosity. As a result, substitution of specific yield for effective porosity in the above equation results in a lower-limit approximation of ground-water residence times.

Approximate ground-water residence times for the flow paths chosen to represent flow in the Pottsville Formation and (or) the glacial deposits (model layer 1) (fig. 29) range from 15 to 170 years. Residence times for flow paths in layer 3 (fig. 30), which represent flow in the Berea Sandstone, range from 70 to 4,800 years. Approximate ground-water residence times for the vertical flow paths labeled in figure 31 range from 15 to 2,200 years. These estimates are not meant to be comprehensive because an infinite number of flow paths and associated residence times can be used to describe groundwater flow within the study area. Rather, they are presented to provide a general approximation of the time that ground water may reside within the flow system.

\section{Effects of Increased Pumpage}

In addition to providing information on ground-water flow paths and residence times, the calibrated model was used to identify areas within Geauga County where ground-water levels may be affected by increased domestic pumpage. Before predictions were made with the model, the constant-head nodes along the model boundaries, discussed previously, were replaced with drain nodes. Drain conductances were adjusted until the model-calculated ground-water levels and boundary fluxes were similar to those calculated by the model with the constant-head nodes. This was done to prevent the constant-head nodes, which allow no ground-water-level fluctuation to occur in the corresponding model-grid blocks, from interfering with the simulation of ground-waterlevel declines near the model boundaries. 

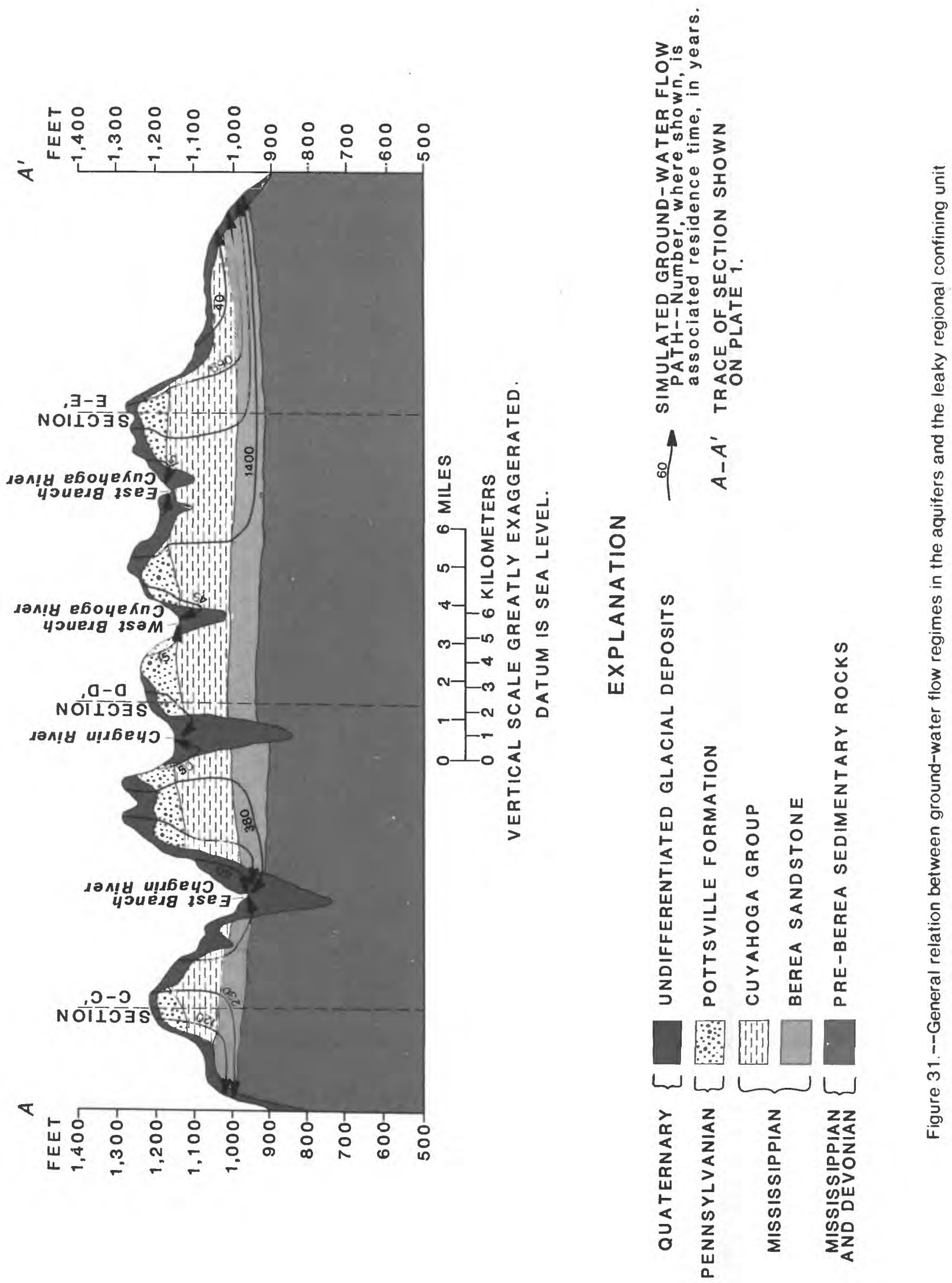

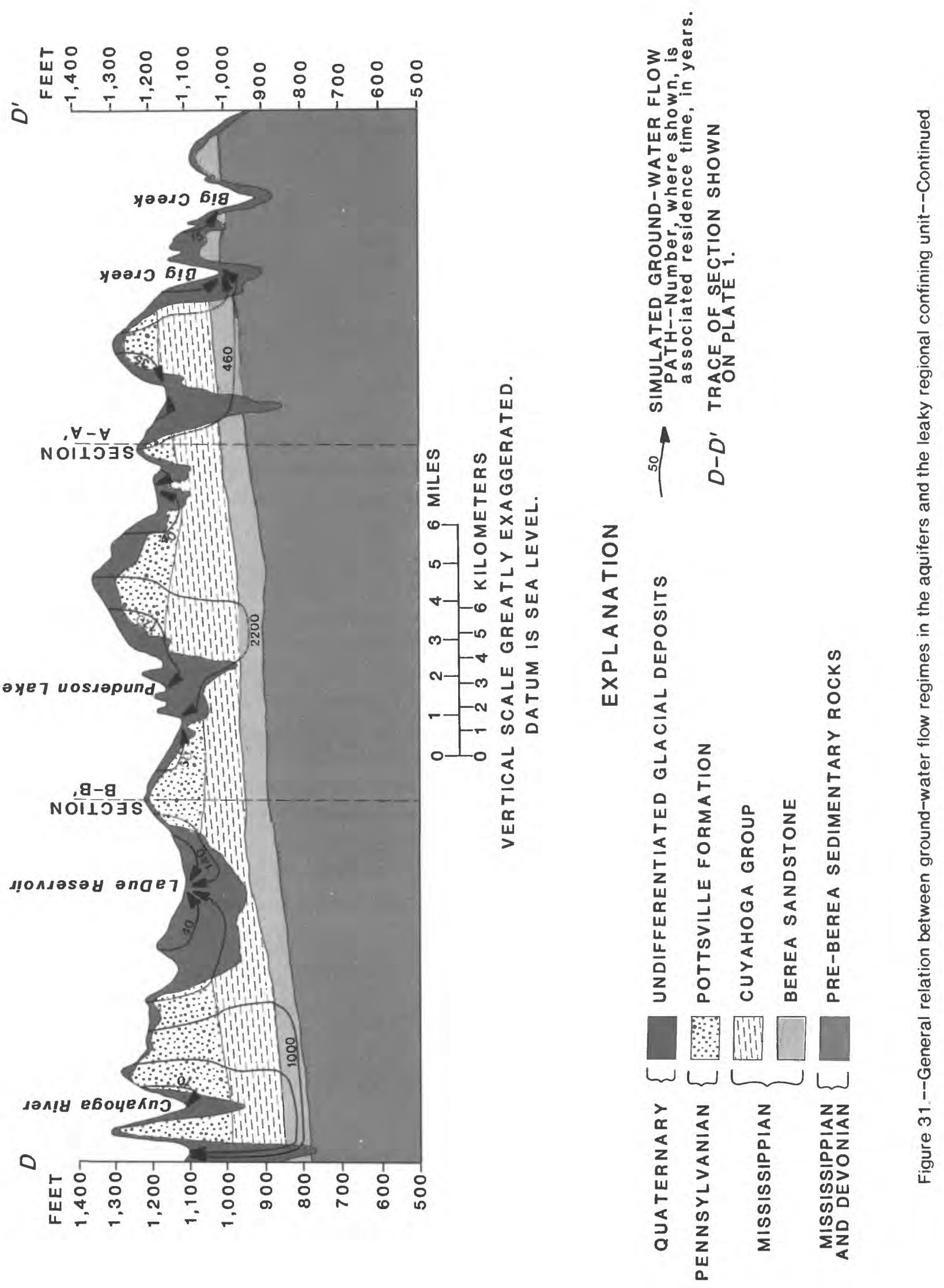
The predictive simulations focus on drawdown in the surficial aquifers (model layer 1) with respect to the steady-state flow conditions that existed in January-February 1986. Changes in discharges to the streams or outflow across the boundaries that would result from increased pumpage are not quantified herein. Estimates of increased domestic pumpage are based on estimates of population growth between the years 1985 and 1995, 1985 and 2000, and 1985 and 2005. It is assumed that the estimate for the 1985 population within Geauga County (Northeast Ohio Area Coordinating Agency, 1983) represents the population within the County in January-February 1986.

All predictive simulations were based on the following additional assumptions: the ground-water-flow system would attain a new steady-state condition after development; all new wells would penetrate fully the aquifer in which they are completed; all new domestic wells would produce water either from the Pottsville Formation or from the glacial deposits (model layer 1); and no water produced from these wells would be returned to the aquifers through septic systems. The final two assumptions allow the predictive simulations to approximate conditions in which the greatest amount of drawdown is likely to occur. In reality, some of the new domestic wells in Geauga County are likely to produce from the Cuyahoga Group and the Berea Sandstone. Some water withdrawn from wells in unsewered areas would be returned to the aquifers through septic systems. The emphasis of the predictive simulations, however, was to identify areas in which ground-water-level declines are most likely to occur, not to predict absolute declines.

The results of three predictive simulations are presented herein. These simulations illustrate the spatial distributions and approximate amounts of ground-water-level decline that could occur if the 1985 population of Geauga County grew by 12,17, and 21 percent, respectively. Results of the first simulation (fig. 32) depict the amount of drawdown that may occur if the population of Geauga County increases 12 percent from a 1985 population of 80,100 to a 1995 population of 90,000 . This does not mean that the amount of drawdown shown in figure 32 would occur by the year 1995; rather, this is the amount of drawdown that eventually could occur, provided all the assumptions are met, if a population of 90,000 is achieved.

Figure 33 is included to help explain the pumpage data used in the first predictive simulation. The shaded blocks represent areas in which no increase in domestic pumpage is expected. These are areas that either are not zoned for residential development or are zoned residential but already are developed completely. The numbers in figure 33 represent the approximate amount of pumpage applied to each block in layer 1 of the model. The exact amount of pumpage applied to each of these blocks was obtained by multiplying the estimated population growth per township by $75 \mathrm{gal} / \mathrm{d}$, the estimated per capita water use, and distributing this pumpage estimate among the blocks representing incompletely developed residential areas within each respective township. 


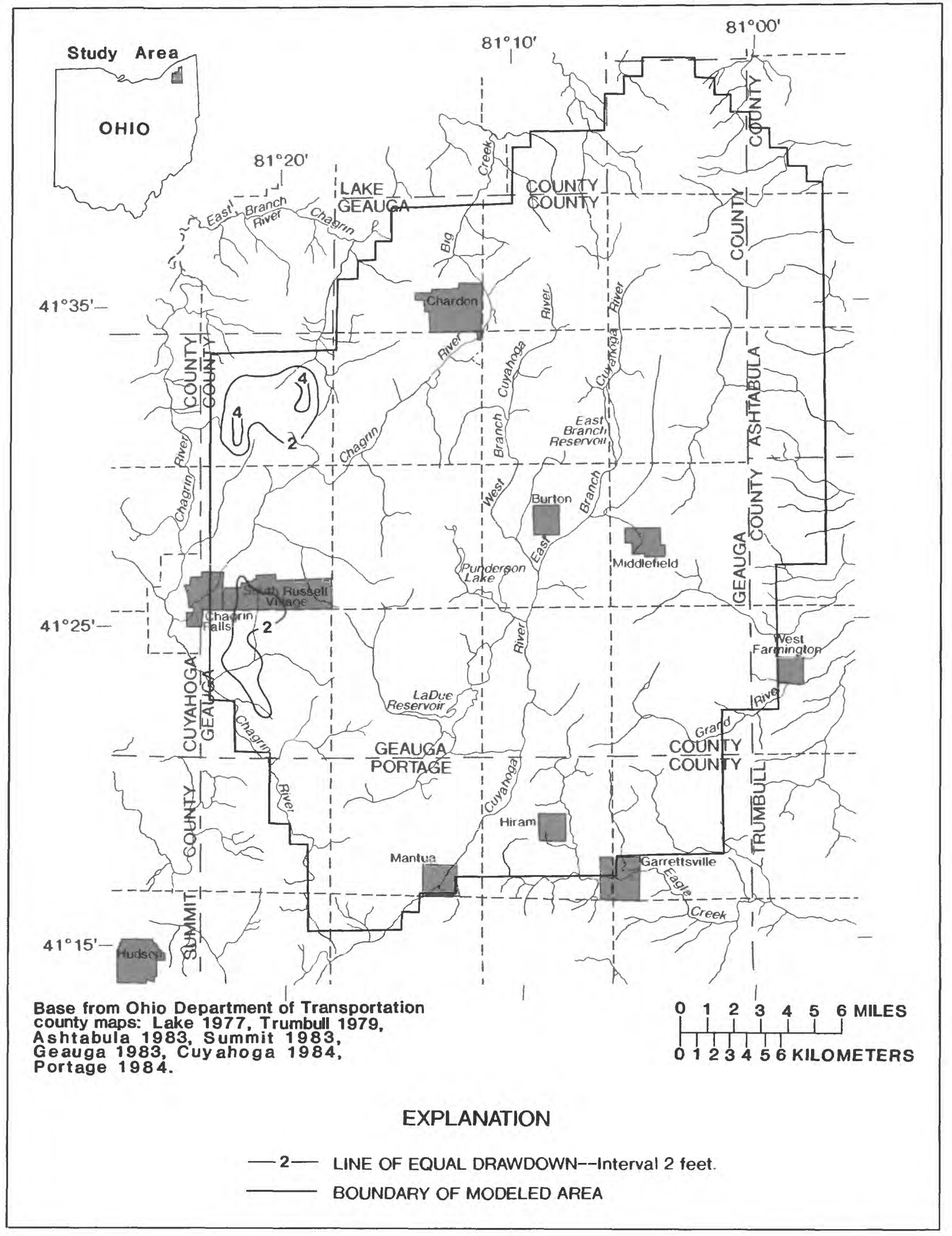

Figure 32.--Amount of drawdown that may occur if the 1985 population of Geauga County increases 12 percent to an expected population of 90.000 in 1995. 


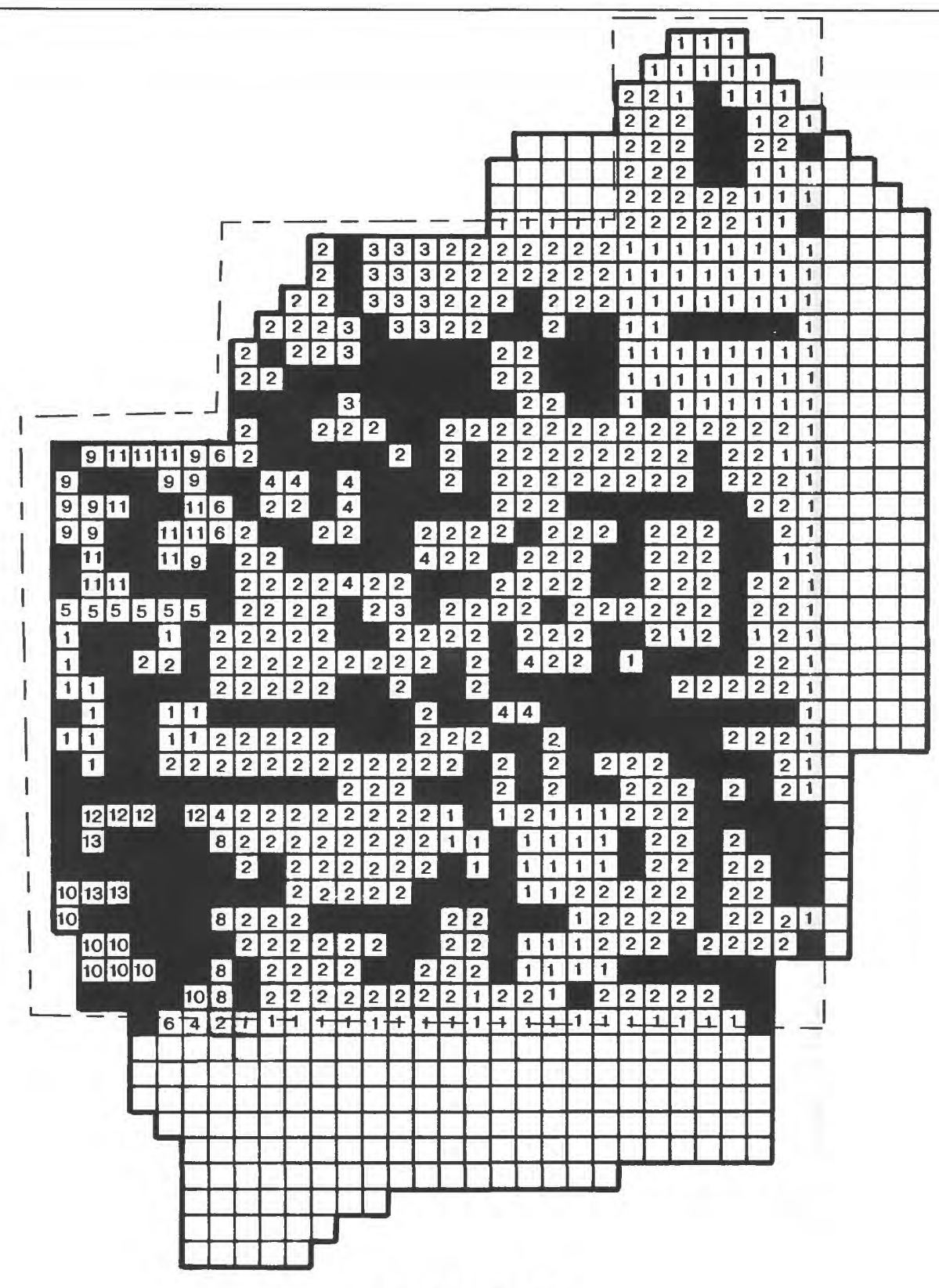

EXPLANATION

- - BOUNDARY OF GEAUGA COUNTY

BOUNDARY OF MODELED AREA

PUMPAGE RATE CODES, IN GALLONS PER DAY

$\begin{array}{lllllll}1 & 0 \text { to } 500 & 6 & 2,501 \text { to } 3,000 & 10 & 6,001 \text { to } 6.500 \\ 2 & 501 \text { to } 1,000 & 7 & 3,001 \text { to } 3,500 & 11 & 6,501 \text { to } 7,000 \\ 3 & 1,001 \text { to } 1,500 & 8 & 3,501 \text { to } 4,000 & 12 & 7,001 \text { to } 7,500 \\ 4 & 1,501 \text { to } 2.000 & 9 & 4,001 \text { to } 4,500 & 13 & 10.001 \text { to } 10,500 \\ 5 & 2,001 \text { to } 2,500 & & & & \end{array}$

Figure 33.--Pumpage data used to simulate the effects of a 12-percent increase in population. 
The areas with notable drawdown in figure 32 correspond to the areas in which the greatest pumpage increases are expected (fig. 33). Figures 34 and 35 illustrate approximate drawdown in the surficial aquifers and expected increase in pumpage between 1985 and 2000, respectively. Figures 36 and 37 present the same information for 1985 to 2005 . In all three simulations, the only notable areas of drawdown occurred in Chester Township, the southern part of Russell Township, South Russell Village, and Bainbridge Township.

None of the wells for which long-term water-level data are available (figs. 9-12) are located within the areas of notable drawdown in figure 32. As a result, it is not known whether these areas already have experienced ground-water-level declines. Again, it is important to note that drawdown should be less than what is depicted in figures 32,34 , and 36 if the assumptions discussed previously are not met.

\section{Limitations of Model Results}

Because a numerical ground-water-flow model can only approximate the physical system it represents, such a model has limitations. As discussed previously, results of the model were used to calculate ground-water residence times. Because this calculation is sensitive to effective-porosity values, and no effective-porosity data were available for the geologic units within Geauga County, textbook specific-yield values were used, and calculated residence times should be viewed as a general approximation of the time ground water may reside within the flow system.

The calibrated model also was used to predict drawdown within the surficial aquifers resulting from an increase in population and a concomitant increase in groundwater pumpage. The model is limited in its utility to predict water-level changes because there were no known data that could be used to verify the time-dependent effects of varied stresses imposed on the ground-water-flow system, and because it provides a solution that represents every $0.44 \mathrm{mi}^{2}$ with a single ground-water level. Actual drawdown near pumped wells could be greater than these simulated average drawdowns. Assumptions that allow the simulations to approximate worst-case scenarios also were made. As a result of these previous conditions, results of the predictive simulations are more useful to identify areas in which drawdown is likely to occur than to determine absolute values of drawdown. This model cannot be used to make site-specific predictions where more refined interpretations are desired. 


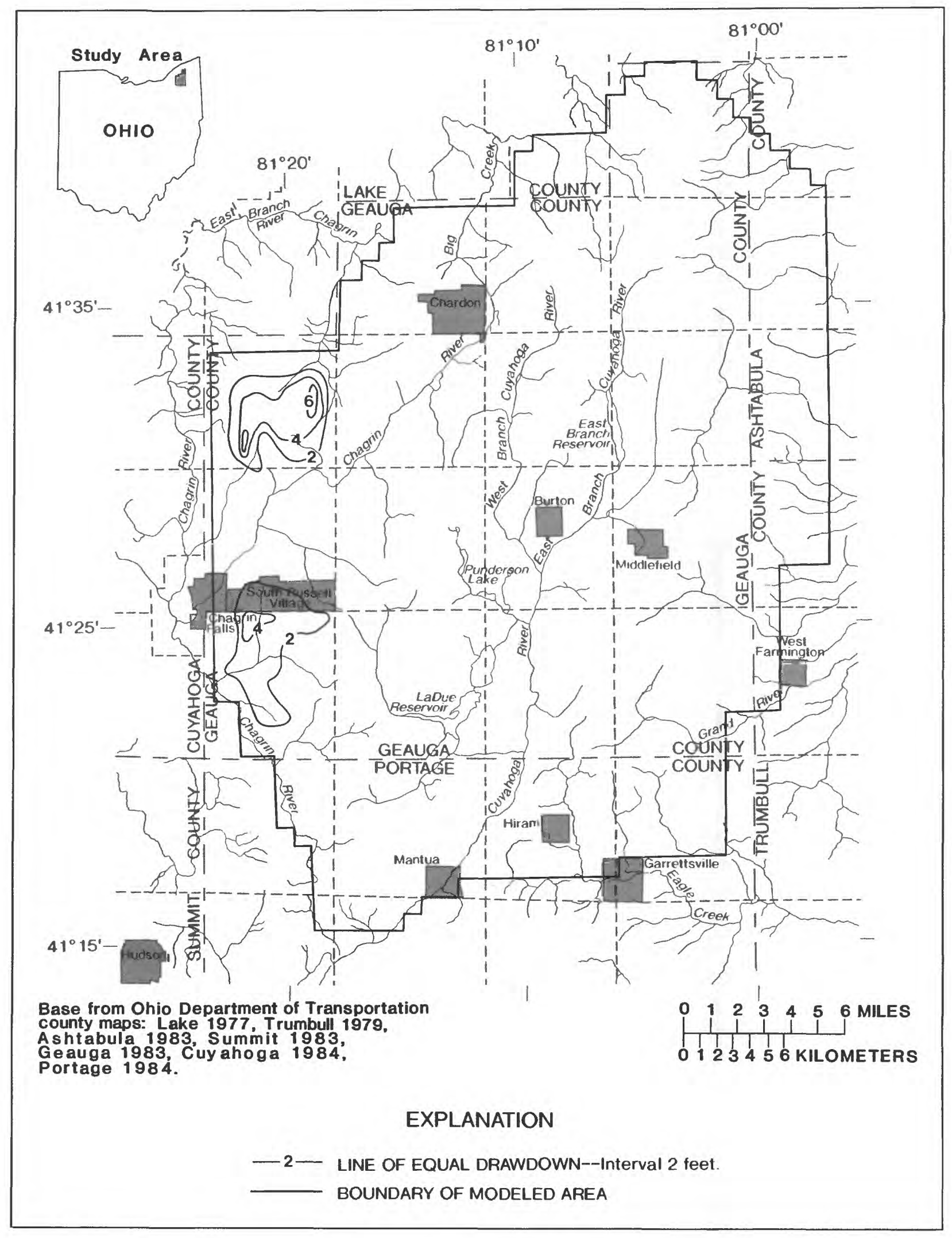

Figure 34.--Amount of drawdown that may occur if the 1985 population of Geauga County increases 17 percent to an expected population of 94,100 in 2000 


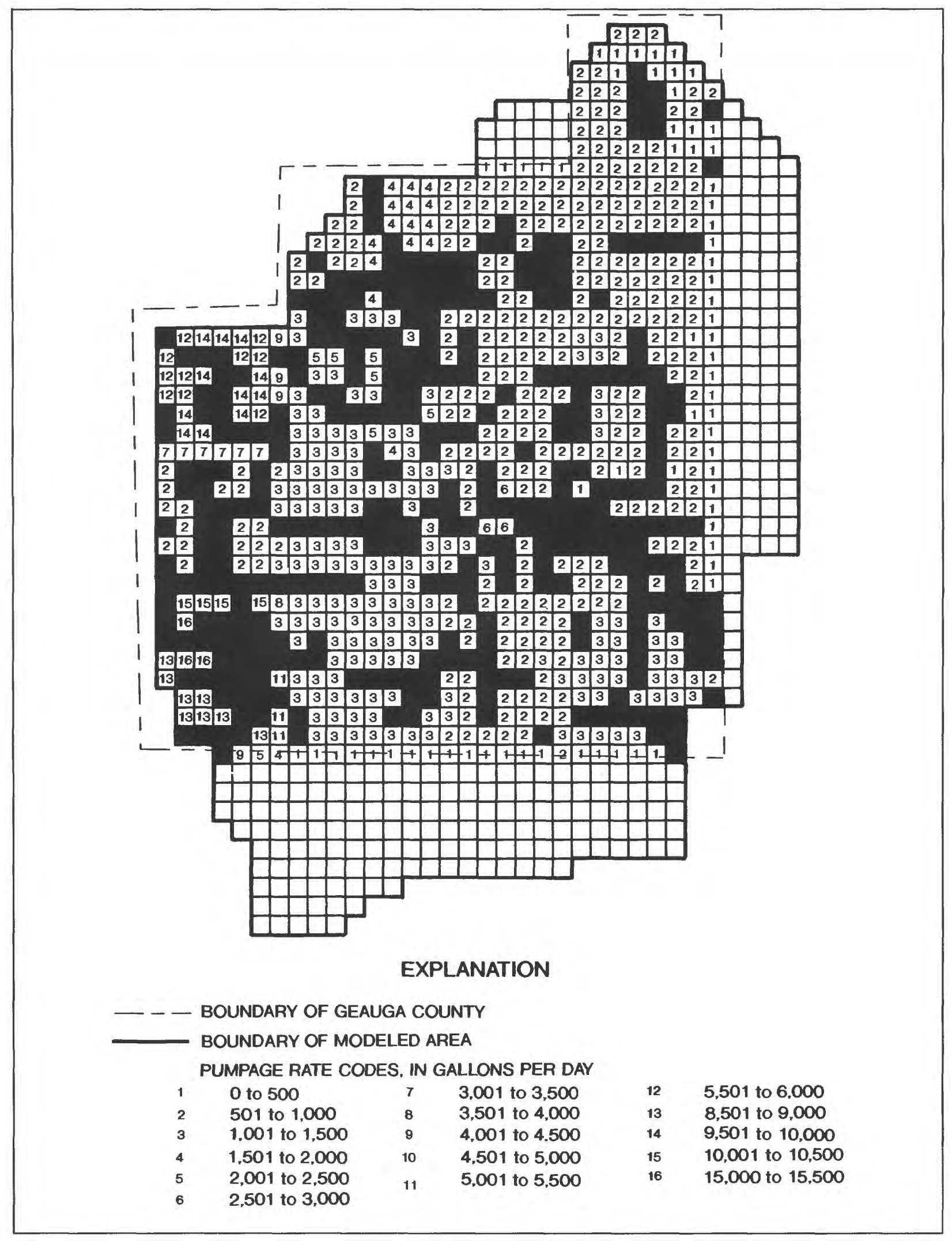

Figure 35.--Pumpage data used to simulate the effects of a 17-percent increase in population. 


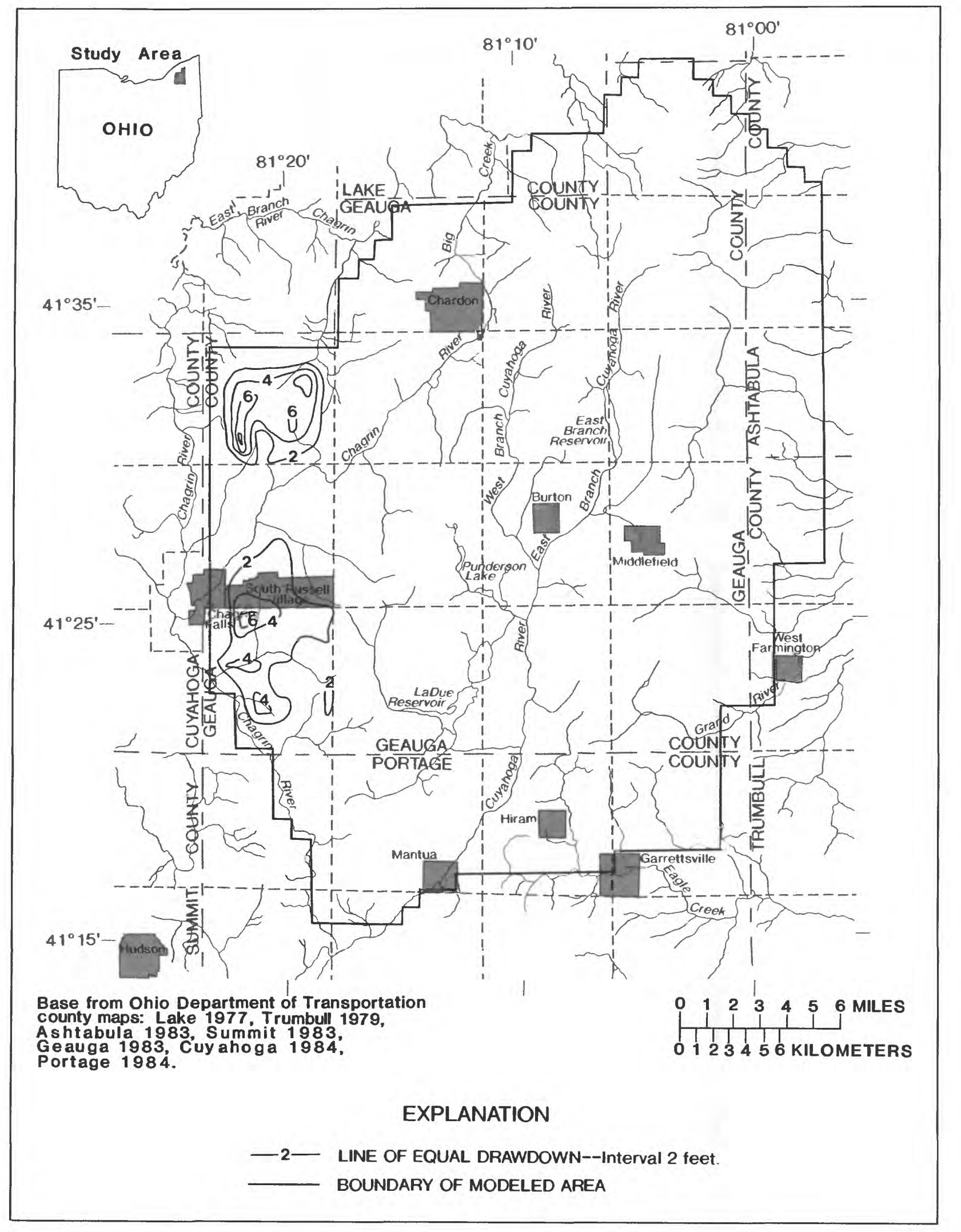

Figure 36--Amount of drawdown that may occur if the 1985 population of Geauga County increases 21 percent to an expected population of 97.200 in 2005 . 


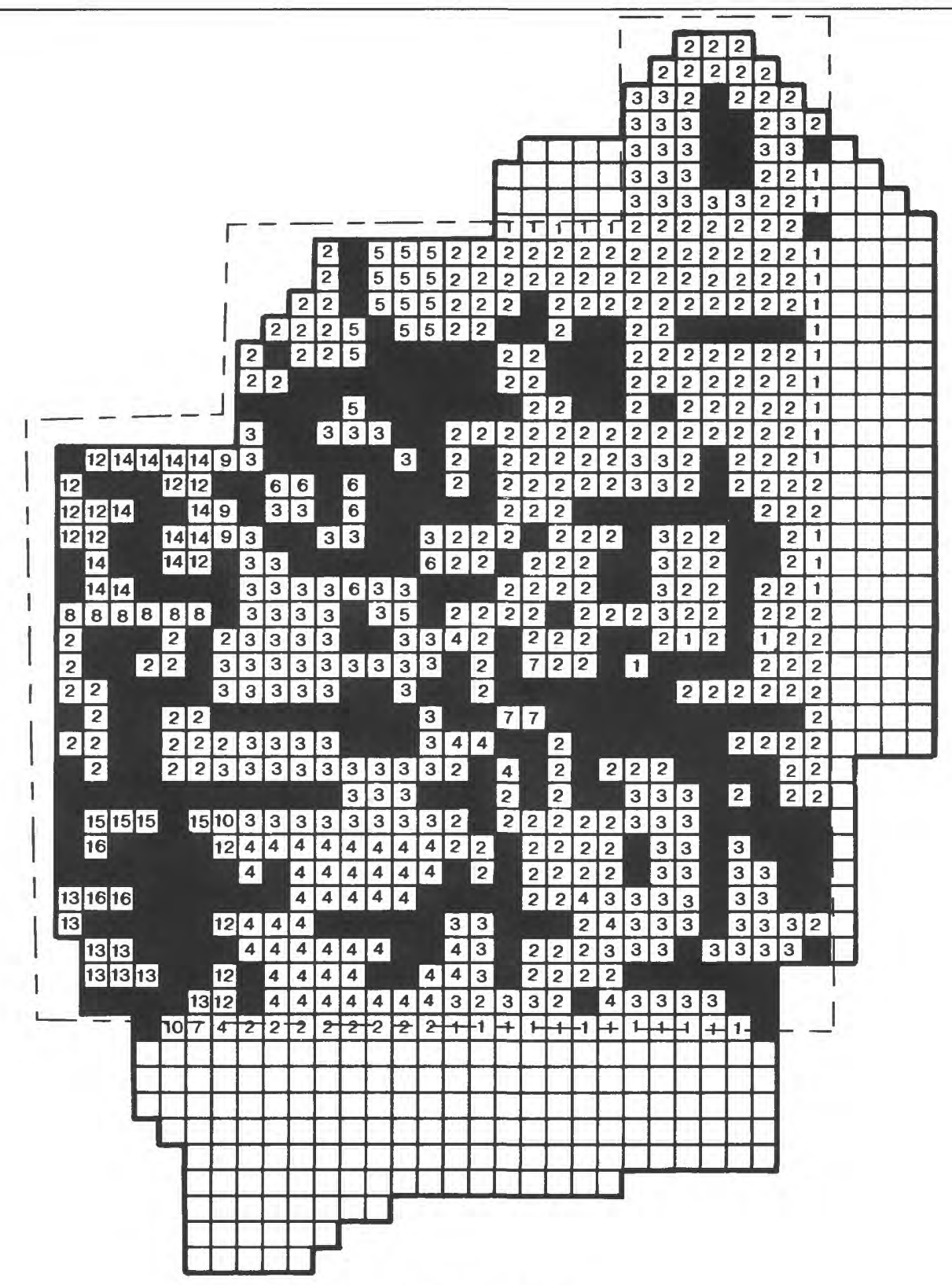

EXPLANATION

- - BOUNDARY OF GEAUGA COUNTY

BOUNDARY OF MODELED AREA

PUMPAGE RATE CODES, IN GALLONS PER DAY

$\begin{array}{lcllll}0 \text { to } 500 & 7 & 3,001 \text { to } 3,500 & 12 & 7,001 \text { to } 7,500 \\ 501 \text { to } 1,000 & 8 & 3,501 \text { to } 4,000 & 13 & 11,001 \text { to } 11,500 \\ 1,001 \text { to } 1,500 & 9 & 4,501 \text { to } 5,000 & 14 & 12,001 \text { to } 12,500 \\ 1,501 \text { to } 2,000 & 10 & 5,001 \text { to } 5,500 & 15 & 13,001 \text { to } 13,500 \\ 2,001 \text { to } 2,500 & 11 & 6,001 \text { to } 6,500 & 16 & 19,001 \text { to } 19,500\end{array}$

Figure 37.--Pumpage data used to simulate the effects of a 21-percent increase in population. 


\section{SUMMARY}

Geauga County is located in the Glaciated Appalachian Plateau physiographic province and has topographic relief of about $500 \mathrm{ft}$ between numerous, isolated highlands and the valley floors of the Cuyahoga, Chagrin, and Grand Rivers. Bedrock generally controls topography within the County, although there are several buried valleys filled with glacial deposits. The thickness of these unconsolidated deposits ranges from zero or a few feet on the bedrock highlands to several hundred feet in the buried valleys. The uppermost bedrock unit exposed in the County is the Pottsville Formation, which varies in thickness and consists in ascending order of four members: the Sharon, the Connoquenessing Sandstone, the Mercer, and the Homewood Sandstone Members. The Pottsville Formation is underlain by the Cuyahoga Group, which consists of interbedded shales and sandstones that range in thickness from 60 to $250 \mathrm{ft}$. The Berea Sandstone underlies the Cuyahoga Group and is about $60 \mathrm{ft}$ thick throughout the County.

Some of the glacial deposits in the County are highly permeable and are commonly used as a source of ground water in the lowlands within the County where the bedrock units are absent. The sandstones in the Pottsville Formation serve as the principal source of ground water within the County. Although some domestic wells obtain sufficient supplies from the Cuyahoga Group, on a regional basis, the shales in the Cuyahoga Group serve as a leaky confining unit between the more permeable materials in the Pottsville Formation and in the underlying Berea Sandstone. The Berea Sandstone is relatively permeable but commonly is not used as a ground-water supply source because of its depth below land surface.

Ground-water levels measured in wells completed in the various geologic units indicate that ground water moves within local flow systems from topographically high recharge areas to topographically low discharge areas. The potentiometric-surface map constructed for the glacial deposits, which commonly are unsaturated on the highlands, and the Pottsville Formation indicates that ground water flows radially away from the highlands toward adjacent streams and buried valleys. Ground-water flow in the Cuyahoga Group predominantly is downward beneath the upland areas. Because it is less commonly cut by buried valleys and stream valleys, ground-water flow in the Berea Sandstone is considered more regional in character than in the overlying geologic units. The potentiometric-surface map of the Berea Sandstone indicates a general, radial-flow pattern away from the upland areas in the central part of the County.

Comparison of measured water levels in the various hydrostratigraphic units shows that, beneath upland areas, ground water flows downward from the glacial deposits and Pottsville Formation across the Cuyahoga Group and into the Berea Sandstone, whereas ground water flows upward beneath major surface-water drainages and within the buried valleys from bedrock units into the glacial deposits. 
Comparison of water levels measured in 1978, 1979, 1980, 1985, and 1986 indicates that no long-term regional water-level changes have occurred in the County. Measured short-term seasonal fluctuations in the various geologic units range in amplitude from several feet in the glacial deposits and the Pottsville Formation, which commonly receive recharge directly from infiltration of precipitation, to $20 \mathrm{ft}$ or more in the Cuyahoga Group and the Berea Sandstone, which receive limited recharge from downward leakage of ground water.

Specific-conductance and TDS data were used to develop the following regression equation for estimating the TDS concentration of ground waters in Geauga County,

$$
\mathrm{TDS}=0.670(\mathrm{SpC})-24.8,
$$

where TDS is in milligrams per liter and $\mathrm{SpC}$ is in microsiemens per centimeter at 25 degrees Celsius and should be limited to values ranging from 150 to $1,000 \mathrm{uS} / \mathrm{cm}$.

Ground-water samples collected from 38 wells were used to determine stratigraphic, regional, and temporal changes in ground-water quality within the County. These data indicate that most ground waters in the surficial glacial deposits and the Pottsville Formation are a calcium bicarbonate-type, whereas most ground waters in the Cuyahoga Group are a sodium-bicarbonate type. The chemical character of ground waters from the Berea Sandstone is highly variable in terms of cations. Some waters in the Berea Sandstone are calcium bicarbonate-types, others are sodium bicarbonate-types, and others are mixedcation bicarbonate types.

The change in dominant cation character from calcium to sodium between the surficial aquifers and the Cuyahoga Group, and the associated decrease in total hardness and increase in TDS, is believed to be caused by cation exchange in clays and concomitant solution of carbonate minerals. The exchange process occurs naturally whereby calcium ions in downward-moving ground water from the overlying surficial aquifers (glacial deposits and Pottsville Formation) are exchanged for sodium ions on clay minerals in the shales in the Cuyahoga Group. This exchange process is reflected in a decrease in the median $\mathrm{Ca}: \mathrm{Na}$ equivalent ratio from 10.2 in the waters from the glacial deposits and the Pottsville Formation to 0.15 in the waters from the Cuyahoga Group.

The chemical character of ground waters in the glacial deposits and the Pottsville Formation does not appear to change appreciably across the County. Spatial variations in the chemical character of waters from the Cuyahoga Group appear to be related to well design and stratigraphic proximity to overlying glacial deposits that provide recharge to wells. The highly variable chemical character of waters from the Berea Sandstone generally reflects downward leakage of waters from the Cuyahoga Group in ground-water recharge areas and induced vertical leakage of waters from overlying glacial deposits in wells near ground-water discharge areas. 
On the basis of ground-water-quality analyses from 1978, 1980, and 1986, there do not appear to be any long-term temporal variations in ground-water quality in the various geologic units that are widespread across the County. However, $\mathrm{Br} / \mathrm{Cl}$ mixing diagrams indicate that wells in several localized areas appear to be affected by oilfield brine and the use of sodium chloride and calcium chloride as highway-deicing salts or by storage of these deicing salts at highway garages. Temporal variations from these contaminant sources are reflected in increases or decreases primarily in calcium, sodium, and chloride concentrations as a result of the nature of the source and amount of dilution by recharge.

Generally, waters that are unaffected by oilfield brine and highway-deicing salts conform to USEPA Drinking-Water Regulations. Waters that exceed these regulations generally do not conform to Secondary Drinking-Water Regulations; nonhealth-related criteria (SMCLs).

A regional, steady-state, three-dimensional, finite-difference, ground-water-flow model was constructed, calibrated, and used to determine flow paths and residence times within the various hydrostratigraphic units and to estimate potential water-level declines resulting from forecasted increases in population and concomitant increases in groundwater pumpage. An areally uniform grid was used to encompass the entire County and adjacent areas at a 3,500-ft grid spacing. Model boundaries were designed to coincide with natural hydrologic boundaries within or outside Geauga County. The uppermost model layer represented saturated portions of the glacial deposits and the Pottsville Formation. The Cuyahoga Group was represented by the middle layer of the model, and the Berea Sandstone was represented by the lowermost model layer. Spatial variations in transmissivity in each model layer and recharge to the top layer, as well as pumpage by major ground-water users, were incorporated in the model.

The model was calibrated against assumed steady-state conditions based on water levels measured in January-February 1986 and data from a stream gain/loss study of the Cuyahoga, Chagrin, and Grand Rivers and their tributaries. The simulated steady-state ground-water budget indicates that precipitation accounts for 100 percent of recharge to the ground-water-flow system within the modeled area. Approximately 69 percent of ground-water discharge is to streams and lakes, 29 percent is flow across aquifer boundaries, and 2 percent is pumpage from municipal wells.

Results of the calibrated steady-state-flow model were used in conjunction with a particle-tracking program to determine flow paths and advective residence times within each model layer. Flow paths computed by use of this program are similar to generalized flow directions drawn perpendicular to equipotential lines. Ground water in the glacial deposits and Pottsville Formation discharges along the northern, eastern, and western parts of the County and to the streams within the County. Flow in the Cuyahoga Group is predominantly downward within the interior of the County, whereas flow in the Berea Sandstone is toward the northern, eastern, and western boundaries of the County. 
Ground-water residence times were computed on the basis of advective flow velocities as a means of estimating the duration that a conservative contaminant may reside within the flow system. Depending on the path chosen to represent flow, residence times ranged from 15 to 4,800 years. This range of residence times does not represent all particles of water in the ground-water-flow system.

Simulations of water-level decline were made for several population-growth and distribution scenarios for the years 1985-1995, 1985-2000, and 1985-2005. It was assumed that (1) the ground-water-flow system would attain a new steady-state condition after increased ground-water development, (2) the estimate for the 1985 population within Geauga County represents the population within the County in January-February 1986, (3) all the increased pumpage would be from fully penetrating wells completed in the glacial deposits or the Pottsville Formation, and (4) no water produced from these wells would be returned to the aquifers through septic systems.

Results of the first simulation based on the forecasted 1995 population of Geauga County indicate that up to $4 \mathrm{ft}$ of drawdown could occur locally. An additional $2 \mathrm{ft}$ of drawdown could occur in these areas based on the population forecasted for the year 2000. A total of $8 \mathrm{ft}$ of local drawdown could occur if the forecasted population for the year 2005 is achieved. Because the numerical model is regional in nature and provides a solution that represents every $0.44 \mathrm{mi}^{2}$ with a single ground-water level, actual drawdown near pumped wells could be greater than these simulated average drawdowns. The areas of greatest simulated drawdown are in Chester Township, South Russell Village, the southern part of Russell Township, and Bainbridge Township. On the basis of the estimated distribution of population increase and the concomitant increase in ground-water pumpage, no other areas are expected to experience these relative ground-water-level declines. These predictions are regional in character and should not be used to make sitespecific estimates where more refined interpretations are desired.

\section{REFERENCES CITED}

American Public Health Association, 1980, Standard methods for the examination of water and wastewater (15th ed.): New York, American Public Health Association, Inc., $1134 \mathrm{p}$.

Baker, Jack, 1964, Geology and ground-water resources of Geauga County, Ohio: Unpublished manuscript on file in Columbus, Ohio, office of the U.S. Geological Survey, $108 \mathrm{p}$.

Brodsky, M. H., and Schiemann, D. A., 1976, Evaluation of Pfizer selective enterococcus and KF media for recovery of fecal streptococci from water by membrane filtration: Applied and Environmental Microbiology, v. 31, no. 5, p. 695-699. 
Brown, E., Skougstad, M. W., and Fishman, M. J., 1970, Methods for collection and analysis of water samples for dissolved minerals and gases: U.S. Geological Survey Techniques of Water-Resources Investigations, book 5, chap. A1, $160 \mathrm{p}$.

Davis, S. N., and DeWeist, R. J. M., 1966, Hydrogeology: New York, Wiley, 463 p.

Drever, J. I., 1982, The geochemistry of natural waters: Englewood Cliffs, New Jersey, Prentice-Hall, 388 p.

Eberle, Michael, and McClure, J. A., 1984, Water use in Ohio, 1980: U.S. Geological Survey Water-Resources Investigations Report 84-4024, 34 p.

Fenneman, N. M., 1938, Physiography of eastern United States: New York, McGrawHill, p. 304-319.

Fishman, M. J., and Friedman, L. C., 1985, Methods for determination of inorganic substances in water and fluvial sediments: U.S. Geological Survey Open-File Report 85-495, 709 p.

Gray, J. D., 1981a, Structure on the Berea Sandstone in eastern Ohio, Geauga County: Ohio Geological Survey Open-File Map 132, 1 sheet, scale 1:62,500.

Gray, J. D., 1981b, Structure on the Berea Sandstone in eastern Ohio, Portage County: Ohio Geological Survey Open-File Map 151, 1 sheet, scale 1:62,500.

Greeson, P. E., Ehlke, T. A., Irwin, G. A., Lium, B. W., and Slack, K. V., eds., 1977, Methods for collection and analysis of aquatic biological and microbiological samples: U.S. Geological Survey Techniques of Water-Resources Investigations, book 5, chap. A4, pt. 2, p. 53-62.

Harris Publishing Co., 1985, Harris Ohio industrial directory: Twinsburg, Ohio, p. 196200. Hem, J. D., 1985, Study and interpretation of the chemical characteristics of natural water: U.S. Geological Survey Water-Supply Paper 2254, 263 p.

Jenkins, T. F., 1987, The geology and groundwater resources of Munson Township, Geauga County, Ohio: AGW Consultants, Inc., 117 p.

Knuth, M., Jackson, J. L., and Whittemore, D. O., 1990, An integrated approach to identifying the salinity source contaminating a ground-water supply: Ground Water, v. 28 , no. 3 , p. $202-214$.

McDonald, M. G., and Harbaugh, A. W., 1984, A modular three- dimensional finitedifference ground-water flow model: U.S. Geological Survey Open-File Report 83-875, $528 \mathrm{p}$. 
Nichols, V. E., 1980, Ground-water levels and chemical quality in Geauga County, Ohio, 1978: U.S. Geological Survey Water-Resources Investigations 80-28, 17 p.

Northeast Ohio Area Coordinating Agency, 1983, Population projections for minor civil divisions in the NOACA region: Cleveland, Ohio, $22 \mathrm{p}$.

Ohio Drilling Company, 1971, Ground water potential of northeast Ohio: Massillon, Ohio, $360 \mathrm{p}$.

Pagel, J. E., and Hardy, G. M., 1980, Comparison of selective media for the enumeration and identification of fecal streptococci from natural sources, Canadian Journal of Microbiology, v. 26, p. 1320-1327.

Pettyjohn, W. A., and Henning, R. J., 1979, Preliminary estimate of regional effective ground-water recharge rates in Ohio: The Ohio State University, Water Resources Center, Project Completion Report 552, 323 p.

Pollock, D. W., 1989, Documentation of computer programs to compute and display pathlines using results from the U.S. Geological Survey modular three-dimensional finite-difference ground-water-flow model: U.S. Geological Survey Open-File Report 89-381, 188 p.

Rau, J. L., 1969, Hydrogeology of the Berea and Cussewago sandstones in northeastern Ohio: U.S. Geological Survey Hydrologic Investigations Atlas HA-341, 2 sheets.

Reeder, N. E., and Riemenschneider, V. L., 1973, Soil survey of Ashtabula County, Ohio: U.S. Department of Agriculture, Soil Conservation Service, 114 p.

Risser, M. L., 1983, Top-of-rock map of Portage County, Ohio: Ohio Geological Survey Open-File Map 204, 1 sheet, scale 1:62,500.

Ritchie, A., Bauder, J. R., and Christman, R. L., 1978, Soil survey of Portage County, Ohio: U.S. Department of Agriculture, Soil Conservation Service, 113 p.

Ritchie, A., and Reeder, N. E., 1979, Soil survey of Lake County, Ohio: U.S. Department of Agriculture, Soil Conservation Service, 121 p.

Sedam, A. C., 1973, Hydrogeology of the Pottsville Formation in northeastern Ohio: U.S. Geological Survey Hydrologic Investigations Atlas HA-494, 2 sheets.

Theis, C. V., Brown, R. H., and Meyer, R. R., 1963, Estimating the transmissibility of aquifers from the specific capacity of wells: U.S. Geological Survey Water-Supply Paper 1536-I, p. 331-341. 
Totten, S. M., 1988, Glacial geology of Geauga County, Ohio: Ohio Geological Survey Report of Investigations 140, $1 \mathrm{pl}$.

U.S. Bureau of the Census, 1983, 1980 Census of population: v.1, chap. A, p. 37-8; chap. B, p. 1-11.

U.S. Environmental Protection Agency, 1989a, Final rule, National primary drinking water regulations; Giardia lamlia, viruses, Legionella, and total coliforms, (subparts F and G of part 141): U.S. Federal Register, v. 54, no. 124, June 29, 1989, P. 27 , 486-27,568.

- 1989b, Proposed rule, National primary and secondry drinking water regulations; (sections 141.50, 141.51, 141.61, and 141.62 of part 141 and 143.3 of part 143)

U.S. Federal Register, v. 54, no. 97, May 22, 1989, P. 22, 062-22, 160

_ 1988a, Maximum contaminant levels (subpart B of part 141, National interim primary drinking-water regulations): U.S. Code of Federal Regulations, Title 40, Parts 100 to 149 , revised as of July 1, 1988, p. 530-533.

_ 1988b, Maximum contaminant level goals (subpart F of part 141, National interim primary drinking-water regulations): U.S. Code of Federal Regulations, Title 40, Parts 100 to 149, revised as of July 1, 1988, p. 585-586.

1988c, National revised primary drinking water regulations: Maximum contaminant levels (subpart $\mathrm{G}$ of part 141, National interim primary drinking-water regulations): U.S. Code of Federal Regulations, Title 40, Parts 100 to 149, revised as of July 1, 1988, p. 586-587.

1988d, Secondary maximum contaminant levels (section 143.3 of part 143, National secondary drinking-water regulations): U.S. Code of Federal Regulations, Title 40, Parts 100 to 149, revised as of July 1, 1988, p. 608.

U.S. Geological Survey, Office of Water Data Coordination, 1977, National handbook of recommended methods for water-data acquisition.

Vormelker, J. D., 1981, Top-of-rock map of Geauga County, Ohio: Ohio Geological Survey Open-File Map 117, scale 1:62,500.

Walton, W. C., 1970, Ground water resource evaluation: New York, McGraw-Hill.

White, G. W., 1971, Glacial geology of Trumbull County, Ohio: Ohio Geological Survey Report of Investigations 80, color map with text, 1 sheet, scale 1:62,500. 
White, G. W., 1980, Glacial geology of Lake County, Ohio: Ohio Geological Survey Report of Investigations 117, $20 \mathrm{p}$.

White, G. W., and Totten, S. M., 1979, Glacial geology of Ashtabula County, Ohio: Ohio Geological Survey Report of Investigations 112, 48 p.

Whittemore, D. O., 1984, Geochemical identification of salinity sources, in R.H. French, (ed.), "Salinity in Watercourses and Reservoirs," Ann Arbor Science, Butterworth Publishers, Boston, Massachusetts, p. 505-514.

Whittemore, D. O., 1988, Bromide as a tracer in ground-water studies: Geochemistry and analytical determination: Proceedings, Ground Water Geochemistry Conference, Association of Ground Water Scientists and Engineers, Denver, Colorado, p. 339-359.

Williams, N. L., 1986, An inventory of Ohio soils, Trumbull County: Division of Soil and Water Conservation, Ohio Department of Natural Resources, 69 p.

Williams, N. L., and McCleary, F. E., 1982, Soil survey of Geauga County, Ohio: U.S. Department of Agriculture, Soil Conservation Service, 169 p.

Winslow, J. D., and White, G. W., 1966, Geology and ground-water resources of Portage County, Ohio: U.S. Geological Survey Professional Paper 511, 80 p.

\section{GLOSSARY}

The following are definitions of selected technical terms as they are used in this report; they are not necessarily the only valid definitions for these terms. Terms defined in the glossary are in bold print where first used in the main body of this report.

Advective flow.-The bulk motion of flowing ground water. Advective flow does not account for the spreading of a liquid substance as it moves through a ground-waterflow system.

Anion.-An atom, group of atoms, or molecule that have a negative charge.

Aquifer.-A layer of sediments or rocks that is porous and permeable enough to conduct a useful amount of water. 
Aquifer test.-A field test that involves pumping a well to analyze the change in groundwater levels with time.

Base flow.-The ground-water contribution to streamflow.

Brine.-Water having a total-dissolved-solids concentration greater than 100,000 milligrams per liter.

Buried valley.-A valley from an ancient land surface now filled with glacial deposits.

Cation.-An atom, group of atoms, or molecule that have a positive charge.

Confining unit.-A layer of sediments or rocks that has low permeability and impedes the movement of water into and out of adjacent aquifers.

Contour.-A line joining points of equal value. In this report, it refers to a line of equal altitude.

Darcy's law.-States that the rate of flow through a porous medium is proportional to the loss of hydraulic head, inversely proportional to the length of the flow path, and proportional to hydraulic conductivity.

Effective porosity.-The amount of interconnected pore space in sediments or rocks available to transmit water.

Geohydrology.-Refers to the occurrence, distribution, and movement of ground water.

Hydraulic conductivity.-The volume of water that will flow through a cross-sectional area under a specific gradient during a specific length of time.

Hydraulic head.-The height above a given datum to which a column of ground water will rise.

Hydrograph.-A graph showing water levels or another property of water with respect to time.

Hydrologic boundary. - A boundary of a ground-water-flow system.

Hydrostratigraphic unit.-Sedimentary rocks grouped because they are hydraulically distinct. 
Oxidation-reduction reaction.-A chemical reaction where one element loses an electron and an associated element simultaneously gains an electron.

Potentiometric surface.-A surface defined by the levels to which water will rise in tightly cased wells in an aquifer.

Recharge.-The processes involved in the replenishment of ground water or a quantity of water that replenishes ground water.

Specific capacity.-The yield of a well per unit of drawdown.

Specific conductance.-A measure of the ability of water to conduct an electrical current.

Steady state.-In equilibrium. In terms of ground-water-flow systems, the amount of water leaving the system equals the amount of water entering the system.

Stratigraphic unit.-Sedimentary rocks grouped for description, mapping, and correlation.

Till.-Unsorted and stratified glacial deposit consisting of a heterogeneous mixture of clay, silt, sand, gravel, and boulders varying in size, shape, and composition.

Transmissivity.-The hydraulic conductivity of an aquifer multiplied by the saturated thickness of the aquifer. 
Table 2.--Records of selected wells in Geauga, Lake, Ashtabula, Trumbull, and Portage counties, Ohio

[Aquifer code: 1120TSH, glacial deposits; 324PSVL, Pottsville Formation; 330CyHG, Cuyahoga Group; 330 BERE, Eerea Sandstone.]

\begin{tabular}{|c|c|c|c|c|c|c|}
\hline $\begin{array}{c}\text { Wel } 1 \\
\text { number }\end{array}$ & $\begin{array}{l}\text { Latitude } \\
\text { (degrees) }\end{array}$ & $\begin{array}{l}\text { Longitude } \\
\text { (d̄egrees) }\end{array}$ & $\begin{array}{l}\text { Aquifer } \\
\text { code }\end{array}$ & $\begin{array}{l}\text { Altitude } \\
\text { of land } \\
\text { surface } \\
\text { (feet) }\end{array}$ & $\begin{array}{c}\text { Date } \\
\text { water } \\
\text { leve } 1 \\
\text { measured }\end{array}$ & $\begin{array}{l}\text { Water- } \\
\text { ievel } \\
\text { altitucie } \\
\text { (feet) }\end{array}$ \\
\hline$G E-22$ & 412331 & 0811230 & 324 PSVL & 1,160 & $\begin{array}{l}06-08-78 \\
10-19-78 \\
04-09-80 \\
11-12-80 \\
05-10-85 \\
08-16-85\end{array}$ & $\begin{array}{l}1,145 \\
1,150 \\
1,145 \\
1,145 \\
1,145 \\
1,145\end{array}$ \\
\hline \multirow[t]{2}{*}{$G E-23$} & 412309 & 0812024 & $324 \mathrm{PSVL}$ & 1,150 & $\begin{array}{l}04-26-78 \\
10-19-78 \\
04-08-80 \\
11-06-80 \\
05-10-85\end{array}$ & $\begin{array}{l}1,150 \\
1,145 \\
1,150 \\
1,145 \\
1,145\end{array}$ \\
\hline & & & & & $\begin{array}{l}08-15-85 \\
02-04-86 \\
05-07-86 \\
08-18-86 \\
11-03-86\end{array}$ & $\begin{array}{l}1,145 \\
1,150 \\
1,150 \\
1,145 \\
1,145\end{array}$ \\
\hline \multirow[t]{4}{*}{$\mathrm{GE}-29$} & 412449 & 0812327 & $330 \mathrm{BERE}$ & 955 & $\begin{array}{l}05-03-78 \\
06-03-80 \\
06-16-80 \\
07-02-80 \\
07-31-80\end{array}$ & $\begin{array}{l}915 \\
915 \\
915 \\
915 \\
915\end{array}$ \\
\hline & & & & & $\begin{array}{l}08-13-80 \\
09-17-80 \\
10-01-80 \\
10-19-80 \\
11-05-80\end{array}$ & $\begin{array}{l}915 \\
915 \\
915 \\
910 \\
915\end{array}$ \\
\hline & & & & & $\begin{array}{l}06-25-81 \\
05-12-85 \\
08-28-85 \\
02-04-86 \\
05-07-86\end{array}$ & $\begin{array}{l}915 \\
915 \\
915 \\
915 \\
915\end{array}$ \\
\hline & & & & & $\begin{array}{l}08-18-86 \\
11-03-86\end{array}$ & $\begin{array}{l}915 \\
915\end{array}$ \\
\hline
\end{tabular}


Table 2.--Records of selected wells in Geauga, Lake, Ashtabula, Trumbul1, and Portage Counties, Ohio--Continued

\begin{tabular}{|c|c|c|c|c|c|c|}
\hline $\begin{array}{c}\text { Wel } 1 \\
\text { number } r\end{array}$ & $\begin{array}{l}\text { Latitude } \\
\text { (degrees) }\end{array}$ & $\begin{array}{l}\text { Longitude } \\
\text { (degrees) }\end{array}$ & $\begin{array}{l}\text { Aqui fer } \\
\text { code }\end{array}$ & $\begin{array}{l}\text { Altitude } \\
\text { of land } \\
\text { surface } \\
\text { (feet) }\end{array}$ & $\begin{array}{c}\text { Date } \\
\text { water } \\
\text { level. } \\
\text { measurea }\end{array}$ & $\begin{array}{l}\text { water- } \\
\text { level } \\
\text { altitude } \\
\text { (feet) }\end{array}$ \\
\hline \multirow[t]{2}{*}{$\mathrm{GE}-31$} & 412655 & 0812056 & 324 PSVL & 1,110 & $\begin{array}{l}05-03-78 \\
10-19-78 \\
04-08-80 \\
11-05-80 \\
05-10-85\end{array}$ & $\begin{array}{l}1,110 \\
1,110 \\
1,110 \\
1,110 \\
1,110\end{array}$ \\
\hline & & & & & $\begin{array}{l}08-13-85 \\
01-31-86 \\
05-08-86 \\
08-20-86 \\
11-06-86\end{array}$ & $\begin{array}{l}1,110 \\
1,110 \\
1,110 \\
1,110 \\
1,110\end{array}$ \\
\hline \multirow[t]{2}{*}{$\mathrm{GE}-36$} & 412439 & 0811830 & $324 \mathrm{PSVL}$ & 1.260 & $\begin{array}{l}05-04-78 \\
10-19-78 \\
04-09-80 \\
11-06-80 \\
05-10-85\end{array}$ & $\begin{array}{l}1,195 \\
1,195 \\
1,195 \\
1,195 \\
1,190\end{array}$ \\
\hline & & & & & $\begin{array}{l}08-27-85 \\
02-04-86 \\
05-07-86 \\
08-18-86 \\
11-03-86\end{array}$ & $\begin{array}{l}1,190 \\
1,195 \\
1,195 \\
1,195 \\
1,195\end{array}$ \\
\hline $\mathrm{GE}-39$ & 412444 & 0812021 & 324 PSVL & 1,200 & $\begin{array}{l}04-25-86 \\
05-09-86 \\
08-18-86 \\
11-03-86\end{array}$ & $\begin{array}{l}1,160 \\
1,150 \\
1,160 \\
1,160\end{array}$ \\
\hline \multirow[t]{2}{*}{$\mathrm{GE}-42$} & 412901 & 0810453 & $1120 \mathrm{TSH}$ & 1,105 & $\begin{array}{l}05-09-78 \\
10-19-78 \\
04-10-80 \\
11-12-80 \\
05-09-85\end{array}$ & $\begin{array}{l}1,065 \\
1,065 \\
1,065 \\
1,065 \\
1,060\end{array}$ \\
\hline & & & & & $\begin{array}{l}08-08-85 \\
02-05-86 \\
05-08-86 \\
08-19-86 \\
11-04-86\end{array}$ & $\begin{array}{l}1,050 \\
1,055 \\
1,070 \\
1,055 \\
1,065\end{array}$ \\
\hline
\end{tabular}


Table 2.--Records of selected wells in Geauga, Lake, Ashtabula, Trumbull, and Portage Counties, Ohio--Continued

\begin{tabular}{|c|c|c|c|c|c|c|}
\hline $\begin{array}{l}\text { We11 } \\
\text { number }\end{array}$ & $\begin{array}{l}\text { Latitude } \\
\text { (degrees) }\end{array}$ & $\begin{array}{l}\text { Longitude } \\
\text { (d̄egrees) }\end{array}$ & $\begin{array}{l}\text { Aqui fer } \\
\text { code }\end{array}$ & $\begin{array}{l}\text { Altitude } \\
\text { of land } \\
\text { surface } \\
\text { (feet) }\end{array}$ & $\begin{array}{c}\text { Date } \\
\text { water } \\
\text { level } \\
\text { measured }\end{array}$ & $\begin{array}{l}\text { Water- } \\
\text { level } \\
\text { altitude } \\
\text { (feet) }\end{array}$ \\
\hline \multirow[t]{5}{*}{$\mathrm{GE}-45$} & 414026 & 0810244 & 324 PSVL & 1,300 & $\begin{array}{l}05-10-78 \\
10-19-78 \\
01-09-80 \\
01-31-80 \\
02-14-80\end{array}$ & $\begin{array}{l}1,260 \\
1,260 \\
1,260 \\
1,260 \\
1,260\end{array}$ \\
\hline & & & & & $\begin{array}{l}03-03-80 \\
03-18-80 \\
03-31-80 \\
04-11-80 \\
04-30-80\end{array}$ & $\begin{array}{l}1.260 \\
1.260 \\
1.260 \\
1.260 \\
1.260\end{array}$ \\
\hline & & & & & $\begin{array}{l}05-15-80 \\
06-03-80 \\
06-16-80 \\
07-02-80 \\
07-31-80\end{array}$ & $\begin{array}{l}1,260 \\
1,260 \\
1,260 \\
1,260 \\
1,260\end{array}$ \\
\hline & & & & & $\begin{array}{l}08-13-80 \\
09-17-80 \\
10-01-80 \\
11-13-80 \\
06-25-81\end{array}$ & $\begin{array}{l}1,260 \\
1,260 \\
1,260 \\
1,255 \\
1,260\end{array}$ \\
\hline & & & & & $\begin{array}{l}08-05-85 \\
02-11-86 \\
05-08-86\end{array}$ & $\begin{array}{l}1,255 \\
1,260 \\
1,260\end{array}$ \\
\hline \multirow[t]{2}{*}{$G E-48$} & 413202 & 0810157 & $330 \mathrm{CYHG}$ & 1,090 & $\begin{array}{l}05-10-78 \\
10-19-78 \\
01-09-80 \\
01-31-80 \\
02-14-80\end{array}$ & $\begin{array}{l}1,085 \\
1,085 \\
1,085 \\
1,085 \\
1,085\end{array}$ \\
\hline & & & & & $\begin{array}{l}03-03-80 \\
03-18-80 \\
03-31-80 \\
04-10-80 \\
04-30-80\end{array}$ & $\begin{array}{l}1,085 \\
1,085 \\
1,085 \\
1,085 \\
1,085\end{array}$ \\
\hline
\end{tabular}


Table 2.--Fecorảs of selected wells in Geauga, Lake, Ashtabula, Trumbul 1, and Portage Counties, Ohio--Continued

\begin{tabular}{|c|c|c|c|c|c|c|}
\hline $\begin{array}{c}\text { Well } \\
\text { number }\end{array}$ & $\begin{array}{l}\text { Latitude } \\
\text { (degrees) }\end{array}$ & $\begin{array}{l}\text { Longitudie } \\
\text { (ojegrees) }\end{array}$ & $\begin{array}{l}\text { Aqui fer } \\
\text { code }\end{array}$ & $\begin{array}{l}\text { Altitude } \\
\text { of land } \\
\text { surface } \\
\text { (feet) }\end{array}$ & $\begin{array}{c}\text { Date } \\
\text { water } \\
\text { level } \\
\text { measurea }\end{array}$ & $\begin{array}{l}\text { Water- } \\
\text { level } \\
\text { a1titucie } \\
\text { (feet) }\end{array}$ \\
\hline \multirow[t]{3}{*}{$\mathrm{GE}-48-$} & Continueả & & & & $\begin{array}{l}05-15-80 \\
06-03-80 \\
06-16-80 \\
07-02-80 \\
07-31-80\end{array}$ & $\begin{array}{l}1,085 \\
1,085 \\
1,085 \\
1,085 \\
1,085\end{array}$ \\
\hline & & & & & $\begin{array}{l}08-13-80 \\
09-17-80 \\
10-01-80 \\
11-13-80 \\
06-25-81\end{array}$ & $\begin{array}{l}1.085 \\
1.085 \\
1.085 \\
1.085 \\
1.085\end{array}$ \\
\hline & & & & & $\begin{array}{l}08-08-85 \\
04-16-86\end{array}$ & $\begin{array}{l}1,085 \\
1,085\end{array}$ \\
\hline \multirow[t]{2}{*}{$G E-53$} & 413346 & 0811223 & $1120 \mathrm{TSH}$ & 1,150 & $\begin{array}{l}05-11-78 \\
10-19-78 \\
11-13-80 \\
05-11-85 \\
08-06-85\end{array}$ & $\begin{array}{l}1,105 \\
1,105 \\
1,120 \\
1,120 \\
1,125\end{array}$ \\
\hline & & & & & $\begin{array}{l}01-30-86 \\
05-09-86 \\
11-06-86\end{array}$ & $\begin{array}{l}1,115 \\
1,120 \\
1,125\end{array}$ \\
\hline$G E-55$ & 413258 & 0811009 & $330 \mathrm{CYHG}$ & 1,145 & $\begin{array}{l}05-09-85 \\
08-08-85 \\
02-07-86 \\
04-17-86\end{array}$ & $\begin{array}{l}1,145 \\
1,145 \\
\text { Flowing } \\
\text { Flowing }\end{array}$ \\
\hline \multirow[t]{2}{*}{$G E-60$} & 412051 & 0811657 & $324 \mathrm{PSVI}$ & 1,200 & $\begin{array}{l}05-24-78 \\
10-19-78 \\
04-09-80 \\
11-06-80 \\
05-10-85\end{array}$ & $\begin{array}{l}1,140 \\
1,140 \\
1,140 \\
1,140 \\
1,140\end{array}$ \\
\hline & & & & & $\begin{array}{l}08-16-85 \\
02-04-86 \\
04-29-86 \\
08-18-86 \\
11-03-86\end{array}$ & $\begin{array}{l}1,140 \\
1,140 \\
1,140 \\
1,140 \\
1,140\end{array}$ \\
\hline
\end{tabular}


Table 2.--Records of selected wells in Geauga, Lake, Ashtabula, Trumbull, and Portage Counties, Ohio--Continued

\begin{tabular}{|c|c|c|c|c|c|c|}
\hline $\begin{array}{l}\text { Wel1 } \\
\text { number }\end{array}$ & $\begin{array}{l}\text { Latitude } \\
\text { (äegrees) }\end{array}$ & $\begin{array}{r}\text { Longitude } \\
\text { (degrees) }\end{array}$ & $\begin{array}{l}\text { Aquifer } \\
\text { code }\end{array}$ & $\begin{array}{l}\text { Altitude } \\
\text { of land } \\
\text { surface } \\
\text { (feet) }\end{array}$ & $\begin{array}{c}\text { Date } \\
\text { water } \\
\text { level } \\
\text { measured }\end{array}$ & $\begin{array}{l}\text { Water- } \\
\text { level } \\
\text { altitude } \\
\text { (feet) }\end{array}$ \\
\hline \multirow[t]{2}{*}{$G E-64$} & 112749 & 0811452 & 324 PSVL & 1,235 & $\begin{array}{l}06-14-78 \\
11-06-80 \\
05-11-85 \\
08-14-85 \\
01-30-86\end{array}$ & $\begin{array}{l}1,210 \\
1,210 \\
1,205 \\
1,205 \\
1,205\end{array}$ \\
\hline & & & & & $\begin{array}{l}05-08-86 \\
08-20-86 \\
11-06-86\end{array}$ & $\begin{array}{l}1,205 \\
1,205 \\
1,205\end{array}$ \\
\hline $\mathrm{GE}-67$ & 412522 & 0810928 & $330 \mathrm{CYHG}$ & 1,100 & $\begin{array}{l}06-08-78 \\
10-19-78 \\
04-09-80 \\
11-06-80 \\
08-09-85 \\
04-24-86\end{array}$ & $\begin{array}{l}1,100 \\
1,095 \\
1,100 \\
1,100 \\
1,095 \\
1,095\end{array}$ \\
\hline \multirow[t]{4}{*}{$G E-68$} & 412949 & 0811046 & 324 PSVL & 1,205 & $\begin{array}{l}06-14-78 \\
10-19-78 \\
01-08-80 \\
01-31-80 \\
02-14-80\end{array}$ & $\begin{array}{l}1,185 \\
1,185 \\
1,190 \\
1,185 \\
1,185\end{array}$ \\
\hline & & & & & $\begin{array}{l}03-03-80 \\
03-18-80 \\
03-31-80 \\
04-10-80 \\
04-30-80 \\
05-15-80 \\
06-03-80 \\
06-16-80 \\
07-02-80 \\
07-31-80\end{array}$ & $\begin{array}{l}1,185 \\
1,185 \\
1,190 \\
1,190 \\
1,190 \\
1,185 \\
1,185 \\
1,185 \\
1,185 \\
1,185\end{array}$ \\
\hline & & & & & $\begin{array}{l}08-13-80 \\
09-17-80 \\
10-01-80 \\
11-13-80 \\
06-25-81\end{array}$ & $\begin{array}{l}1,185 \\
1,185 \\
1,185 \\
1,185 \\
1,185\end{array}$ \\
\hline & & & & & $\begin{array}{l}05-12-85 \\
08-15-85 \\
02-06-86 \\
04-24-86\end{array}$ & $\begin{array}{l}1,185 \\
1,185 \\
1,190 \\
1,185\end{array}$ \\
\hline
\end{tabular}


Table 2.--Records of selected wells in Geauga, Lake, Ashtabula, Trumbull, and Portage Counties, Ohio--Continued

\begin{tabular}{|c|c|c|c|c|c|c|}
\hline $\begin{array}{l}\text { Well } 1 \\
\text { number }\end{array}$ & $\begin{array}{l}\text { Latitude } \\
\text { (degrees) }\end{array}$ & $\begin{array}{l}\text { Longitude } \\
\text { (degrees) }\end{array}$ & $\begin{array}{l}\text { Aqui fer } \\
\text { code }\end{array}$ & $\begin{array}{l}\text { Altitude } \\
\text { of land } \\
\text { surface } \\
\text { (feet) }\end{array}$ & $\begin{array}{c}\text { Date } \\
\text { water } \\
\text { level } \\
\text { measured }\end{array}$ & $\begin{array}{l}\text { Water- } \\
\text { level } \\
\text { altitude } \\
\text { (feet) }\end{array}$ \\
\hline \multicolumn{2}{|c|}{ GE-68--Continued } & & & & $\begin{array}{l}08-19-86 \\
11-04-86\end{array}$ & $\begin{array}{l}1,185 \\
1,185\end{array}$ \\
\hline \multirow[t]{2}{*}{$G E-69$} & 413151 & 0811258 & 324 PSVL & 1,260 & $\begin{array}{l}06-15-78 \\
10-19-78 \\
04-10-80 \\
01-13-80 \\
05-11-85\end{array}$ & $\begin{array}{l}1,240 \\
1,240 \\
1,240 \\
1,235 \\
1,235\end{array}$ \\
\hline & & & & & $\begin{array}{l}08-08-85 \\
01-30-86 \\
04-22-86 \\
08-21-86 \\
11-06-86\end{array}$ & $\begin{array}{l}1,230 \\
1,230 \\
1,230 \\
1,235 \\
1,230\end{array}$ \\
\hline $\mathrm{GE}-70$ & 413201 & 0811109 & 330 CYHG & 1,150 & $\begin{array}{l}05-12-85 \\
08-08-85 \\
08-21-86 \\
11-04-86\end{array}$ & $\begin{array}{l}1,150 \\
1,150 \\
1,150 \\
1,150\end{array}$ \\
\hline \multirow[t]{2}{*}{$\mathrm{GE}-72$} & 413433 & 0810755 & 324 P SVL & 1,220 & $\begin{array}{l}06-15-78 \\
10-19-78 \\
04-11-80 \\
11-14-80 \\
05-09-85\end{array}$ & $\begin{array}{l}1,210 \\
1,205 \\
1,210 \\
1,205 \\
1,205\end{array}$ \\
\hline & & & & & $\begin{array}{l}08-06-85 \\
02-07-86 \\
05-08-86 \\
08-21-86 \\
11-05-86\end{array}$ & $\begin{array}{l}1,205 \\
1,210 \\
1,205 \\
1,205 \\
1,205\end{array}$ \\
\hline
\end{tabular}


Table 2.--Records of selected wells in Geauga, Lake, Ashtabula, Trumbul1, and Portage Counties, Ohio--Continued

\begin{tabular}{|c|c|c|c|c|c|c|}
\hline $\begin{array}{c}\text { Wel } 1 \\
\text { number }\end{array}$ & $\begin{array}{l}\text { Latitude } \\
\text { (diegrees) }\end{array}$ & $\begin{array}{l}\text { Longitude } \\
\text { (degrees) }\end{array}$ & $\begin{array}{l}\text { Aquifer } \\
\text { coaje }\end{array}$ & $\begin{array}{l}\text { Altitude } \\
\text { of land } \\
\text { surface } \\
\text { (feet) }\end{array}$ & $\begin{array}{c}\text { Date } \\
\text { water } \\
\text { level } \\
\text { measured }\end{array}$ & $\begin{array}{l}\text { Water- } \\
\text { level } \\
\text { altitude } \\
\text { (feet) }\end{array}$ \\
\hline$G E-73$ & 413629 & 0810828 & 324 PSVL & 1,300 & $\begin{array}{l}06-15-78 \\
10-19-78 \\
04-11-80 \\
11-13-80 \\
06-25-81 \\
08-06-85 \\
04-15-86\end{array}$ & $\begin{array}{l}1,255 \\
1,255 \\
1,255 \\
1,255 \\
1,255 \\
1,255 \\
1,250\end{array}$ \\
\hline \multirow[t]{5}{*}{$G E-76$} & 413138 & 0811520 & 112OTSH & 1,170 & $\begin{array}{l}06-15-78 \\
10-19-78 \\
01-31-80 \\
02-14-80 \\
03-03-80\end{array}$ & $\begin{array}{l}1,150 \\
1,150 \\
1,145 \\
1,150 \\
1,150\end{array}$ \\
\hline & & & & & $\begin{array}{l}03-18-80 \\
03-31-80 \\
04-10-80 \\
04-30-80 \\
05-15-80\end{array}$ & $\begin{array}{l}1,150 \\
1,150 \\
1,150 \\
1,150 \\
1,150\end{array}$ \\
\hline & & & & & $\begin{array}{l}06-03-80 \\
06-16-80 \\
07-02-80 \\
07-31-80 \\
08-13-80\end{array}$ & $\begin{array}{l}1,150 \\
1,150 \\
1,150 \\
1,150 \\
1,150\end{array}$ \\
\hline & & & & & $\begin{array}{l}09-17-80 \\
10-01-80 \\
11-06-80 \\
06-25-81 \\
05-12-85\end{array}$ & $\begin{array}{l}1,145 \\
1,145 \\
1,150 \\
1,150 \\
1,145\end{array}$ \\
\hline & & & & & $\begin{array}{l}08-07-85 \\
01-30-86 \\
05-09-86 \\
08-21-86 \\
11-06-86\end{array}$ & $\begin{array}{l}1,145 \\
1,145 \\
1,145 \\
1,145 \\
1,145\end{array}$ \\
\hline
\end{tabular}


Table 2.--Records of selected ivells in Geauga, Iake, Ashtabula, Trumbull, and Portage Counties, Ohio--Continued

\begin{tabular}{|c|c|c|c|c|c|c|}
\hline $\begin{array}{l}\text { Wel1 } \\
\text { number }\end{array}$ & $\begin{array}{l}\text { Latitude } \\
\text { (degrees) }\end{array}$ & $\begin{array}{l}\text { Longitude } \\
\text { (d̃egrees) }\end{array}$ & $\begin{array}{l}\text { Aquifer } \\
\text { cocie }\end{array}$ & $\begin{array}{l}\text { Altitucie } \\
\text { of land } \\
\text { surface } \\
\text { (feet) }\end{array}$ & $\begin{array}{c}\text { Date } \\
\text { wáter } \\
\text { level } \\
\text { measureà }\end{array}$ & $\begin{array}{l}\text { Water- } \\
\text { level } \\
\text { altituce } \\
\text { (feet) }\end{array}$ \\
\hline \multirow[t]{2}{*}{$\mathrm{GE}-77$} & 413028 & 0812210 & $330 \mathrm{CYHG}$ & 1,140 & $\begin{array}{l}06-15-78 \\
10-19-78 \\
01-08-80 \\
11-05-80 \\
05-11-85\end{array}$ & $\begin{array}{l}1,100 \\
1,100 \\
1,100 \\
1,105 \\
1,100\end{array}$ \\
\hline & & & & & $\begin{array}{l}08-07-85 \\
01-29-86 \\
04-22-86\end{array}$ & $\begin{array}{l}1,100 \\
1,100 \\
1,105\end{array}$ \\
\hline \multirow[t]{2}{*}{$G E-83$} & 412627 & 0810705 & 324 PSVL & 1,220 & $\begin{array}{l}05-14-78 \\
10-19-78 \\
04-10-80 \\
11-12-80 \\
05-12-85\end{array}$ & $\begin{array}{l}1,190 \\
1,190 \\
1,190 \\
1,185 \\
1,190\end{array}$ \\
\hline & & & & & $\begin{array}{l}08-14-85 \\
02-06-86 \\
05-08-86 \\
08-19-86 \\
11-04-86\end{array}$ & $\begin{array}{l}1,190 \\
1,190 \\
1,190 \\
1,190 \\
1,190\end{array}$ \\
\hline $\mathrm{GE}-89$ & 412749 & 0811715 & 324 PSVL & 1,270 & $\begin{array}{l}05-11-85 \\
08-14-85 \\
01-30-86 \\
04-22-86 \\
08-20-86 \\
11-06-86\end{array}$ & $\begin{array}{l}1,185 \\
1,185 \\
1,185 \\
1,185 \\
1,185 \\
1,185\end{array}$ \\
\hline \multirow[t]{2}{*}{$\mathrm{GE}-91$} & 412748 & 0811439 & 112OTSH & 1,250 & $\begin{array}{l}10-19-78 \\
04-10-80 \\
11-06-80 \\
05-11-85 \\
08-14-85\end{array}$ & $\begin{array}{l}1,210 \\
1,210 \\
1,210 \\
1,205 \\
1,205\end{array}$ \\
\hline & & & & & $\begin{array}{l}01-30-86 \\
05-08-86 \\
08-20-86 \\
11-06-86\end{array}$ & $\begin{array}{l}1,205 \\
1,205 \\
1.205 \\
1.205\end{array}$ \\
\hline
\end{tabular}


Table 2.--Recoras of selected wells in Geauga, Lake, Ashtabula, Trumbul1, and Portage Counties, Ohio--Continued

\begin{tabular}{|c|c|c|c|c|c|c|}
\hline $\begin{array}{c}\text { well } 1 \\
\text { number }\end{array}$ & $\begin{array}{l}\text { Iatitucie } \\
\text { (ciegrees) }\end{array}$ & $\begin{array}{l}\text { Longitude } \\
\text { (degrees) }\end{array}$ & $\begin{array}{l}\text { Aquifer } \\
\text { code }\end{array}$ & $\begin{array}{l}\text { Altitude } \\
\text { of land } \\
\text { surface } \\
\text { (feet) }\end{array}$ & $\begin{array}{c}\text { Date } \\
\text { water } \\
\text { level } \\
\text { measur ed }\end{array}$ & $\begin{array}{l}\text { Water- } \\
\text { level } \\
\text { altitude } \\
\text { (feet) }\end{array}$ \\
\hline$G E-92$ & 412713 & 0811232 & $1120 T S H$ & 1,170 & $\begin{array}{l}10-20-78 \\
04-10-80 \\
11-06-80 \\
05-12-85 \\
08-14-85 \\
04-24-86\end{array}$ & $\begin{array}{l}1,135 \\
1,135 \\
1,135 \\
1,135 \\
1,135 \\
1,135\end{array}$ \\
\hline GE-101 & 413757 & 0811223 & $1120 \mathrm{TSH}$ & 990 & $\begin{array}{l}05-07-80 \\
08-06-85 \\
01-29-86 \\
04-14-86 \\
08-21-86 \\
11-05-86\end{array}$ & $\begin{array}{l}970 \\
965 \\
970 \\
965 \\
965 \\
965\end{array}$ \\
\hline $\mathrm{GE}-102$ & 413450 & 0811730 & $330 \mathrm{BERE}$ & 1,025 & $\begin{array}{l}05-07-80 \\
08-27-85 \\
04-15-86 \\
08-21-86\end{array}$ & $\begin{array}{l}975 \\
985 \\
975 \\
985\end{array}$ \\
\hline \multirow[t]{2}{*}{$G E-103$} & 413755 & 0811012 & $330 \mathrm{BERE}$ & 1,160 & $\begin{array}{l}05-07-80 \\
11-14-80 \\
08-06-85 \\
02-07-86 \\
05-01-86\end{array}$ & $\begin{array}{l}1,080 \\
1,080 \\
1,080 \\
1,075 \\
1,070\end{array}$ \\
\hline & & & & & $\begin{array}{l}08-21-86 \\
11-05-86\end{array}$ & $\begin{array}{l}1,070 \\
1,070\end{array}$ \\
\hline \multirow[t]{2}{*}{$G E-104$} & 413606 & 0811021 & $330 \mathrm{BERE}$ & 1,215 & $\begin{array}{l}05-07-80 \\
11-14-80 \\
08-06-85 \\
02-07-86 \\
05-01-86\end{array}$ & $\begin{array}{l}1,125 \\
1,125 \\
1,125 \\
1,120 \\
1,120\end{array}$ \\
\hline & & & & & $\begin{array}{l}08-21-86 \\
11-05-86\end{array}$ & $\begin{array}{l}1,120 \\
1,120\end{array}$ \\
\hline
\end{tabular}


Table 2.--Records of selected wells in Geauga, Lake, Ashtabula, Trumbul1, and Portage Counties, Ohio--Continued

\begin{tabular}{|c|c|c|c|c|c|c|}
\hline $\begin{array}{c}\text { Well } \\
\text { number }\end{array}$ & $\begin{array}{l}\text { Latitude } \\
\text { (degrees) }\end{array}$ & $\begin{array}{l}\text { Longitude } \\
\text { (degrees) }\end{array}$ & $\begin{array}{l}\text { Aqui fer } \\
\text { code }\end{array}$ & $\begin{array}{l}\text { Altitude } \\
\text { of land } \\
\text { surface } \\
\text { (feet) }\end{array}$ & $\begin{array}{c}\text { Date } \\
\text { water } \\
\text { level } \\
\text { measureà }\end{array}$ & $\begin{array}{l}\text { Water- } \\
\text { level. } \\
\text { altitucie } \\
\text { (feet) }\end{array}$ \\
\hline \multirow[t]{2}{*}{$\mathrm{GE}-105$} & 413544 & 0810605 & $330 \mathrm{CYHG}$ & 1,220 & $\begin{array}{l}05-07-80 \\
11-14-80 \\
08-06-85 \\
02-07-86 \\
04-15-86\end{array}$ & $\begin{array}{l}1,185 \\
1,180 \\
1,175 \\
1,195 \\
1,195\end{array}$ \\
\hline & & & & & $\begin{array}{l}08-21-86 \\
11-05-86\end{array}$ & $\begin{array}{l}1,195 \\
1,190\end{array}$ \\
\hline \multirow[t]{2}{*}{$\mathrm{GE}-106$} & 413456 & 0810416 & $330 \mathrm{CYHG}$ & 1.255 & $\begin{array}{l}05-07-80 \\
11-13-80 \\
08-05-85 \\
02-10-86 \\
05-08-86\end{array}$ & $\begin{array}{l}1.225 \\
1.225 \\
1.225 \\
1,220 \\
1.220\end{array}$ \\
\hline & & & & & $\begin{array}{l}08-19-86 \\
11-05-86\end{array}$ & $\begin{array}{l}1.220 \\
1.220\end{array}$ \\
\hline GE-107 & 413249 & 0811732 & 112OTSH & 1,045 & $\begin{array}{l}05-08-80 \\
11-13-80 \\
08-07-85 \\
02-13-85 \\
05-09-86 \\
08-21-86 \\
11-06-86\end{array}$ & $\begin{array}{l}985 \\
985 \\
985 \\
980 \\
985 \\
985 \\
985\end{array}$ \\
\hline \multirow[t]{2}{*}{$\mathrm{GE}-108$} & 413106 & 0811719 & 112OTSH & 1,120 & $\begin{array}{l}05-08-80 \\
1.1-06-80 \\
08-07-85 \\
01-30-86 \\
05-01-86\end{array}$ & $\begin{array}{l}1,070 \\
1,070 \\
1,070 \\
1,070 \\
1,070\end{array}$ \\
\hline & & & & & $\begin{array}{l}08-21-86 \\
11-06-86\end{array}$ & $\begin{array}{l}1,070 \\
1,070\end{array}$ \\
\hline \multirow[t]{2}{*}{ GE-109 } & 413002 & 0811302 & 324 PSVL & 1,280 & $\begin{array}{l}05-08-80 \\
11-07-80 \\
08-07-85 \\
01-30-86 \\
04-22-86\end{array}$ & $\begin{array}{l}1,205 \\
1,205 \\
1,205 \\
1,205 \\
1,205\end{array}$ \\
\hline & & & & & $\begin{array}{l}08-21-86 \\
11-06-86\end{array}$ & $\begin{array}{l}1.205 \\
1.205\end{array}$ \\
\hline
\end{tabular}


Table 2.--Records of selected wells in Geauga, Lake, Ashtabula, Trumbull, and Portage Counties, Ohio--Continued

\begin{tabular}{|c|c|c|c|c|c|c|}
\hline $\begin{array}{c}\text { Well } 1 \\
\text { number }\end{array}$ & $\begin{array}{l}\text { Latitude } \\
\text { (degrees) }\end{array}$ & $\begin{array}{l}\text { Longitude } \\
\text { (ảegrees) }\end{array}$ & $\begin{array}{l}\text { Aqui fer } \\
\text { coảe }\end{array}$ & $\begin{array}{l}\text { Altitude } \\
\text { of land } \\
\text { surface } \\
\text { (feet) }\end{array}$ & $\begin{array}{c}\text { Date } \\
\text { water } \\
\text { level } \\
\text { measur eå }\end{array}$ & $\begin{array}{l}\text { Water- } \\
\text { level } \\
\text { altitude } \\
\text { (feet) }\end{array}$ \\
\hline $\mathrm{GE}-110$ & 413049 & 0810839 & 324 PSVL & 1,280 & $\begin{array}{l}05-08-80 \\
11-13-80 \\
08-08-85\end{array}$ & $\begin{array}{l}1,245 \\
1,240 \\
1,240\end{array}$ \\
\hline \multirow[t]{2}{*}{$\mathrm{GE}-111$} & 413346 & 0810632 & 324 PSVL & 1,270 & $\begin{array}{l}05-08-80 \\
11-14-80 \\
08-08-85 \\
02-06-86 \\
04-17-86\end{array}$ & $\begin{array}{l}1,245 \\
1,245 \\
1,245 \\
1,240 \\
1,240\end{array}$ \\
\hline & & & & & $\begin{array}{l}08-21-86 \\
11-04-86\end{array}$ & $\begin{array}{l}1,245 \\
1,240\end{array}$ \\
\hline \multirow[t]{2}{*}{$\mathrm{GE}-112$} & 413207 & 0810444 & 324 PSVL & 1.265 & $\begin{array}{l}05-05-80 \\
05-08-80 \\
11-14-80 \\
08-08-85 \\
02-06-86\end{array}$ & $\begin{array}{l}1,220 \\
1,220 \\
1,220 \\
1,220 \\
1,220\end{array}$ \\
\hline & & & & & $\begin{array}{l}04-17-86 \\
08-19-86 \\
11-04-86\end{array}$ & $\begin{array}{l}1,220 \\
1,220 \\
1,220\end{array}$ \\
\hline \multirow[t]{2}{*}{$\mathrm{GE}-113$} & 413633 & 0810518 & 330 CYHG & 1,250 & $\begin{array}{l}05-08-80 \\
11-13-80 \\
08-06-85 \\
02-10-86 \\
05-08-86\end{array}$ & $\begin{array}{l}1,230 \\
1,230 \\
1,230 \\
1,225 \\
1,225\end{array}$ \\
\hline & & & & & $\begin{array}{l}08-19-86 \\
11-05-86 \\
11-19-86\end{array}$ & $\begin{array}{l}1,225 \\
1,220 \\
1,225\end{array}$ \\
\hline \multirow[t]{2}{*}{$\mathrm{GE}-114$} & 412900 & 0810710 & 324 PSVL & 1,265 & $\begin{array}{l}05-09-80 \\
11-12-80 \\
08-08-85 \\
02-06-86 \\
05-08-86\end{array}$ & $\begin{array}{l}1,225 \\
1,225 \\
1,215 \\
1,220 \\
1,220\end{array}$ \\
\hline & & & & & $\begin{array}{l}08-18-86 \\
11-04-86\end{array}$ & $\begin{array}{l}1,220 \\
1,220\end{array}$ \\
\hline
\end{tabular}


Table 2.--Records of selected wells in Geauga, Lake, Ashtabula, Trumbull, and Portage Counties, Ohio--Continued

\begin{tabular}{|c|c|c|c|c|c|c|}
\hline $\begin{array}{l}\text { Wel } 1 \\
\text { number }\end{array}$ & $\begin{array}{l}\text { Latitude } \\
\text { (degrees) }\end{array}$ & $\begin{array}{l}\text { Longitude } \\
\text { (degrees) }\end{array}$ & $\begin{array}{l}\text { Aqui fer } \\
\text { code }\end{array}$ & $\begin{array}{l}\text { Altitude } \\
\text { of land } \\
\text { surface } \\
\text { (feet) }\end{array}$ & $\begin{array}{c}\text { Date } \\
\text { water } \\
\text { level } \\
\text { measured }\end{array}$ & $\begin{array}{l}\text { Water- } \\
\text { level } \\
\text { altitude } \\
\text { (feet) }\end{array}$ \\
\hline \multirow[t]{2}{*}{ GE -115} & 412737 & 0810633 & 324 PSVL & 1,170 & $\begin{array}{l}05-09-80 \\
11-12-80 \\
08-14-85 \\
02-06-86 \\
04-24-86\end{array}$ & $\begin{array}{l}1,145 \\
1,145 \\
1,145 \\
1,145 \\
1,145\end{array}$ \\
\hline & & & & & $\begin{array}{l}08-19-86 \\
11-04-86\end{array}$ & $\begin{array}{l}1,145 \\
1,145\end{array}$ \\
\hline \multirow[t]{2}{*}{$\mathrm{GE}-116$} & 412926 & 0811443 & $1120 \mathrm{TSH}$ & 1.180 & $\begin{array}{l}05-09-80 \\
11-06-80 \\
08-14-85 \\
01-30-86 \\
04-30-86\end{array}$ & $\begin{array}{l}1,145 \\
1,145 \\
1,145 \\
1,145 \\
1,145\end{array}$ \\
\hline & & & & & $\begin{array}{l}08-20-86 \\
11-06-86\end{array}$ & $\begin{array}{l}1,145 \\
1,145\end{array}$ \\
\hline GE-117 & 412600 & 0811448 & 324 PSVL & 1,205 & $\begin{array}{l}05-09-80 \\
11-06-80 \\
08-14-85 \\
02-13-86 \\
05-08-86 \\
08-20-86\end{array}$ & $\begin{array}{l}1,190 \\
1,190 \\
1,185 \\
1,190 \\
1,190 \\
1,185\end{array}$ \\
\hline \multirow[t]{2}{*}{$\mathrm{GE}-118$} & 412915 & 0810459 & 33 OCYHG & 1,125 & $\begin{array}{l}06-25-80 \\
08-13-80 \\
09-17-80 \\
10-01-80 \\
11-12-80\end{array}$ & $\begin{array}{l}1,100 \\
1,105 \\
1,105 \\
1,1000 \\
1,100\end{array}$ \\
\hline & & & & & $\begin{array}{l}12-12-80 \\
06-25-81 \\
08-08-85 \\
02-06-86 \\
05-08-86 \\
08-18-86 \\
11-04-86\end{array}$ & $\begin{array}{l}1,100 \\
1,100 \\
1,100 \\
1,110 \\
1,110 \\
1,105 \\
1,105\end{array}$ \\
\hline
\end{tabular}


Table 2.--Records of selected wells in Geauga, Lake, Ashtabula, Trumbull, and Portage Counties, Ohio--Continued

\begin{tabular}{|c|c|c|c|c|c|c|}
\hline $\begin{array}{l}\text { Wel } 1 \\
\text { numbe } r\end{array}$ & $\begin{array}{l}\text { Latitude } \\
\text { (degrees) }\end{array}$ & $\begin{array}{l}\text { Longitude } \\
\text { (degrees) }\end{array}$ & $\begin{array}{l}\text { Aqui fer } \\
\text { code }\end{array}$ & $\begin{array}{l}\text { Altitude } \\
\text { of land } \\
\text { surface } \\
\text { (feet) }\end{array}$ & $\begin{array}{c}\text { Date } \\
\text { water } \\
\text { level } \\
\text { measured }\end{array}$ & $\begin{array}{l}\text { Water- } \\
\text { level } \\
\text { altitude } \\
\text { (feet) }\end{array}$ \\
\hline \multirow[t]{2}{*}{ GE -119} & 412658 & 0810412 & 324 PSVL & 1,185 & $\begin{array}{l}08-20-80 \\
11-12-80 \\
08-15-85 \\
02-06-86 \\
04-30-86\end{array}$ & $\begin{array}{l}1,175 \\
1,175 \\
1,175 \\
1,175 \\
1,175\end{array}$ \\
\hline & & & & & $\begin{array}{l}08-19-86 \\
11-04-86\end{array}$ & $\begin{array}{l}1,175 \\
1,175\end{array}$ \\
\hline \multirow[t]{2}{*}{$G E-120$} & 413218 & 0811919 & 330BERE & 1,115 & $\begin{array}{l}06-17-80 \\
11-05-80 \\
08-07-85 \\
01-29-86 \\
04-18-86\end{array}$ & $\begin{array}{l}1,020 \\
1,020 \\
1,025 \\
1,015 \\
1,020\end{array}$ \\
\hline & & & & & $\begin{array}{l}08-22-86 \\
11-06-86\end{array}$ & $\begin{array}{l}1,020 \\
1,015\end{array}$ \\
\hline \multirow[t]{2}{*}{$\mathrm{GE}-121$} & 412746 & 0812020 & 330BERE & 1,085 & $\begin{array}{l}06-17-80 \\
11-05-80 \\
08-13-85 \\
01-31-86 \\
05-08-86\end{array}$ & $\begin{array}{l}1,015 \\
1,015 \\
1,010 \\
1,015 \\
1,010\end{array}$ \\
\hline & & & & & $\begin{array}{l}08-20-86 \\
11-06-86\end{array}$ & $\begin{array}{l}1,015 \\
1,015\end{array}$ \\
\hline \multirow[t]{2}{*}{$G E-122$} & 412410 & 0812239 & 330BERE & 1,010 & $\begin{array}{l}06-17-80 \\
11-06-80 \\
08-27-85 \\
02-04-86 \\
04-21-86\end{array}$ & $\begin{array}{l}950 \\
950 \\
950 \\
950 \\
950\end{array}$ \\
\hline & & & & & $\begin{array}{l}08-18-86 \\
11-03-86\end{array}$ & $\begin{array}{l}950 \\
950\end{array}$ \\
\hline $\mathrm{GE}-123$ & 412633 & 0811644 & 330BERE & 1,160 & $\begin{array}{l}06-17-80 \\
11-06-80 \\
08-14-85 \\
05-08-86 \\
08-20-86 \\
11-06-86\end{array}$ & $\begin{array}{l}1,060 \\
1,065 \\
1,060 \\
1,070 \\
1,070 \\
1,075\end{array}$ \\
\hline
\end{tabular}


Table 2.--Records of selected wel Is in Geauga, Lake, Ashtabula, Trumbull, and Portage Counties, Ohio--Continued

\begin{tabular}{|c|c|c|c|c|c|c|}
\hline $\begin{array}{l}\text { Well } 1 \\
\text { number }\end{array}$ & $\begin{array}{l}\text { Latitude } \\
\text { (degrees) }\end{array}$ & $\begin{array}{l}\text { Longitude } \\
\text { (degrees) }\end{array}$ & $\begin{array}{l}\text { Aqui fer } \\
\text { code }\end{array}$ & $\begin{array}{l}\text { Altitude } \\
\text { of land } \\
\text { surface } \\
\text { (feet) }\end{array}$ & $\begin{array}{c}\text { Date } \\
\text { water } \\
\text { level } \\
\text { measur eō }\end{array}$ & $\begin{array}{l}\text { Water- } \\
\text { level } \\
\text { altitude } \\
\text { (feet) }\end{array}$ \\
\hline \multirow[t]{2}{*}{$\mathrm{GE}-124$} & 413016 & 0811525 & 330BERE & 1,130 & $\begin{array}{l}06-17-80 \\
11-06-80 \\
08-07-85 \\
01-30-86 \\
05-09-86\end{array}$ & $\begin{array}{l}1,105 \\
1,105 \\
1,105 \\
1,105 \\
1,105\end{array}$ \\
\hline & & & & & $\begin{array}{l}08-21-86 \\
11-06-86\end{array}$ & $\begin{array}{l}1,105 \\
1,105\end{array}$ \\
\hline \multirow[t]{2}{*}{$\mathrm{GE}-125$} & 413100 & 0811055 & 330 BERE & 1,235 & $\begin{array}{l}06-17-80 \\
11-07-80 \\
12-12-80 \\
08-08-85 \\
02-07-86\end{array}$ & $\begin{array}{l}1,150 \\
1,165 \\
1,165 \\
1,155 \\
1,165\end{array}$ \\
\hline & & & & & $\begin{array}{l}05-09-86 \\
08-21-86 \\
11-04-86\end{array}$ & $\begin{array}{l}1,165 \\
1,145 \\
1,160\end{array}$ \\
\hline \multirow[t]{2}{*}{$\mathrm{GE}-126$} & 412212 & 0810253 & $330 \mathrm{BERE}$ & 1,070 & $\begin{array}{l}06-17-80 \\
11-06-80 \\
08-15-85 \\
02-05-86 \\
04-30-86\end{array}$ & $\begin{array}{l}955 \\
955 \\
980 \\
975 \\
980\end{array}$ \\
\hline & & & & & $\begin{array}{l}08-18-86 \\
11-04-86\end{array}$ & $\begin{array}{l}950 \\
955\end{array}$ \\
\hline $\mathrm{GE}-127$ & 412638 & 0810311 & $330 \mathrm{BERE}$ & 1,145 & $\begin{array}{l}08-28-85 \\
02-06-86 \\
05-07-86\end{array}$ & $\begin{array}{r}985 \\
1,030 \\
1,010\end{array}$ \\
\hline \multirow[t]{2}{*}{$\mathrm{GE}-130$} & 413623 & 0811010 & $330 \mathrm{BERE}$ & 1,215 & $\begin{array}{l}06-17-80 \\
11-14-80 \\
08-06-85 \\
02-07-86 \\
05-08-86\end{array}$ & $\begin{array}{l}1,130 \\
1,130 \\
1,130 \\
1,125 \\
1,125\end{array}$ \\
\hline & & & & & $\begin{array}{l}08-21-86 \\
11-05-86\end{array}$ & $\begin{array}{l}1,125 \\
1,130\end{array}$ \\
\hline
\end{tabular}


Table 2.--Recoras of selected wells in Geauga, Lake, Ashtabula, Trumbull, and Portage Counties, Ohio--Continued

\begin{tabular}{|c|c|c|c|c|c|c|}
\hline $\begin{array}{c}\text { Wel } 1 \\
\text { number }\end{array}$ & $\begin{array}{l}\text { Latitude } \\
\text { (degrees) }\end{array}$ & $\begin{array}{l}\text { Longitude } \\
\text { (degrees) }\end{array}$ & $\begin{array}{l}\text { Aqui fer } \\
\text { code }\end{array}$ & $\begin{array}{l}\text { Altitude } \\
\text { of land } \\
\text { surface } \\
\text { (feet) }\end{array}$ & $\begin{array}{c}\text { Date } \\
\text { water } \\
\text { leve1 } \\
\text { measured }\end{array}$ & $\begin{array}{l}\text { Water- } \\
\text { level } \\
\text { altitude } \\
\text { (feet) }\end{array}$ \\
\hline GE -135 & 412959 & 0810307 & $1120 \mathrm{TSH}$ & 1,110 & $\begin{array}{l}08-08-85 \\
02-06-86 \\
05-08-86 \\
08-19-86 \\
11-04-86\end{array}$ & $\begin{array}{l}1,095 \\
1,100 \\
1,100 \\
1,100 \\
1,100\end{array}$ \\
\hline$G E-136$ & 412841 & 0810232 & $330 \mathrm{CYHG}$ & 1,130 & $\begin{array}{l}08-08-85 \\
02-06-86 \\
05-08-86 \\
08-19-86 \\
11-04-86\end{array}$ & $\begin{array}{l}1,115 \\
1,115 \\
1,115 \\
1,115 \\
1,115\end{array}$ \\
\hline GE $-137 A$ & 413348 & 0810043 & $330 \mathrm{BERE}$ & 1.100 & $\begin{array}{l}08-08-85 \\
02-06-86 \\
05-08-86 \\
08-19-86\end{array}$ & $\begin{array}{l}1,065 \\
1,065 \\
1,065 \\
1,060\end{array}$ \\
\hline$G E-138$ & 412159 & 0811041 & 324 P SVL & 1,180 & $\begin{array}{l}08-14-85 \\
02-05-86 \\
05-07-86 \\
08-18-86 \\
11-03-86\end{array}$ & $\begin{array}{l}1,135 \\
1,135 \\
1,135 \\
1,135 \\
1,135\end{array}$ \\
\hline GE-139 & 412138 & 0810720 & 324 P SVL & 1,170 & $\begin{array}{l}08-15-85 \\
02-05-86 \\
05-07-86 \\
08-18-86 \\
11-03-86\end{array}$ & $\begin{array}{l}1,135 \\
1,135 \\
1,135 \\
1,135 \\
1,135\end{array}$ \\
\hline $\mathrm{GE}-140$ & 412318 & 0810737 & 324 PSVL & 1,220 & $\begin{array}{l}08-15-85 \\
02-05-86 \\
05-07-86 \\
08-18-86 \\
11-03-86\end{array}$ & $\begin{array}{l}1,175 \\
1,170 \\
1,170 \\
1,170 \\
1,170\end{array}$ \\
\hline $\mathrm{GE}-141$ & 412224 & 0810843 & $112 \mathrm{OTSH}$ & 1,120 & $\begin{array}{l}08-15-85 \\
02-05-86 \\
05-01-86 \\
08-18-86 \\
11-03-86\end{array}$ & $\begin{array}{l}1,110 \\
1,110 \\
1,110 \\
1,110 \\
1,110\end{array}$ \\
\hline
\end{tabular}


Table 2.--Records of selected wells in Geauga, Lake, Ashtabula, Trumbul 1 , and Portace counties, chio--Continued

\begin{tabular}{|c|c|c|c|c|c|c|}
\hline $\begin{array}{l}\text { Wel } 1 \\
\text { number }\end{array}$ & $\begin{array}{l}\text { Latitude } \\
\text { (degrees) }\end{array}$ & $\begin{array}{l}\text { Longitude } \\
\text { (degrees) }\end{array}$ & $\begin{array}{l}\text { Aquifer } \\
\text { cocie }\end{array}$ & $\begin{array}{l}\text { Altitude } \\
\text { of land } \\
\text { surface } \\
\text { (feet) }\end{array}$ & $\begin{array}{c}\text { Date } \\
\text { water } \\
\text { levej. } \\
\text { measureci }\end{array}$ & $\begin{array}{l}\text { Water- } \\
\text { level } \\
\text { altituc.e } \\
\text { (feet) }\end{array}$ \\
\hline$G E-143$ & 412529 & 0811320 & 324 PSVL & 1,230 & $\begin{array}{l}08-16-85 \\
02-04-86 \\
05-07-86 \\
08-18-86 \\
11-03-86\end{array}$ & $\begin{array}{l}1,220 \\
1,220 \\
1,220 \\
1,220 \\
1,215\end{array}$ \\
\hline $\mathrm{GE}-144$ & 412211 & 0811834 & 324 PSVL & 1,160 & $\begin{array}{l}08-28-85 \\
02-04-86 \\
05-07-86 \\
08-18-86 \\
11-03-86\end{array}$ & $\begin{array}{l}1,125 \\
1,125 \\
1,125 \\
1,125 \\
1,125\end{array}$ \\
\hline$G E-145$ & 413729 & 0810247 & 330 CYHG & 1,210 & $\begin{array}{l}09-18-85 \\
02-10-86 \\
05-08-86 \\
08-19-86 \\
11-05-86\end{array}$ & $\begin{array}{l}1,170 \\
1,160 \\
1,160 \\
1,160 \\
1,160\end{array}$ \\
\hline $\mathrm{GE}-147$ & 412845 & 0810301 & 330 С YHG & 1,120 & $\begin{array}{l}09-18-85 \\
02-06-86 \\
04-30-86 \\
08-18-86 \\
11-04-86\end{array}$ & $\begin{array}{l}1,115 \\
1,120 \\
1,120 \\
1,120 \\
1,120\end{array}$ \\
\hline$G E-150$ & 413155 & 0812149 & 324 PSVI & 1,220 & $\begin{array}{l}02-13-86 \\
05-09-86 \\
08-22-86 \\
11-06-86\end{array}$ & $\begin{array}{l}1,195 \\
1,195 \\
1,195 \\
1,195\end{array}$ \\
\hline GE-151 & 412319 & 0811350 & 324 PSVL & 1,270 & $\begin{array}{l}09-19-85 \\
02-04-86 \\
04-29-86 \\
08-18-86 \\
11-03-86\end{array}$ & $\begin{array}{l}1,185 \\
1,185 \\
1,185 \\
1,185 \\
1,185\end{array}$ \\
\hline GE-152 & 413246 & 0811440 & 324 P SVL & 1,300 & $\begin{array}{l}09-17-85 \\
01-30-86 \\
05-09-86 \\
08-21-86 \\
11-06-86\end{array}$ & $\begin{array}{l}1,265 \\
1,270 \\
1,270 \\
1,270 \\
1,270\end{array}$ \\
\hline
\end{tabular}


Table 2.--Records of selecteā wells in Geauga, Lake, Ashtabula, Trumbul1, and Portage Counties, Ohio--Continued

\begin{tabular}{|c|c|c|c|c|c|c|}
\hline $\begin{array}{c}\text { Wel } 1 \\
\text { number }\end{array}$ & $\begin{array}{l}\text { Latitude } \\
\text { (ciegrees) }\end{array}$ & $\begin{array}{c}\text { Longitude } \\
\text { (degrees) }\end{array}$ & $\begin{array}{l}\text { Aquifer } \\
\text { code }\end{array}$ & $\begin{array}{l}\text { Altitude } \\
\text { of land } \\
\text { surface } \\
\text { (feet) }\end{array}$ & $\begin{array}{c}\text { Date } \\
\text { water } \\
\text { level } \\
\text { meas ur ed }\end{array}$ & $\begin{array}{l}\text { Water- } \\
\text { level } \\
\text { altitude } \\
\text { (feet) }\end{array}$ \\
\hline GE- 153 & 413415 & 0811609 & $1120 \mathrm{TSH}$ & 1,275 & $\begin{array}{l}09-17-85 \\
01-29-86 \\
05-09-86 \\
08-21-86 \\
11-05-86\end{array}$ & $\begin{array}{l}1,210 \\
1,210 \\
1,210 \\
1,215 \\
1,210\end{array}$ \\
\hline$G E-156$ & 412835 & 0811858 & 324 PSVL & 1,210 & $\begin{array}{l}02-13-86 \\
05-08-86 \\
08-20-86 \\
11-06-86\end{array}$ & $\begin{array}{l}1,150 \\
1,150 \\
1,150 \\
1,150\end{array}$ \\
\hline GE -157 & 413628 & 0810605 & $1120 \mathrm{TSH}$ & 1,185 & $\begin{array}{l}09-19-85 \\
02-07-86 \\
05-08-86 \\
08-21-86 \\
11-05-86\end{array}$ & $\begin{array}{l}1,180 \\
1,180 \\
1,180 \\
1,180 \\
1,180\end{array}$ \\
\hline GE -159 & 412420 & 0811021 & 330BERE & 1,140 & $\begin{array}{l}02-05-86 \\
04-30-86 \\
08-18-86 \\
11-03-86\end{array}$ & $\begin{array}{l}1,125 \\
1,120 \\
1,120 \\
1,120\end{array}$ \\
\hline $\mathrm{GE}-160$ & 412520 & 0810845 & $330 \mathrm{CYHG}$ & 1,135 & $\begin{array}{l}02-05-86 \\
05-07-86\end{array}$ & $\begin{array}{l}1,100 \\
1,125\end{array}$ \\
\hline $\mathrm{GE}-161$ & 412304 & 0811023 & 324 PSVL & 1,180 & $\begin{array}{l}02-05-86 \\
05-07-86 \\
08-18-86 \\
11-03-86\end{array}$ & $\begin{array}{l}1,160 \\
1,160 \\
1,155 \\
1,155\end{array}$ \\
\hline$G E-162$ & 412511 & 0810328 & 324 PSVL & 1,210 & $\begin{array}{l}02-05-86 \\
04-30-86 \\
08-18-86 \\
11-04-86\end{array}$ & $\begin{array}{l}1,170 \\
1,170 \\
1,170 \\
1,170\end{array}$ \\
\hline GE -163 & 412415 & 0810335 & 324 PSVL & 1,180 & $\begin{array}{l}02-05-86 \\
05-08-86 \\
08-18-86 \\
11-04-86\end{array}$ & $\begin{array}{l}1,175 \\
1,170 \\
1,170 \\
1,170\end{array}$ \\
\hline GE -165 & 412319 & 0811630 & $1120 \mathrm{TSH}$ & 1,165 & $\begin{array}{l}02-04-86 \\
05-01-86 \\
08-18-86 \\
11-03-86\end{array}$ & $\begin{array}{l}1,155 \\
1,155 \\
1,155 \\
1,155\end{array}$ \\
\hline
\end{tabular}


Table 2.--Records of selected wells in Geauga, Lake, Ashtabula, Trumbull, and Portage Counties, Ohio--Continued

\begin{tabular}{|c|c|c|c|c|c|c|}
\hline $\begin{array}{l}\text { Wel1 } \\
\text { number }\end{array}$ & $\begin{array}{l}\text { Latitude } \\
\text { (degrees) }\end{array}$ & $\begin{array}{l}\text { Longitude } \\
\text { (degrees) }\end{array}$ & $\begin{array}{l}\text { Aquifer } \\
\text { code }\end{array}$ & $\begin{array}{l}\text { Altitude } \\
\text { of land } \\
\text { surface } \\
\text { (feet) }\end{array}$ & $\begin{array}{c}\text { Date } \\
\text { water } \\
\text { level } \\
\text { measured }\end{array}$ & $\begin{array}{l}\text { Water- } \\
\text { level } \\
\text { altitude } \\
\text { (feet) }\end{array}$ \\
\hline$G E-166$ & 412451 & 0811624 & 324 PSVL & 1,260 & $\begin{array}{l}02-04-86 \\
05-07-86 \\
08-18-86 \\
11-03-86\end{array}$ & $\begin{array}{l}1,210 \\
1,210 \\
1,205 \\
1,205\end{array}$ \\
\hline GE-1 69 & 412628 & 0811228 & $330 \mathrm{CYHG}$ & 1,115 & $\begin{array}{l}01-30-86 \\
04-29-86 \\
08-20-86 \\
11-06-86\end{array}$ & $\begin{array}{l}1,115 \\
1,115 \\
1,115 \\
1,115\end{array}$ \\
\hline$G E-170$ & 412310 & 0812130 & 330 CYHG & 1,110 & $\begin{array}{l}02-04-86 \\
05-07-86 \\
08-18-86 \\
11-03-86\end{array}$ & $\begin{array}{l}1,060 \\
1,060 \\
1,060 \\
1,065\end{array}$ \\
\hline $\mathrm{GE}-171$ & 412511 & 0812259 & 33 OCYHG & 985 & $\begin{array}{l}02-04-86 \\
05-07-86 \\
08-18-86 \\
11-03-86\end{array}$ & $\begin{array}{l}930 \\
930 \\
930 \\
930\end{array}$ \\
\hline $\mathrm{GE}-172$ & 413409 & 0811551 & 330 CYHG & 1,270 & $\begin{array}{l}02-13-86 \\
05-09-86 \\
08-21-86 \\
11-05-86\end{array}$ & $\begin{array}{l}1,220 \\
1,220 \\
1,215 \\
1,215\end{array}$ \\
\hline $\mathrm{GE}-173$ & 412142 & 0812123 & 324 PSV & 1,095 & $\begin{array}{l}02-04-86 \\
08-18-86 \\
11-03-86\end{array}$ & $\begin{array}{l}1,090 \\
1,090 \\
1,090\end{array}$ \\
\hline $\mathrm{GE}-174$ & 412907 & 0812021 & $1120 \mathrm{TSH}$ & 1,130 & $\begin{array}{l}01-31-86 \\
04-23-86 \\
08-20-86 \\
11-06-86\end{array}$ & $\begin{array}{l}1,100 \\
1,095 \\
1,100 \\
1,100\end{array}$ \\
\hline $\mathrm{GE}-175$ & 412841 & 0812149 & $1120 \mathrm{TSH}$ & 1,045 & $\begin{array}{l}01-31-86 \\
04-23-86 \\
08-20-86 \\
11-06-86\end{array}$ & $\begin{array}{l}1,015 \\
1,015 \\
1,010 \\
1,015\end{array}$ \\
\hline $\mathrm{GE}-176$ & 413521 & 0811431 & 324 P SVL & 1,310 & $\begin{array}{l}02-13-86 \\
05-08-86 \\
08-21-86 \\
11-05-86\end{array}$ & $\begin{array}{l}1,265 \\
1,265 \\
1,260 \\
1,265\end{array}$ \\
\hline
\end{tabular}


Table 2.--Recorås of selected wells in Geauga, Lake, Ashtabula, Trumbull, and Portage Counties, Ohio--Continued

\begin{tabular}{|c|c|c|c|c|c|c|}
\hline $\begin{array}{c}\text { Wel } 1 \\
\text { number }\end{array}$ & $\begin{array}{l}\text { Latitude } \\
\text { (degrees) }\end{array}$ & $\begin{array}{l}\text { Longitude } \\
\text { (degrees) }\end{array}$ & $\begin{array}{l}\text { Aquifer } \\
\text { code }\end{array}$ & $\begin{array}{l}\text { Altitude } \\
\text { of land } \\
\text { surface } \\
\text { (feet) }\end{array}$ & $\begin{array}{c}\text { Date } \\
\text { water } \\
\text { level } \\
\text { measured }\end{array}$ & $\begin{array}{l}\text { Water- } \\
\text { level } \\
\text { altitude } \\
\text { (feet) }\end{array}$ \\
\hline GE -177 & 413408 & 0810830 & 1120TSH & 1,190 & $\begin{array}{l}02-06-86 \\
05-09-86 \\
08-21-86 \\
11-04-86\end{array}$ & $\begin{array}{l}1,185 \\
1,185 \\
1,170 \\
1,180\end{array}$ \\
\hline $\mathrm{GE}-178$ & 413138 & 0810842 & 324 PSVL & 1,310 & $\begin{array}{l}02-06-86 \\
05-09-86 \\
08-21-86 \\
11-04-86\end{array}$ & $\begin{array}{l}1,255 \\
1,255 \\
1,255 \\
1,255\end{array}$ \\
\hline GE-179 & 413410 & 0812142 & 330BERE & 1,070 & $\begin{array}{l}02-13-86 \\
05-08-86 \\
08-22-86 \\
11-06-86\end{array}$ & $\begin{array}{l}1,010 \\
1,010 \\
1,010 \\
1,010\end{array}$ \\
\hline $\mathrm{GE}-180$ & 413114 & 0812016 & 324 P SVL & 1,210 & $\begin{array}{l}01-29-86 \\
04-23-86 \\
05-09-86 \\
08-29-86 \\
11-06-86\end{array}$ & $\begin{array}{l}1,180 \\
1,180 \\
1,180 \\
1,180 \\
1,180\end{array}$ \\
\hline GE-181 & 413118 & 0811936 & 324 PSVL & 1,230 & $\begin{array}{l}01-29-86 \\
05-08-86 \\
08-20-86 \\
11-06-86\end{array}$ & $\begin{array}{l}1,220 \\
1,220 \\
1,220 \\
1,220\end{array}$ \\
\hline $\mathrm{GE}-182$ & 413223 & 0812019 & $330 \mathrm{CYHG}$ & 1,180 & $01-28-86$ & 1,125 \\
\hline GE -183 & 412429 & 0810451 & 324 PSVL & 1,220 & $\begin{array}{l}02-05-86 \\
05-08-86 \\
08-18-86 \\
11-04-86\end{array}$ & $\begin{array}{l}1,180 \\
1,180 \\
1,180 \\
1,180\end{array}$ \\
\hline GE- 184 & 413020 & 0811853 & $1120 \mathrm{TSH}$ & 1,120 & $\begin{array}{l}01-29-86 \\
05-08-86 \\
08-22-86 \\
11-06-86\end{array}$ & $\begin{array}{l}1,045 \\
1,045 \\
1,045 \\
1,045\end{array}$ \\
\hline $\mathrm{GE}-185$ & 413630 & 0811450 & $330 \mathrm{CYHG}$ & 1,260 & $\begin{array}{l}02-13-86 \\
05-08-86 \\
08-21-86 \\
11-05-86\end{array}$ & $\begin{array}{l}1,230 \\
1,230 \\
1,220 \\
1,225\end{array}$ \\
\hline
\end{tabular}


Table 2.--Records of selected wells in Geauga, Lake, Ashtabula, Trumbull, and Portace Counties, Ohio--Continued

\begin{tabular}{|c|c|c|c|c|c|c|}
\hline $\begin{array}{l}\text { Wel1 } 1 \\
\text { number }\end{array}$ & $\begin{array}{l}\text { Latitude } \\
\text { (degrees) }\end{array}$ & $\begin{array}{l}\text { Longitude } \\
\text { (ciegrees) }\end{array}$ & $\begin{array}{l}\text { Aquifer } \\
\text { cocie }\end{array}$ & $\begin{array}{l}\text { Altitude } \\
\text { of land } \\
\text { surface } \\
\text { (feet) }\end{array}$ & $\begin{array}{c}\text { Date } \\
\text { water } \\
\text { level. } \\
\text { measur eci }\end{array}$ & $\begin{array}{l}\text { Water- } \\
\text { level } \\
\text { altituce } \\
\text { (feet) }\end{array}$ \\
\hline $\mathrm{GE}-186$ & 413647 & 0811200 & 330 CYHG & $1,1.50$ & $\begin{array}{l}01-29-86 \\
04-15-86 \\
08-21-86 \\
11-05-86\end{array}$ & $\begin{array}{l}1,115 \\
1,105 \\
1,105 \\
1,105\end{array}$ \\
\hline $\mathrm{GE}-190$ & 413734 & 0811521 & $1120 \mathrm{TSH}$ & 1,070 & $\begin{array}{l}01-29-86 \\
05-08-86\end{array}$ & $\begin{array}{l}1,055 \\
1,050\end{array}$ \\
\hline$G E-193$ & 413506 & 0811618 & 324 PSVL & 1,300 & $\begin{array}{l}01-30-86 \\
05-09-86 \\
08-21-86 \\
11-05-86\end{array}$ & $\begin{array}{l}1,250 \\
1,250 \\
1,250 \\
1,245\end{array}$ \\
\hline GE- 195 & 413513 & 0811107 & $1120 T S H$ & 1,130 & $\begin{array}{l}02-10-86 \\
05-08-86 \\
08-21-86 \\
11-05-86\end{array}$ & $\begin{array}{l}1,115 \\
1,115 \\
1,115 \\
1,115\end{array}$ \\
\hline GE-196 & 413808 & 0810347 & 324 PSVL & 1,300 & $\begin{array}{l}02-10-86 \\
05-08-86 \\
08-19-86 \\
11-05-86\end{array}$ & $\begin{array}{l}1,235 \\
1,235 \\
1,235 \\
1,235\end{array}$ \\
\hline$G E-197$ & 413957 & 0810118 & $330 \mathrm{BERE}$ & 1,135 & $\begin{array}{l}02-11-86 \\
05-08-86 \\
08-19-86 \\
11-05-86\end{array}$ & $\begin{array}{l}1,090 \\
1,070 \\
1,070 \\
1,070\end{array}$ \\
\hline $\mathrm{GE}-198$ & 414058 & 0810100 & 330BERE & 1.070 & $\begin{array}{l}02-11-86 \\
05-08-86 \\
08-19-86 \\
11-05-86\end{array}$ & $\begin{array}{l}1,055 \\
1,055 \\
1,055 \\
1,055\end{array}$ \\
\hline $\mathrm{GE}-199$ & 414106 & 0810414 & 324 PSVL & 1.255 & $\begin{array}{l}02-11-86 \\
05-08-86 \\
08-19-86 \\
11-05-86\end{array}$ & $\begin{array}{l}1,245 \\
1,240 \\
1,240 \\
1,240\end{array}$ \\
\hline$G E-202$ & 413607 & 0810325 & 324 PSVI & 1,245 & $\begin{array}{l}02-10-86 \\
04-16-86 \\
08-19-86 \\
11-05-86\end{array}$ & $\begin{array}{l}1,220 \\
1,220 \\
1,220 \\
1,220\end{array}$ \\
\hline
\end{tabular}


T'able 2.--Recorás of selectea wells in Geauga, Lake, Ashtabula, Trumbull, and Eortage Counties, Ohio--Continued

\begin{tabular}{|c|c|c|c|c|c|c|}
\hline $\begin{array}{c}\text { Wel I } \\
\text { number }\end{array}$ & $\begin{array}{l}\text { Latitude } \\
\text { (cegrees) }\end{array}$ & $\begin{array}{l}\text { Longitude } \\
\text { (degrees) }\end{array}$ & $\begin{array}{l}\text { Aquifer } \\
\text { code }\end{array}$ & $\begin{array}{l}\text { Altitude } \\
\text { of land } \\
\text { surface } \\
\text { (feet) }\end{array}$ & $\begin{array}{c}\text { Date } \\
\text { water } \\
\text { level } \\
\text { measureả }\end{array}$ & $\begin{array}{l}\text { Water- } \\
\text { level } \\
\text { altitude } \\
\text { (feet) }\end{array}$ \\
\hline $\mathrm{GE}-204$ & 413256 & 0810458 & 324 PSVL & 1,230 & $\begin{array}{l}02-06-86 \\
05-08-86 \\
08-19-86 \\
11-04-86\end{array}$ & $\begin{array}{l}1,220 \\
1,220 \\
1,220 \\
1,220\end{array}$ \\
\hline$L-86$ & 414008 & 0810644 & 330 BERE & 1,080 & $02-11-86$ & 1,060 \\
\hline$L-88$ & 413841 & 0810850 & $1120 \mathrm{TSH}$ & 1,145 & $02-11-86$ & 1,075 \\
\hline$L-89$ & 413830 & 0811353 & 330 BERE & 1,070 & $02-14-86$ & 1,060 \\
\hline$L-90$ & 413434 & 0811813 & $112 \mathrm{OTSH}$ & 925 & $02-13-86$ & 905 \\
\hline$L-91$ & 413501 & 0812055 & $1120 \mathrm{TSH}$ & 1,065 & $02-13-86$ & 1,045 \\
\hline$A B-125$ & 413206 & 0805959 & $330 \mathrm{CYHG}$ & 1,085 & $02-11-86$ & 1,070 \\
\hline$A B-126$ & 413646 & 0805931 & 330 BERE & 1,080 & $02-11-86$ & 1,065 \\
\hline$A B-127$ & 413944 & 0805926 & 330BERE & 1,060 & $02-11-86$ & 1,050 \\
\hline$T-5$ & 412753 & 0805831 & 330 BERE & 965 & $02-11-86$ & 930 \\
\hline$T-6$ & 412333 & 0805824 & 330BERE & 885 & $02-11-86$ & 870 \\
\hline $\mathrm{PO}-7$ & 411950 & 0810227 & 330 CYHG & 970 & $02-12-86$ & 955 \\
\hline $\mathrm{PO}-8$ & 411939 & 0810300 & 324 PSVL & 1,130 & $02-11-56$ & 1,095 \\
\hline $\mathrm{PO}-9$ & 411840 & 0810541 & 330 CYHG & 1,050 & $02-12-86$ & 1,030 \\
\hline $\mathrm{PO}-10$ & 411858 & 0810540 & 324 PSVL & 1,100 & $02-12-86$ & 1,075 \\
\hline $\mathrm{PO}-11$ & 411713 & 0810823 & 324 PSVL & 1,100 & $02-12-86$ & 1,045 \\
\hline $\mathrm{PO}-12$ & 411743 & 0810941 & 324 PSVL & 1,310 & $02-12-86$ & 1,270 \\
\hline $\mathrm{PO}-13$ & 411958 & 0810815 & 330 CYHG & 1,100 & $02-12-86$ & 1,085 \\
\hline $\mathrm{PO}-14$ & 411915 & 0811319 & 324 PSVL & 1,210 & $02-12-86$ & 1,200 \\
\hline $\mathrm{PO}-15$ & 411839 & 0811656 & 324 PSVL & 1,170 & $02-12-86$ & 1,135 \\
\hline
\end{tabular}


Table 2.--Records of selected wells in Geauga, Lake, Ashtabula, Trumbul1, and Portage Counties, Ohio--Continued

\begin{tabular}{lllllll}
\hline $\begin{array}{c}\text { Wel1 } \\
\text { number }\end{array}$ & $\begin{array}{c}\text { Latitude } \\
\text { (degrees) }\end{array}$ & $\begin{array}{c}\text { Longitude } \\
\text { (degrees) }\end{array}$ & $\begin{array}{c}\text { Altitude } \\
\text { of land } \\
\text { sode } \\
\text { curface } \\
\text { (feet) }\end{array}$ & $\begin{array}{c}\text { Date } \\
\text { water } \\
\text { level } \\
\text { measured }\end{array}$ & $\begin{array}{c}\text { Water- } \\
\text { level } \\
\text { altitude } \\
\text { (fet) }\end{array}$ \\
\hline PO-16 & 411524 & 0811655 & $1120 T S H$ & 1,110 & $02-12-86$ & 1,090 \\
PO-18 & 411904 & 0811835 & 1120TSH & 1,135 & $02-12-86$ & 1,120 \\
\hline
\end{tabular}

